\title{
WestVirginiaUniversity
}

THE RESEARCH REPOSITORY @ WVU

Graduate Theses, Dissertations, and Problem Reports

2007

\section{Automated ventricular measurements using Gabor wavelets}

Hemalatha Sampath

West Virginia University

Follow this and additional works at: https://researchrepository.wvu.edu/etd

\section{Recommended Citation}

Sampath, Hemalatha, "Automated ventricular measurements using Gabor wavelets" (2007). Graduate Theses, Dissertations, and Problem Reports. 4333.

https://researchrepository.wvu.edu/etd/4333

This Thesis is protected by copyright and/or related rights. It has been brought to you by the The Research Repository @ WVU with permission from the rights-holder(s). You are free to use this Thesis in any way that is permitted by the copyright and related rights legislation that applies to your use. For other uses you must obtain permission from the rights-holder(s) directly, unless additional rights are indicated by a Creative Commons license in the record and/ or on the work itself. This Thesis has been accepted for inclusion in WVU Graduate Theses, Dissertations, and Problem Reports collection by an authorized administrator of The Research Repository @ WVU. For more information, please contact researchrepository@mail.wvu.edu. 
Automated Ventricular Measurements using Gabor Wavelets

Hemalatha Sampath

Thesis submitted to the

College of Engineering and Mineral Resources

at West Virginia University

in partial fulfillment of the requirements

for the degree of

\author{
Master of Science \\ in \\ Electrical Engineering
}

Dr. Donald A. Adjeroh, Ph.D., Chair

Dr. Susan K. Lemieux, Ph.D., Co-Chair

Dr. Aina Puce, Ph.D.,

Dr. Tim McGraw, Ph. D.,

Dr. Arun A. Ross, Ph.D.

Lane Department of Computer Science and Electrical Engineering

Center for Advanced Imaging, WVU School of Medicine

Morgantown, West Virginia

2007

Keywords:

MRI, Image Segmentation, Gabor wavelets, Lateral ventricle, Third ventricle, Texture based segmentation 


\section{ABSTRACT \\ Automated Ventricular Measurements using Gabor Wavelets}

Hemalatha Sampath

Magnetic Resonance Imaging (MRI) is one of the widely used medical technologies for diagnosis of various degenerative diseases like Alzheimer's disease. In degenerative diseases the brain atrophies resulting in enlarged ventricles. The segmentation of the ventricle and the total ventricle volume measurement can be important in the analysis of the neurological disease severity and in studies of animal models of disease used to advance treatments for people. This thesis is focused on using Gabor wavelets for ventricular measurements in a rabbit model of Alzheimer's disease.

Standard intensity-based tools like Statistical Parametric Mapping (SPM) used for human brain segmentation requires a priori knowledge of the data. As there is no available rabbit brain atlas incorporated into the algorithm, SPM failed for rabbit brain segmentation. Hence, texture-based segmentation was used to solve the problem. Gabor wavelet decomposition of the textures in an image provides a powerful mathematical tool for feature extraction. The features contain important frequency information that can be estimated from biological parameters to choose the wavelet set. Our goal is to develop an automated method for extracting ventricular structures from whole-head rabbit MRI.

Rabbit brain segmentation is a complex process in low contrast MRI images. Hence the Gabor wavelets were first studied on the high resolution digital histological rabbit brain images. With the success of ventricle segmentation in the histology images, the Gabor parameters were modified for the rabbit brain MR images. Initially, manually guided segmentation results were obtained using Gabor wavelets. This work continued further to automate the process of brain region of interest (ROI) selection to automate the ventricle extraction process. 


\section{DEDICATIONS}

To the Holy Mother

To my grandfather

To my parents

To my sister

To my uncle and aunt

To my professor

To all my friends 


\section{ACKNOWLEDGEMENTS}

There are not enough words to explain the most exciting part of my life during my masters with the blessings of the Holy Mother. The completion of my master's career would not have been possible without the support and encouragement of many people. I reflect back on the last two and a half years and on the individuals who have left an impact on me, I write this very important and difficult section.

I am highly obliged to my advisor Dr. Susan Lemieux for her motivation, support, guidance and encouragement during the entire period of this research work. I am very fortunate to be her student and for having an opportunity to work with her. I am honored to have Dr. Donald Adjeroh as my advisor and committee chair. I thank you for all the special guidance to complete this work. The last one year when I worked with him, I gained so much knowledge that I can say it got the best out of me.

My deep respect and special thanks to Dr. Aina Puce for her valuable time and assistance during my research work. Her comments were very helpful in enhancing the thesis presentation. I would like to extend my gratitude to Dr. Tim McGraw and Dr. Arun Ross, my committee members, for their patience, and valuable suggestions.

Special thanks to Dr. Bernard Schreurs for providing me an opportunity to be a part of his research work. I would also like to thank Dr. Lauren Burhans for the providing me the data which was the foundation for this work. I would like to thank the members of Center for Advanced Imaging, Department of Radiology for all their help and guidance. The data for this study was collected with the support of NIH grant NIH-AG 232011.

I am very fortunate for the life and guidance that my parents have given me. My heartfelt gratitude to my sister Shrilatha for her prayers and support. My profound thanks to my uncle Prasad and aunt Sheela. Their constant encouragement and motivation helped me to make it all the way to US for MS. I would have never got this far without you all. I owe everything that I am to all of you. My earnest thanks to my fathers friends and my other friends in India for all their wishes and support.

Senthil, without you I would not even have thought of MS. I am very thankful to you and your parents. I am very grateful to Niveditha, who helped in getting admission to WVU and taking care of me very well.

Last but not the least, thanks to Sukanya, Arvind, Nitin, Abishek, Avinash, Balaji, Saideepti, Deepika, Mahitha and Deepthi to be with me and I would never forget all the fun I had with all of them in Morgantown. 


\section{TABLE OF CONTENTS}

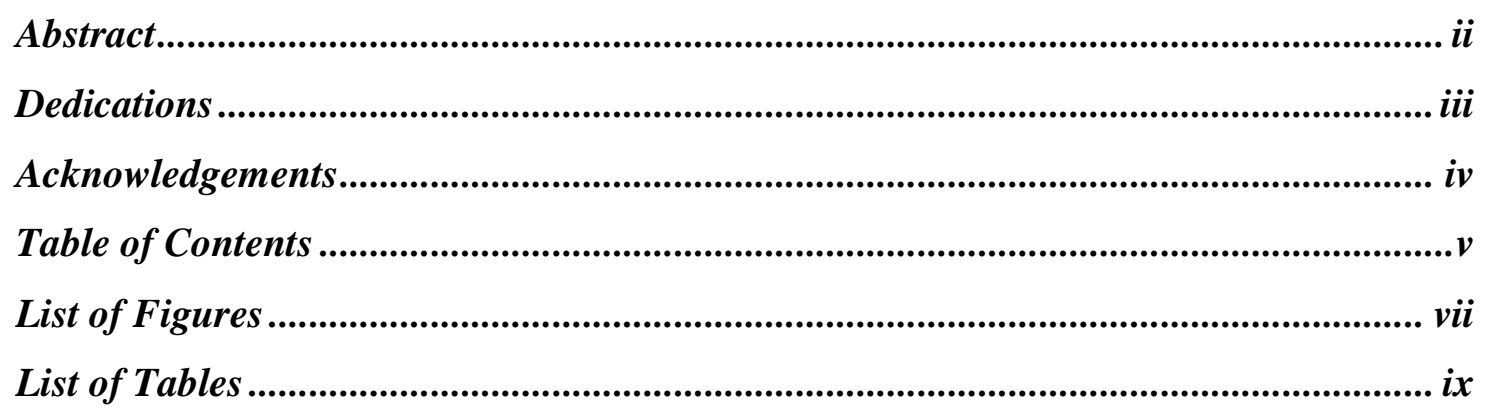

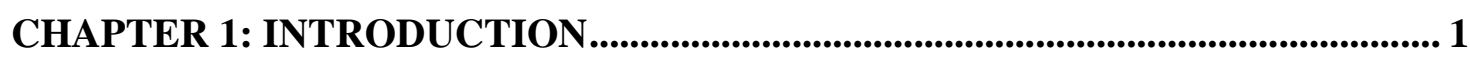

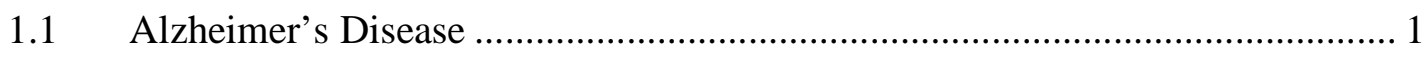

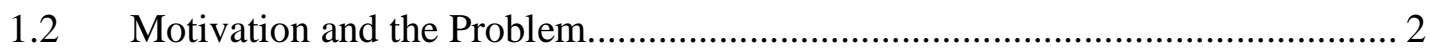

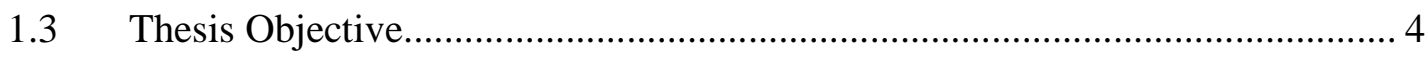

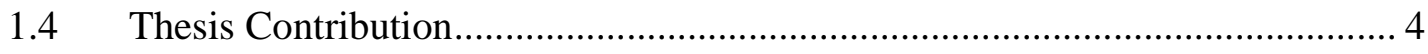

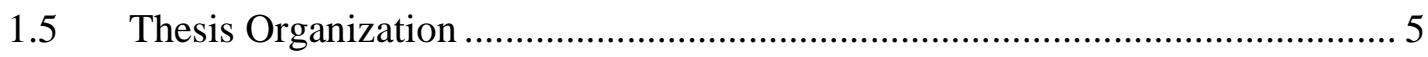

CHAPTER 2: BACKGROUND AND LITERATURE REVIEW .............................. 6

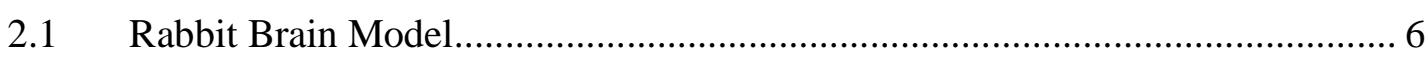

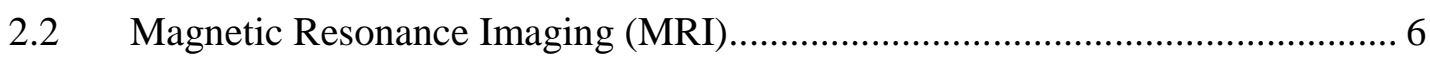

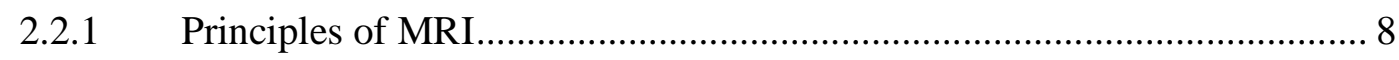

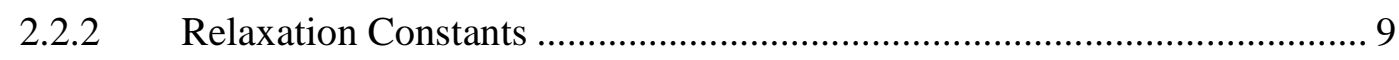

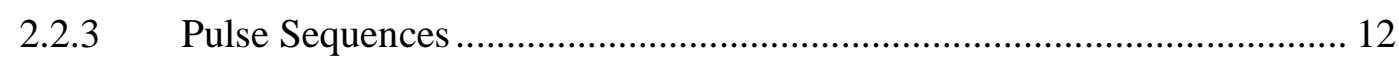

2.3 Introduction to Texture-Based Segmentation ............................................. 18

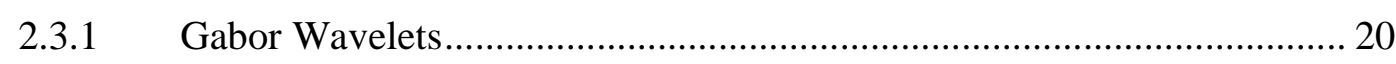

2.4 Gabor Wavelets in Medical Imaging ........................................................ 22

2.5 Gabor Wavelets in the analysis of Rabbit Brain Images ................................ 23

2.6 Ventricular Measurements and Analysis ..................................................... 25

2.7 Other Related Work - Classification and Segmentation ................................ 26

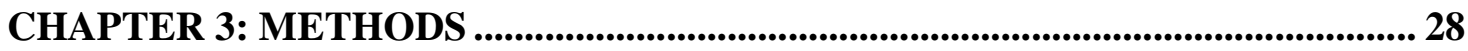

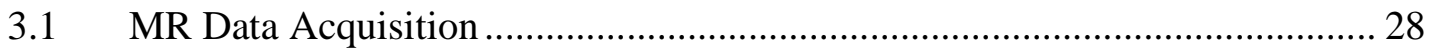




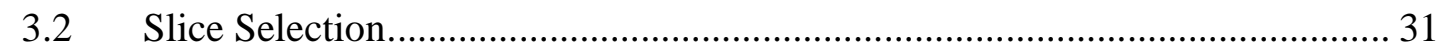

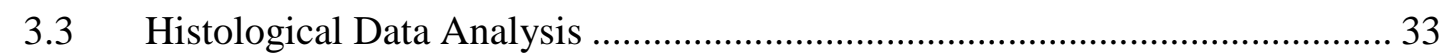

3.4 Stage 1: Performance Test of Gabor Wavelets on Rabbit Brain MRI.............. 34

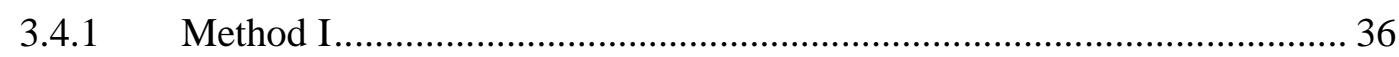

3.4.2 Method II (With adjustment of image intensity range) .......................... 37

3.5 Stage 2: Gabor Wavelets and Histological Data............................................. 38

3.6 Stage 3: Gabor Wavelets of Rabbit Brain MRI ............................................... 41

3.6.1 Manually Guided Segmentation Process ............................................... 41

3.6.2 Automated Ventricle Segmentation Process............................................ 42

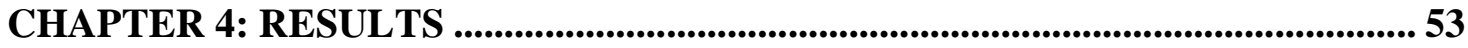

4.1 Stage 1: Performance Test of Gabor Wavelets on Rabbit Brain MRI.............. 53

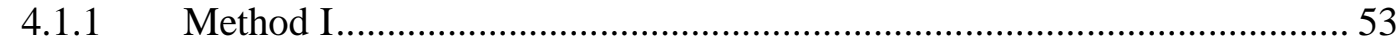

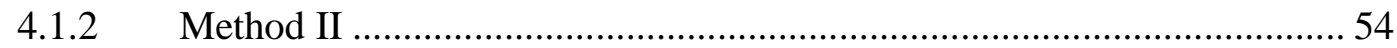

4.2 Stage 2: Gabor Wavelets on Histological Images........................................... 55

4.3 Stage 3: Gabor wavelets on Rabbit Brain MRI .............................................. 59

4.3.1 Manually Guided Segmentation Process .................................................. 59

4.3.2 Automated Ventricle Segmentation Process ............................................. 62

CHAPTER 5: DISCUSSION AND CONCLUSIONS ........................................................ 68

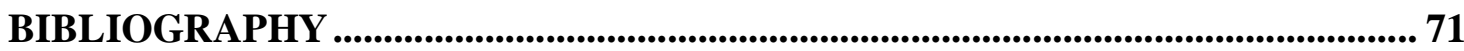




\section{LIST OF FIGURES}

Figure 1.1: Comparison of rabbit brain against human brain [64] ................................ 3

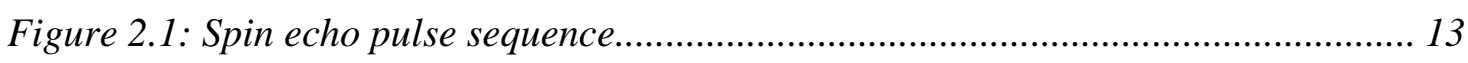

Figure 2.2: Fast spin echo imaging ............................................................................ 14

Figure 2.3: Gradient recalled echo pulse sequence ...................................................... 15

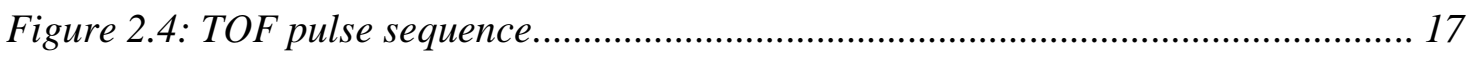

Figure 2.5: Different textures from Broadatz album [65]........................................... 19

Figure 2.6: Gabor wavelets for 4 scales and 6 orientations ........................................... 21

Figure 2.7: Comparison of the nissl-stained images and facial images [37] .................. 24

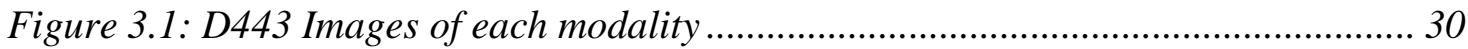

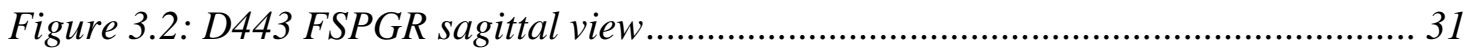

Figure 3.3: Alternate slices of D443 FSPGR Images..................................................... 32

Figure 3.4: View of coronal digital histology image ........................................................ 33

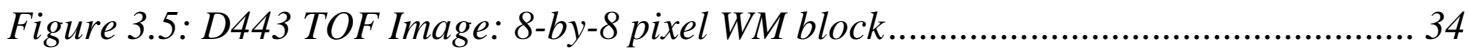

Figure 3.6: D443 TOF Image: 8-by-8 pixel GM block ....................................................... 34

Figure 3.7: Ventricle width measurements .................................................................... 35

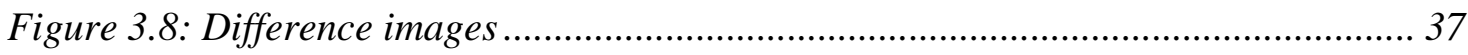

Figure 3.9: Flowchart for ventricle extraction process in histological images ............... 39

Figure 3.10: Gabor filtering and thresholding process............................................... 40

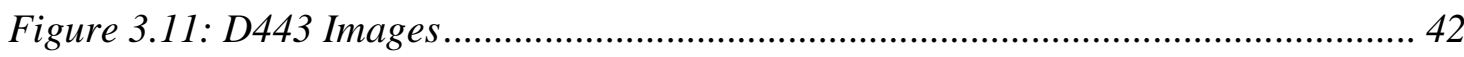

Figure 3.12: Flowchart of automated ventricle extraction process from whole head rabbit

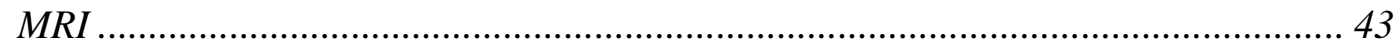

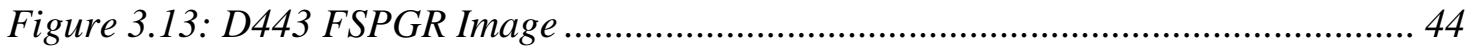

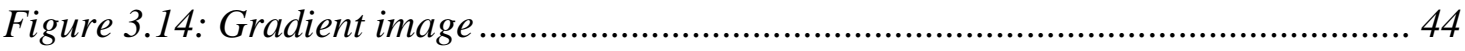

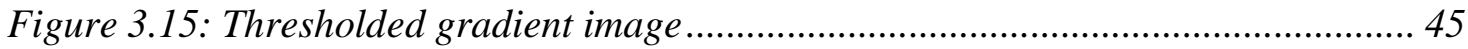

Figure 3.16: Reduced whole head rabbit MR image for peak detection ......................... 46

Figure 3.17: Reduced brain region after peak detection .................................................. 47

Figure 3.18: Binary image to measure ventricles width................................................. 47

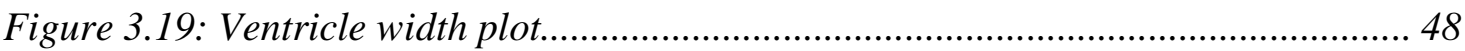


Figure 3.20: Imregionalmax function plot identifying peaks 49

Figure 3.21: Automated seed point region selection .......................................................... 50

Figure 3.22: Automated selection of brain ROI with ventricles...................................... 51

Figure 4.1: Euclidean distance plot versus modalities.................................................. 53

Figure 4.2: Euclidean distance plot versus difference images ...................................... 54

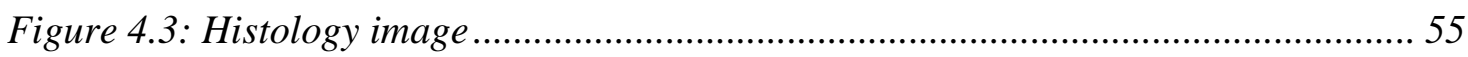

Figure 4.4: Brain ROI with ventricles and seed point selection (red)............................. 55

Figure 4.5: Ventricles overlaid on Histology Image …................................................. 57

Figure 4.6: Ventricles overlaid on Histology Image …..................................................... 58

Figure 4.7: Manual seed point selection - D443 FSPGR Image 24............................... 60

Figure 4.8: Automated seed point selection - D443 FSPGR Image 24 .......................... 60

Figure 4.9: Manual seed point selection - D443 TOF Image 27.................................. 61

Figure 4.10: Automated seed point selection - D443 TOF Image 27............................ 61

Figure 4.11: D443 FSPGR Image 25 - Automated ventricle extraction process ............. 62

Figure 4.12: D443 FSPGR Image 20 - Automated ventricle extraction process ............. 63

Figure 4.13: D443 FSPGR Image 23 - Automated ventricle extraction process ............. 64

Figure 4.14: D443 FSPGR Image 29 - Automated ventricle extraction process ............. 65

Figure 4.15: D443 FSPGR Image 35 - Automated ventricle extraction process ............. 66

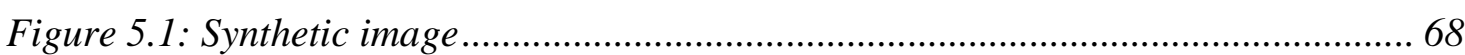

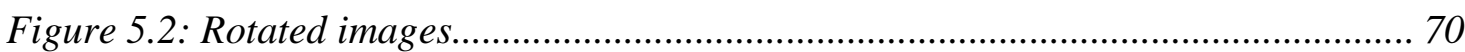




\section{LIST OF TABLES}

Table 2-1: Approximate values of $T_{1}$ and $T_{2}$ for different tissues at 1.0 Tesla............... 10

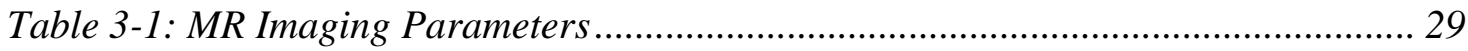

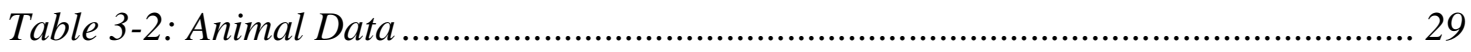

Table 4-1: Gabor filter and threshold parameters for histological images ..................... 56

Table 4-2: Gabor filter and threshold parameters for rabbit brain MRI ....................... 59

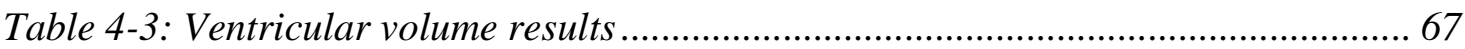




\section{CHAPTER 1: INTRODUCTION}

Image Segmentation is the process of extracting the desired attributes from an image. The segmentation of anatomical structures from a medical image is critical because of its important applications in the medical arena. Some more common applications include brain segmentation into grey matter (GM), white matter (WM) and cerebrospinal fluid (CSF) [7],[8], locating the ventricles (i.e. fluid filled spaces) in the brain and tracking the left ventricle location in the heart from an echocardiogram sequence [3]. Segmentation of brain structures can be crucial for the study of neurological disorders as volume measurements of brain structures may be correlated with disease severity.

The processing of $2 \mathrm{D}$ or $3 \mathrm{D}$ images allows non-invasive isolation of these regions/structures of interest. In this thesis, I have focused on the problem of automated extraction of ventricles from whole head rabbit MRI, with a particular emphasis on the Lateral Ventricles and the Third Ventricle. Changes in ventricular volume are observed in Alzheimer's disease (AD) [9]. In order to understand the importance of Magnetic Resonance Imaging (MRI) as a research tool in the diagnosis of $\mathrm{AD}$, it is helpful to understand a little bit about the nature of the illness and pathology of AD.

\subsection{ALZHEIMER'S DISEASE}

Alzheimer's disease (AD) is a brain disorder named after Dr. Alois Alzheimer [10]. It progressively results in gradual memory loss, and the inability to perform daily activities. It is the most common form of dementia among older persons involving the parts of brain that are involved in thought, memory and language. At the same time, AD is not a part of normal aging. Amyloidal plaques and neurofibrillary tangles [11] are observed post mortem tissue samples from the AD subjects. These plaques and tangles are the hallmarks of AD today. Thus a visible change in brain structure is often observed in AD patients. The brain cells, or neurons, are affected by amyloid in areas of the brain that are vital to memory and other mental abilities. The structural changes are related to memory and functional loss. 
The ventricles are CSF filled structures in the center of the brain. They are bordered by gray and white matter structures that are often affected by diseases associated with: deep white matter, the corpus callosum, hippocampus, amygdala, caudate nucleus, and thalamus. All the above structures are subjected to atrophy (a decrease in size of the brain caused by disease) in the presence of dementia. In Alzheimer's disease, striking changes occur in the brain regions, particularly in variation of the size of the ventricles which can occur in a number of brain diseases, such as Alzheimer's disease [4],[5], and schizophrenia [16], [17] for example.

Memory impairment is the earliest and most severe manifestation in AD. Early diagnosis of dementia is critical for treatment to be started before symptoms and clinical signs of AD are firmly entrenched [18]. Brain imaging can assist in making a differential diagnosis if the abnormality is caused by aging or dementia. Previous research indicates that Magnetic Resonance Imaging (MRI) may be more helpful in the diagnosis of differential forms of degenerative dementia [19] and more sensitive to early and preclinical cases of $\mathrm{AD}$; however, this is still controversial.

\subsection{MOTIVATION AND THE PROBLEM}

The process of segmentation of cerebral ventricles from MRI images is complex and difficult. The lateral and/or third ventricles in the rabbit brain are very small in size compared to the Human brain as shown in Figure 1.1. Hence, manual segmentation is time consuming, tedious, and requires expert supervision. The successful detection of subtle differences in ventricular volume requires an accurate segmentation algorithm. Because of the ventricle structure is small; ventricle extraction becomes difficult in low contrast images. Further, there is no available rabbit atlas that can be used as prior information for segmentation. Since there is no prior information available, standard intensity based and/or probabilistic segmentation methods like Standard Parametric Mapping (SPM) also failed. Currently, no texture based segmentation exists for ventricle extraction in Rabbit Brain. 


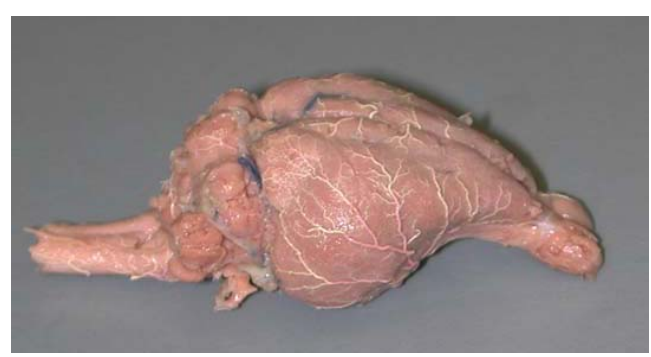

a) Rabbit Brain

approx Rabbit brain length $=5 \mathrm{~cm}$ and approx brain weight $=12 \mathrm{~g}$ for approx body length $=30 \mathrm{~cm}$ and approx body weight $=2500 \mathrm{~g}$

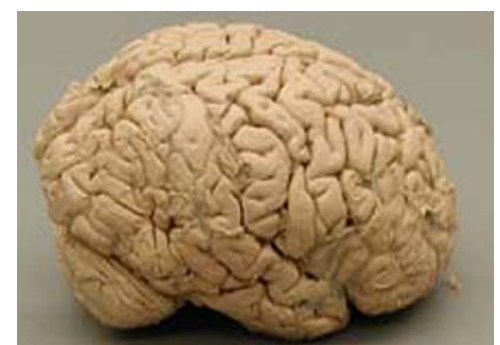

b) Human Brain

approx Human brain length $=15 \mathrm{~cm}$ and approx brain weight $=1400 \mathrm{~g}$ for

approx body length $=170 \mathrm{~cm}$ and approx body weight $=70,000 \mathrm{~g}$

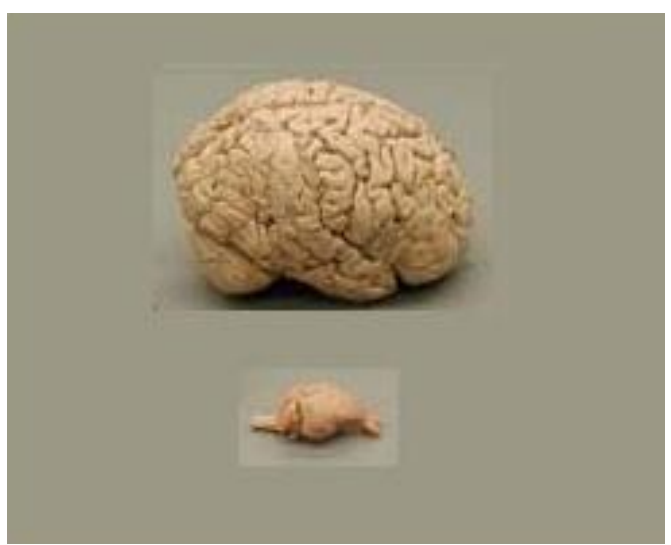

c) Size comparison

Figure 1.1: Comparison of rabbit brain against human brain [64] 
In this thesis, we present a method to measure ventricle volume based on texture based segmentation from MRI data for an animal model of AD. Hence, the primary goal is to present a fully automatic algorithm for the segmentation of rabbit brain ventricles from whole head rabbit MRI data.

\subsection{THESIS OBJECTIVE}

The main objectives of this thesis are summarized as follows:

a) To test the feasibility of using Gabor wavelets to extract texture based features in rabbit brain MRI.

b) To determine the appropriate parameters for the rabbit brain MRI images by developing the method on a histological high contrast set of rabbit brain images.

c) To develop an automated method for extracting ventricular structures from wholehead rabbit MRI, so as to extract ventricles only from whole head rabbit MRI.

d) To automate the seed point selection process from the brain ROI with ventricles.

e) To automatically extract ventricle using Gabor wavelets under supervision.

f) To determine the volume of the ventricles based on the segmentation results.

\subsection{THESIS CONTRIBUTION}

In this thesis, we have:

a) Studied the performance of Gabor wavelets on Brain tissues, White Matter (GM) and Gray Matter (GM) in different imaging modalities.

b) Implemented an automated ventricle extraction algorithm from histological rabbit brain images using Gabor wavelets.

c) Extracted ventricle from Rabbit Brain MRI through a semi-automated method.

d) Implemented automated ventricle extraction process. 


\subsection{THESIS ORGANIZATION}

This thesis is organized in five chapters. Chapter 1 describes the motivation and objectives of this research. Contributions of this research have also been summarized. Chapter 2 provides a brief introduction and overview of MRI, Gabor wavelets, and its related work in Medical Imaging. Chapter 3 discusses the different calculations used in each step for this work. Chapter 4 presents results of the different stages described in the previous chapter. Chapter 5 concludes the thesis, examining how each of the objectives have been met, compares and contrast this method to previous segmentation methods and provides some ideas for future work. 


\section{CHAPTER 2: BACKGROUND AND LITERATURE REVIEW}

\subsection{RABBIT BRAIN MODEL}

Brain anatomy has great importance in neuroscience research. Direct research on human brain is often limited by several factors. Consequently, animal models have traditionally offer an alternative, wherein the physiopathologic aspects of the disease can be simulated, invasive measurements can be made, variables can be controlled, and statistical data accrued in a short period of time. No animal model can satisfy all of these needs so an appropriate species is chosen that best fits the study.

Rabbits have traditionally been among the most widely used animals in experimental studies in basic and clinical medical sciences because of their size, similar physiological attributes to humans, and similar disease characteristics.

Rabbits have been used as models for several brain studies such as evaluation of cell viability in MR imaged focused ultrasound (FUS) lesions and cell death zones [20]. The animal model of Alzheimer's disease used in this thesis is described in the Schreurs and Sparks PNAS article [21].

\subsection{MAGNETIC RESONANCE IMAGING (MRI)}

Magnetic resonance imaging (MRI) is an imaging technique used to visualize the human anatomy. MRI works using the principles of nuclear magnetic resonance (NMR), a spectroscopic method used to obtain the properties of molecules. MRI is NMR which is spatially based - designed to produce images of the tissue.

It has been over 60 years, since Felix Bloch and Edward Purcell discovered the Nuclear Magnetic Resonance (NMR) phenomenon in 1946. NMR was developed and used for physical and chemical molecules until 1970. Raymond Damadian found using MRI that nuclear magnetic relaxation times of tissues and tumors differed in 1971. Thus 
the scientists were motivated to develop imaging that uses NMR for detecting disease. In 1973, Paul Lauterbur first demonstrated MRI (NMR which was displayed in spatial arrays i.e. images) on small test tubes filled with water. In 1974, Raymond Damadian started a company, the Meville, N.Y.-based Fonar Corp and made MRI imaging device. In 1975 Richard Ernst proposed magnetic resonance imaging using phase and frequency encoding, and the Fourier Transform which is the basis of modern MRI techniques. After few years, in 1977, Raymond Damadian demonstrated MRI of the whole body. Peter Mansfield developed the echo-planar imaging (EPI) technique in the same year. Paul Lauterbur and Sir Peter Mansfield were jointly awarded the Nobel Prize in Medicine in 2003 for their discoveries concerning magnetic resonance imaging. Thus MRI is a growing science.

The past 15 years have witnessed an explosion of information regarding the role of magnetic resonance imaging in assessing pathologic conditions. MRI is considered much safer then x-ray or computed tomography (CT) as it uses only non-ionizing electromagnetic fields and has rapidly evolved into an accepted modality for medical imaging of disease processes throughout the body. MRI provides a three dimensional digital representation of tissue characteristics that can be obtained in any spatial orientation. Since its inception, MRI has expanded beyond a tomographic imaging technique; it is also capable of true volume imaging.

MRI has become a preferred method for diagnosing problems in many parts of the body. MRI is very helpful, providing noninvasive imaging of soft tissue, such as organs, muscle, cartilage, ligaments, and tendons. While x-rays or CT are best for viewing bones, MRI can identify and show the difference between healthy and unhealthy tissue. With MRI, it is often possible to diagnose disease at very early stage, and before it is visible by any other means. Because most disease are best treated when found early, MRI scans are becoming increasingly useful [18], [19]. 


\subsubsection{Principles of MRI}

The human body is primarily composed of water and fat. Thus it is approximately $63 \%$ hydrogen atoms. A hydrogen nucleus is a single proton. The proton possesses a property called spin which creates a small magnetic field and causes the nucleus to produce an NMR signal. Hence MRI of the human body primarily images the NMR signal from these hydrogen nuclei.

The basic principles of forming an MRI dataset [1] can be stated as follows:

a) the protons in the body are placed in a strong magnetic field;

b) the protons are excited by a radio frequency $(\mathrm{RF})$ energy pulse;

c) after a delay those same protons emit the RF energy;

d) the resulting emitted RF energy from that tissue is sampled;

e) the RF energy is collected while additional gradient magnetic fields are applied to localize the signal;

f) the sampled data are reconstructed to form a two (or three) dimensional image of the particular region of interest in the body being studied

Proton density describes the number of protons in one unit volume. Protons can be considered as tiny bar magnets with north and south poles. Protons in the absence of magnetic field are randomly oriented. When the proton is placed in an external magnetic field $B_{0}$, it aligns itself with the external field and reaches an equilibrium magnetization $M_{0}$. This can be described by the following Bloch equation.

$$
\frac{d M}{d t}=\mu M \times B
$$

The magnetization is proportional to the external magnetic field. The magnetic moments exhibit the property of precessing in the field. The frequency of precession is given by the Larmor frequency:

$$
f_{L}=\frac{\gamma B_{0}}{2 \pi}
$$


For the proton, $\frac{\gamma}{2 \pi}=42.58 \mathrm{MHz} /$ Tesla, thus the Larmor frequency will be in radiofrequency region $(40-50 \mathrm{MHz})$. The Larmor frequency is proportional to the magnetic field strength $B_{0}$.

The proton excited to a higher energy state, then emits RF energy as photons to return to the lower energy state. Thus the particle undergoes a transition between the two energy states. After the RF is emitted, the magnetization returns back to the equilibrium position. The protons emit MRI signals during relaxation and the signals are collected in the presence of localized gradient fields to generate a two dimensional MRI image [2].

The relaxation time can be divided into two components:

- $T_{1}$, Spin-lattice relaxation time.

- $T_{2}$, Spin-spin relaxation time.

Each of these components has a corresponding effective relaxation time:

- $T_{1}^{*}$

- $T_{2}^{*}$

\subsubsection{Relaxation Constants}

At equilibrium, the net magnetization vector lies along the direction of the applied magnetic field $B_{0}$ and is called the equilibrium magnetization $M_{0}$. In this configuration, the $\mathrm{Z}$ component of magnetization $M_{z}$ equals $M_{0}$. The return of $M_{z}$ to equilibrium is described by the time constant, the spin lattice relaxation time $\left(T_{1}\right)$. The $T_{1}$ is an exponential process and is typically in seconds. $T_{1}$ results from the interaction between the protons and the surrounding environment within 2-6 microns. It is short for fat and long for water as shown in Table 2.1. 
$T_{2}$ characterizes the decay of transverse magnetization and represents the dephasing of the spins. $T_{2}$ is called the spin-spin relaxation time. $T_{2}$ is caused by the result of interactions between neighboring protons and are diffusion-based. $T_{2}$ is always less than or equal to $T_{1}, T_{2}$ behaves in the same manner as $T_{1}$ for fat and water. Hence, the intensity at each point of an MR image is a combination of the proton density $\rho, T_{1}$ and $T_{2}$.

The tissue inhomogeneities, air bubbles, or tissue interfaces reduce the relaxation constant and $T_{2}$ is called the effective $T_{2}^{*} \cdot T_{2}^{*}$ includes dephasing due to inhomogeneities and hence is much shorter than $T_{2}$.

$$
\frac{1}{T_{2}^{*}}=\frac{1}{T_{2}}+\frac{1}{T_{2 i n \text { hom } o}}
$$

The Bloch equation including the relaxation constants $T_{1}$ and $T_{2}$ is given by:

$$
\frac{d M}{d t}=M \times \gamma B-\frac{\left(M_{x} i+M_{y} j\right)}{T_{2}}-\frac{\left(M_{z}-M_{0}\right) k}{T_{1}}
$$

where

$M_{x}, M_{y}, M_{z}$ is magnetization in the $x, y, z$ plane respectively

$i, j, k$ are unit vectors in $x, y, z$ directions respectively

Table 2-1: Approximate values of $T_{1}$ and $T_{2}$ for different tissues at 1.0 Tesla

\begin{tabular}{|c|c|c|}
\hline Tissue & $\mathbf{T}_{\mathbf{1}}$ (msec) & $\mathbf{T}_{\mathbf{2}}$ (msec) \\
\hline White Matter & 390 & 90 \\
\hline Gray Matter & 520 & 100 \\
\hline Cerebrospinal Fluid (CSF) & 2000 & 300 \\
\hline Muscle & 600 & 90 \\
\hline Fat & 180 & 50 \\
\hline Liver & 270 & 180 \\
\hline Blood & 800 & \\
\hline
\end{tabular}


A few important parameters associated with the MRI system include:

Field of View (FOV): It is defined as the size of a two dimensional spatially sampled area which makes the image. It is the square image area that contains the object of interest to be measured. The smaller the FOV, the higher the resolution and the smaller the voxel size but the lower the measured signal. The right choice of FOV is important for MR image quality and depends on the body part being imaged [12]. It ranges from 10 to $50 \mathrm{~cm}$ for most machines.

Slice Thickness: It is the thickness of an imaging slice. For optimal image quality it is important to choose the best fitting slice thickness for an examination. When a small item of interest is contained within the slice thickness with other tissue of differing signal intensity then the resulting signal displayed on the image is a combination of these two intensities. If the slice is the same thickness or thinner than the small structure of interest, only that structures signal intensity is displayed on the image [12]. It ranges mainly from 3 to $15 \mathrm{~mm}$.

Matrix Size: All digital techniques consider images to be divided into a matrix of pixels - or two dimensional individual image components. For each pixel, the MR signal intensity is represented on an 8-bit gray scale. The pixels together form a two dimensional image matrix. By contrast, voxels are three dimensional entities that are sampled in the two dimensionality image space. Three dimensional voxels are represented by two dimensional voxels making up the MR image with the third dimension being filled out by multiple slices. The most commonly used matrix size is $256 \times 256$

Number of Excitations (NEX): Every individual signal needed to generate an MRI image can be acquired once or several times with repeated excitations generating an average image. With more excitations the average error in the measurements decreases resulting in more precise measurements. However, this lengthens image acquisition time 
which can then result in imaging artifacts when the subject moves. The number of excitations typically ranges from 1 to 6 .

Flip Angle: The flip angle $\alpha$ is used to define the angle of excitation for a field echo pulse sequence. It is the angle to which the net magnetization is rotated or tipped relative to the main magnetic field direction via the application of a RF excitation pulse at the Larmor frequency. It is also referred to as the tip angle, nutation angle or angle of nutation. Flip angles between $0^{\circ}$ and $90^{\circ}$ are typically used in gradient echo sequences, $90^{\circ}$ and a series of $180^{\circ}$ pulses in spin echo sequences and an initial $180^{\circ}$ pulse followed by a $90^{\circ}$ and a $180^{\circ}$ pulse in inversion recovery sequences.

\subsubsection{Pulse Sequences}

In the sections following, the three pulse sequences are used to collect MRI images for this thesis as described, a fast-spin echo, spoiled gradient echo, and time of flight angiography. Related background material needed to explain the pulse sequences is presented first.

A pulse sequence is a series of RF pulses and/or magnetic field gradients applied to a spin system (a sample material like biological tissue) to produce a signal representative of some property of the spin system which is then Fourier transformed to form the MR image. The essential component of pulse sequence is the RF pulse. The various gradients, slice selection gradient, phase encoding gradient and frequency encoding gradient are used in MR imaging. They are used with selective excitation to select a region for imaging and also to encode the location of MR signals received from the object being imaged. 


\subsubsection{Spin echo imaging}

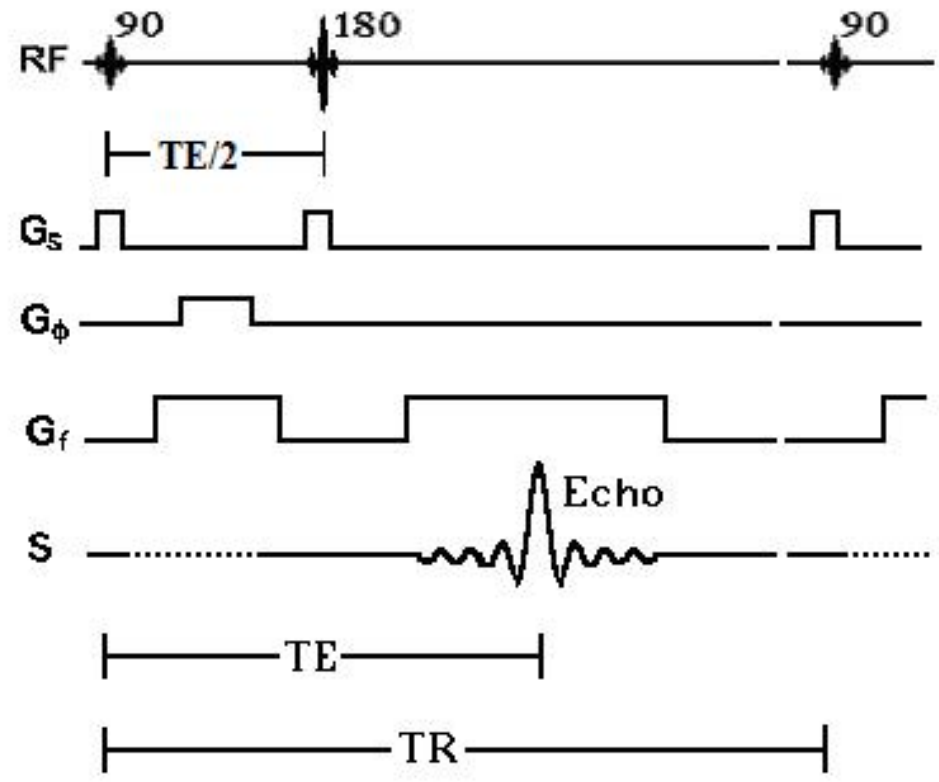

Figure 2.1: Spin echo pulse sequence

This is the most commonly used pulse sequence. The spin-echo pulse sequence shown in Figure 2.1 commences with a 90 degree RF pulse which shifts the magnetization vector into the transverse plane yielding measurable free induction decay (FID) signal. A slice selection gradient is applied in conjunction with the RF pulse to select the section of material to be sampled. After a period of time equal to TE/2, an $180^{\circ}$ pulse is applied in conjunction with the slice selection gradient. A phase encoding gradient is applied between the $90^{\circ}$ and $180^{\circ}$ pulses.

The frequency encoding gradient is applied at a time TE/ 2 after the $180^{\circ}$ pulse during the time that echo is collected. The recorded signal is the echo. The FID, which is found after every $90^{\circ}$ pulse, is not sampled. A dephasing gradient is applied along the same direction as the frequency encoding gradient. It dephases the spins so that they will rephrase by the center of the echo. This gradient in effect prepares the signal to be at the edge of k-space by the start of the acquisition of the echo. 
The two variables of interest in spin echo sequences are the repetition time (TR) and the echo time (TE). The repetition time (TR) is the time interval between the beginnings of two consecutive data acquisition. The echo time (TE) is the time between the beginning of the sequence and the middle of the echo.

The entire sequence is repeated every TR until all the phase encoding steps has been recorded. It can be seen that the slice selection gradient is turned on again each TR during the 180 degree RF pulse.

\subsubsection{Fast spin echo}

The fast spin-echo (FSE) pulse sequence is the subset of spin echo pulse sequence. This sequence comprises a series of rapidly applied $180^{\circ}$ rephrasing pulses and multiple echoes (echo-trains), changing the phase encoding gradient for each echo. The echo time (TE) varies from echo to echo in the echo train. The echoes in the center of the $\mathrm{K}$-space mainly produce the type of image contrast and the periphery of K-space determines the spatial resolution.

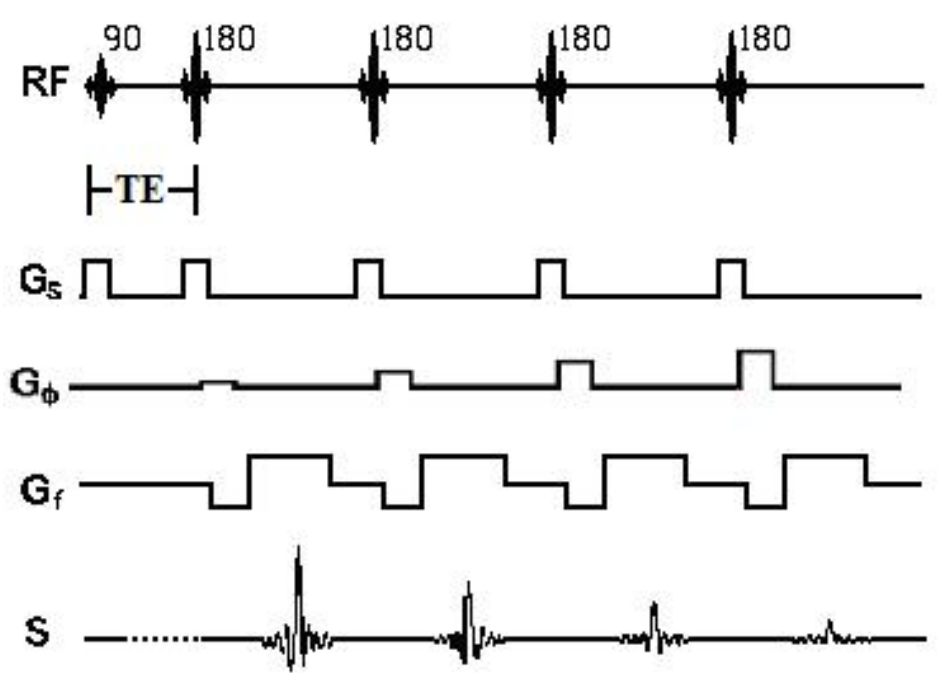

Figure 2.2: Fast spin echo imaging 
The FSE pulse sequence along with the gradients is shown in Figure 2.2. The most important difference between the FSE sequence and a conventional spin-echo sequence is that groups of consecutive echoes, within an echo train, are acquired. Fast spin echo allows reduced acquisition times.

\subsubsection{Gradient recalled echo sequence}

Gradient recalled echo (GRE) sequence reverses the polarity of the gradient field rather than using a 180 degree RF pulse to invert the spins to generate an echo signal. A slice selective RF pulse is applied to the imaged object. This RF pulse typically produces a rotation angle of between $10^{\circ}$ and $90^{\circ}$. A slice selection gradient is applied with the RF pulse. Contrast can be manipulated by changing the RF pulse angle. A phase-encoding gradient is applied next as shown in Figure 2.3.

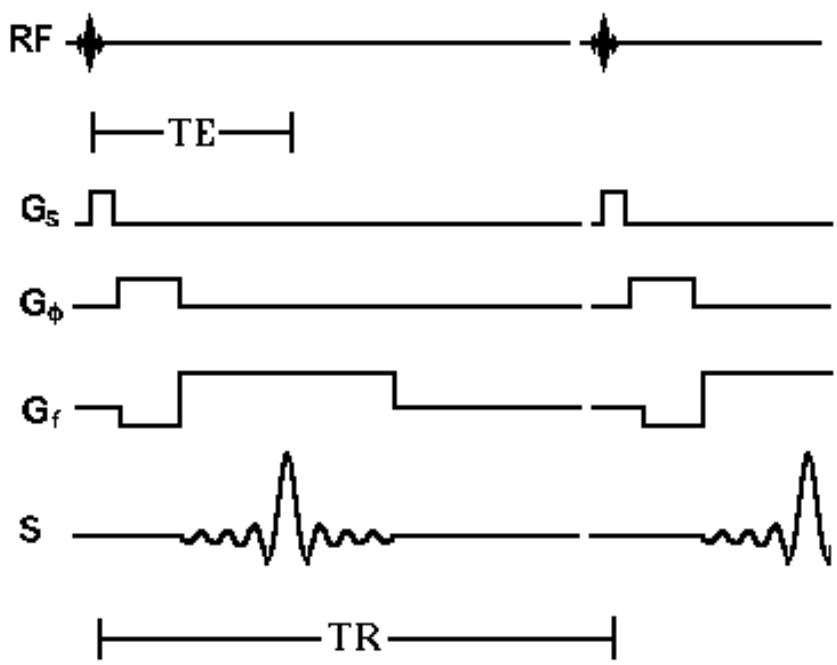

Figure 2.3: Gradient recalled echo pulse sequence

A dephasing frequency encoding gradient is applied at the same time as the phase encoding gradient so as to cause the spins to be in phase at the center of the acquisition period. This gradient is negative in sign from that of the frequency encoding gradient turned on during the acquisition of the signal. An echo is produced when the frequency 
encoding gradient is turned on because this gradient refocuses the dephasing which occurred from the dephasing gradient. The sequence is repeated every TR seconds. The TR period could be as short as tens of milliseconds.

Imaging with a gradient echo is intrinsically more sensitive to magnetic field inhomogeneities. The use of a small flip angle and of a gradient for the refocusing of magnetization vectors gives this sequence a time advantage. Therefore it is widely used for fast scan images, including 3D acquisitions.

\subsubsection{3D-Spoiled Gradient Echo Sequence}

3D-spoiled Gradient Echo Sequence (SPGR) is the subset of GRE. SPGR use a spoiler gradient on the slice selection gradient during the end module to destroy any remaining transverse magnetization after the frequency encoding gradient, which is the case for short repetition times. Hence, only z-magnetization remains during a subsequent excitation.

\subsubsection{Time of Flight Angiography}

Time of Flight (TOF) imaging is based on conventional gradient echo scanning with flow compression. MRI angiogram images are created by repeatedly exciting a predefined volume of anatomy until the stationary tissue is partially saturated and the signal from the tissue is suppressed. Blood flowing into the predefined volume of anatomy is not saturated but fully magnetized by the main magnetic field and yields a stronger signal. In the resulting image, the blood appears bright and the stationary tissue is suppressed. This phenomenon is called a flow-related enhancement.

The time of flight angiography is used for the imaging of vessels. Usually, the sequence type is a gradient echo sequences with short TR as observed from Figure 2.4, acquired with slices perpendicular to the direction of blood flow. The magnetic field 
gradients $G_{s}, G_{y}$ and $G_{z}$ are the frequency-encoding, phase-encoding and slice-selection gradients, respectively.

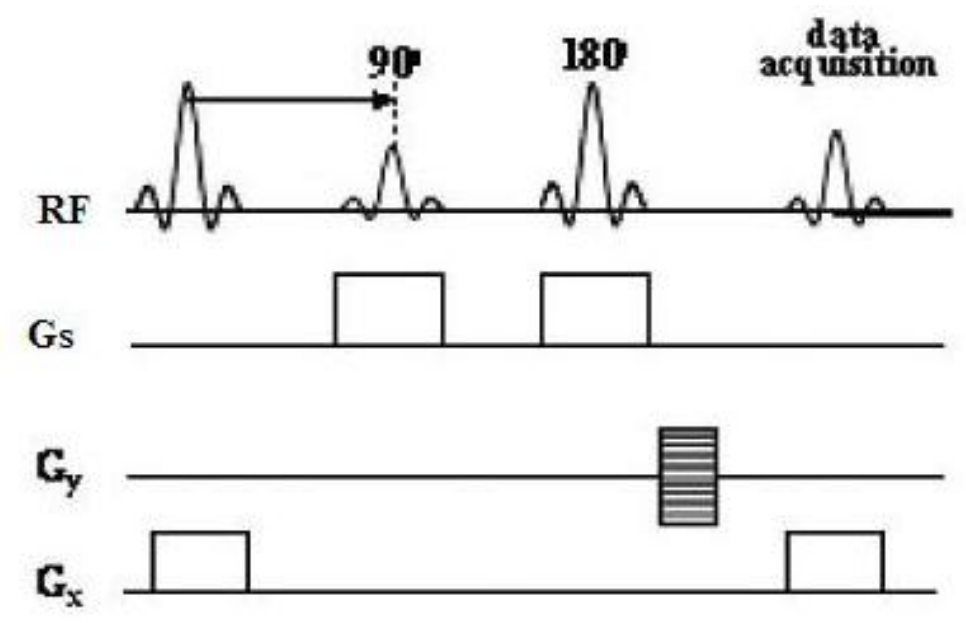

Figure 2.4: TOF pulse sequence

The source of diverse flow effects is the difference between the unsaturated and presaturated spins and creates a bright vascular image without the invasive use of contrast media. Flowing blood moves unsaturated spins from outside the slice into the imaging plane. These completely relaxed spins have full equilibrium magnetization and produce (when entering the imaging plane) a much higher signal than stationary spins if a gradient echo sequence is generated. This flow related enhancement is also referred to as entry slice phenomenon, or inflow enhancement.

Performing a presaturation slab on one side parallel to the slice can selectively destroy the MR signal from the in-flowing blood from this side of the slice. This allows the technique to be flow direction sensitive and to separate arteriograms or venograms. When the local magnetization of moving blood is selectively altered in a region, e.g. by selective excitation, it carries the altered magnetization with it when it moves, thus tagging the selected region for times on the order of the relaxation times. 
For maximum flow signal, a complete new part of blood has to enter the slice every repetition (TR) period, which makes time of flight angiography sensitive to flowvelocity. The choice of TR and slice thickness influences the performance of the TOF sequence. The use of sequential 2D Fourier Transform (2DFT) slices, 3DFT slabs, or multiple 3D slabs (chunks) are depending on the coverage required and the range of flowvelocities. 3D TOF MRA is routinely used for evaluating the Circle of Willis.

\subsection{INTRODUCTION TO TEXTURE-BASED SEGMENTATION}

Texture can be defined as a measure of the variation of the intensity of a surface, quantifying properties such as smoothness, coarseness and regularity [66]. It is often used as a region descriptor in image analysis and computer vision. There are three principal approaches used to describe texture: statistical, structural and spectral. Statistical techniques characterize texture by the statistical properties of the grey levels of the points comprising a surface. Typically, these properties are computed from the grey level (histogram) or grey level (cooccurrence matrix) of the surface. Structural techniques characterize texture as being composed of simple primitives called "texels" (texture elements), that are regularly arranged on a surface according to some rules. Spectral techniques are based on properties of the Fourier spectrum and describe global periodicity of the grey levels of a surface by identifying high energy peaks in the spectrum.

Texture segmentation is required in many image analysis and computer-vision applications. It plays a vital role in the interpretation and understanding of real world images. Texture segmentation can be defined as partitioning an image into differently textured regions. Textures are also modeled as irradiance patterns containing localized spatial frequencies, orientation, and phase characteristics [61]. Figure 2.5 shows different textures from Broadatz album. 


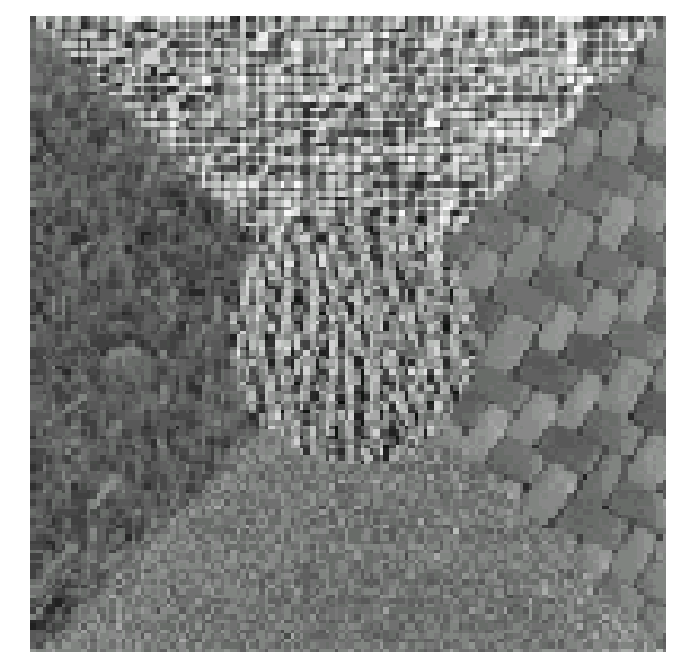

\section{Figure 2.5: Different textures from Broadatz album [65]}

There are various methods for analyzing image textures. In this work, we focus on filter-based approaches. Each texture can be located by filtering the image with multiple band-pass filters each tuned to the dominant frequency and orientation of the texture. The filters are designed to focus on a particular range of spatial frequencies and on local spatial interactions. Hence, they constitute a joint space/spatial-frequency decomposition. The frequency is viewed as a local phenomenon that varies with position in an image. The effectiveness of the multiresolution filtering approach to texture segmentation is well recognized [34], [44], [48], [50] and [61]. Though several filters could perform the joint space/spatial-frequency decomposition, the Gabor filtering method is particularly [40] [42], [44] - [48].

The motivation for using Gabor filters in extracting texture image features is that they have been shown to be optimal in the sense of minimizing the joint two-dimensional uncertainty in space and frequency [31]. Gabor filters are a collection of wavelets, where each wavelet can capture the energy of one particular spatial frequency and in one specific orientation [35]. Hence, Gabor filters are also considered to be orientation and scale tunable edge and line detectors.

The micro-feature statistics extracted from the Gabor transform can be used to characterize the underlying texture information. Gabor wavelets are widely used in the 
area of image analysis applications, which includes texture classification [58],[60],[62] and texture segmentation [34], [44], image recognition and retrieval [31], image registration, and motion tracking [32].

\subsubsection{Gabor Wavelets}

For a given two-dimensional image $I(x, y)$ with size $N \times N$, the discrete Gabor wavelet transform is given by [31]

$$
E_{m n}(x, y)=\sum_{k} \sum_{l} I(x, y) g_{m n}^{*}(x-k, y-l)
$$

where $k, l$ are filter mask variables and $m, n$ are scale and orientation parameters, respectively. The function $g_{m n}^{*}$ is the complex conjugate of a two dimensional Gabor function. The Gabor functions are obtained for a specific $m$ and $n$ pair. This gives them "tenability" to textures.

In general, the two dimensional Gabor function is given by

$$
g(x, y)=\frac{1}{2 \pi \sigma_{x} \sigma_{y}} \exp \left[-\frac{1}{2}\left(\frac{x^{2}}{\sigma_{x}^{2}}+\frac{y^{2}}{\sigma_{y}^{2}}\right)+j 2 \pi F x\right]
$$

where $\sigma_{x}, \sigma_{y}$ characterize the spatial extent and bandwidth and $F$ is the modulation frequency. A class of self-similar functions known as Gabor wavelets is obtained by appropriate dilation and rotation of $g(x, y)$ using the generating function

$$
g_{m n}(x, y)=a^{-m} g\left(x^{\prime}, y^{\prime}\right), \quad a>1
$$

where $m=0,1,2, \ldots, \eta_{s}-1, n=0,1,2, \ldots, \eta_{d}-1, \eta_{s}=$ the number of scales, and $\eta_{d}=$ the number of orientations in the multiresolution decomposition. The Gabor wavelets for 4 scales and 8 orientations are shown in Figure 2.6. The new position variables are given by

$$
\begin{aligned}
& x^{\prime}=a^{-m}(-x \cos \theta+y \sin \theta) \\
& y^{\prime}=a^{-m}(-x \sin \theta+y \cos \theta) \\
& \text { and } \quad \theta=\pi n / \eta_{d}
\end{aligned}
$$



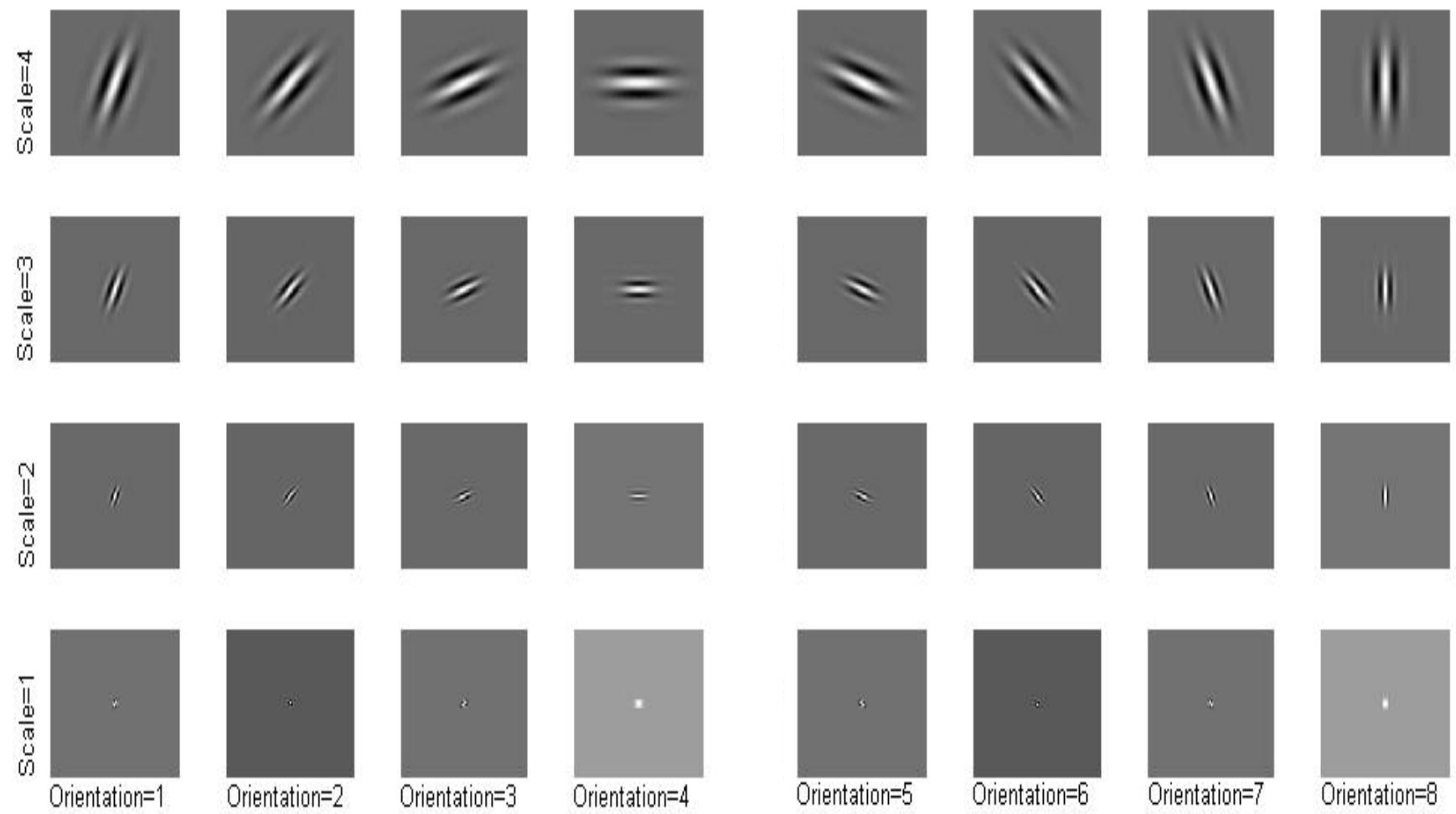

Figure 2.6: Gabor wavelets for 4 scales and 6 orientations 
The variables in the above equations are defined by:

$$
\begin{aligned}
& a=\left(\frac{U_{h}}{U_{l}}\right)^{\frac{1}{\eta_{s}-1}} \\
& F_{m n}=U_{h} \\
& \sigma_{x_{m n}}=\frac{(a-1) U_{h}}{(a+1) \sqrt{2 \ln 2}} \\
& \sigma_{y_{m n}}=\tan \left(\frac{\pi}{2 \eta_{d}}\right) \times \frac{\left[U_{h}-2 \ln \left(\frac{\sigma_{x_{m n}}}{U_{h}}\right)\right]}{\sqrt{\left[2 \ln 2-\frac{(2 \ln 2)^{2} \sigma_{x_{m n}}^{2}}{U_{h}}\right]}}
\end{aligned}
$$

The parameters $U_{h}$ and $U_{l}$ are, respectively, the upper and lower center frequencies.

\subsection{GABOR WAVELETS IN MEDICAL IMAGING}

Texture is one of the important image features that provide information for identifying surfaces and objects in image [22]. It is a major component of image processing, and is fundamental to many applications such as remote sensing, quality inspection, medical imaging. Extraction of proper features having good discrimination properties is the first crucial step. Several methods have been developed over time for feature extraction. Such methods include: Gray level co-occurrence matrix (GLCM) [23], Gabor filters [7], [23], [25], and wavelet transform [25].

Gabor Wavelets have been used for the classification of diffused liver diseases [27]. The traditional method used to identify normal from abnormal liver tissue involves pattern recognition of certain characteristics from the image by comparing them to images with known different pathologies. Some diseases can yield similar images leading to an inability to make a definitive diagnosis. Hence, researchers have attempted to develop non-invasive quantitative measures which help solve this problem. One of these measures is the identification of the three kinds of liver abnormalities: cyst, hepatoma and cavernous based on Gabor filters [28]. The current method to diagnose liver disease is an 
invasive method depending on the needle biopsy of the liver. The sampling error in the biopsy specimen can bias the pathological measurement of these diseases. Hence a reliable noninvasive method of evaluating histological changes in sonograms will be a major advance in diagnosis of chronic liver diseases [27].

Gabor filters have the ability to model the frequency and orientation sensitivity characteristic of the human visual system. Xie et. al [33] has adopted features taken from Gabor filters and shape to perform a study of segmentation of kidney from ultrasound images. Ferrari et. al analyzed the asymmetry in tissue sampled in mammograms using the directional property of the Gabor wavelets [29]. Another study involved detecting edges in the ultrasonic images using Gabor wavelets [29].

\subsection{GABOR WAVELETS IN THE ANALYSIS OF RABBIT BRAIN IMAGES}

There is a large demand for useful image analysis tools to aid the investigations in the field of neuroscience [36]. There are several studies that have concentrated in this issue in the area of biomedical image processing often in MRI or Positron Emission Tomography (PET) images. Such studies require high operational and computational costs. Various staining techniques have advanced making the anatomical studies of brains easier. Hence visualization of subcortical areas within the sliced brain tissues is not difficult. Here the earlier work on the investigation on applying the Gabor wavelet-based image encoding and processing technique to the Nissl-stained rat brain slice images is discussed in brief [37].

Gabor wavelet-based approach has been successfully applied to the task of face recognition from two dimensional grayscale black and white images of faces in the past [38]. Researchers aimed at applying the method to other classes without object-specific optimizations. However, the face recognition method had to be modified to test with the rat brain images for slice classification and automatic slice registration. This study aimed at investigating the feasibility of analyzing the $2 \mathrm{D}$ brain images created by the Nissl- 
stain. The Nissl-stained rat images differ greatly from human face images as shown in Figure below.

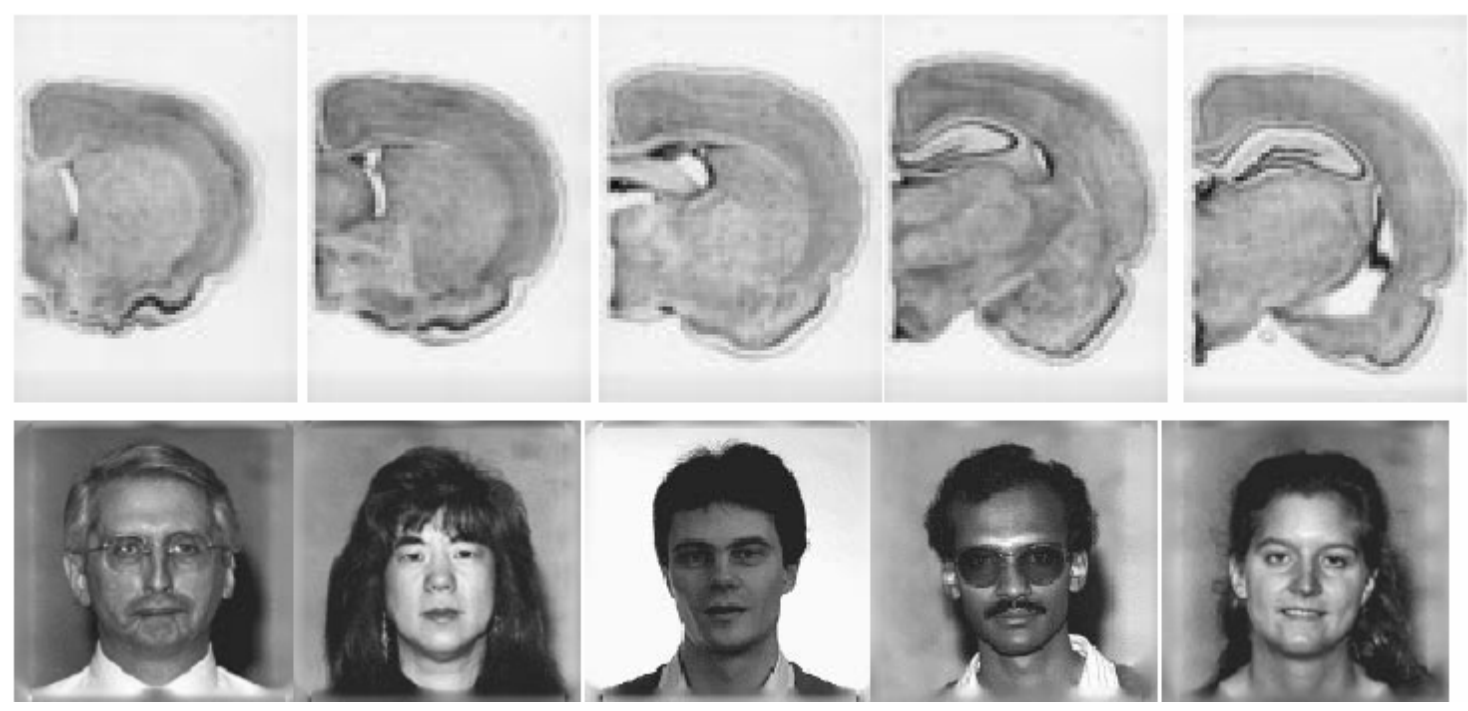

Figure 2.7: Comparison of the nissl-stained images and facial images [37]

The brain images used in the study are electronically scanned from the original positive copies of the Swanson's Rat Brain Atlas [39]. 21 images from plate 15 to 35 are used in this study. A filtering operation with Gabor kernels helps extracting the relevant information of objects in the images. The resulting Gabor jet-based object representation allows a pixel to be associated with a feature vector called jet containing the spatial frequency information of surrounding local region of pixel.

The classification test is performed, for all the 21 entries. One entry from the album is matched against other 21 entries. The album entry most similar to an input is called the best match. A correct classification is done when the best match is one of the two adjacent slices of the probe. The two algorithms used for searching the best match are averaging and voting.

The modified classification system with the voting algorithm performed very well with $100 \%$ success rate was shown empirically. The preliminary system for the automatic 
registration performed satisfactorily. Similarity analyses results indicated that the successful performance appeared to be attributes to information in the high-frequency range (subcortical level) rather than in the low-frequency range (cortical contour). Thus the results motivate the Gabor wavelet-based method for further image processing systems. Our work is concentrated on using Gabor wavelet-based method for a different task, ventricular measurements. This study was performed on rabbit brain MRI rather than the histological images.

\subsection{VENTRICULAR MEASUREMENTS AND ANALYSIS}

The earlier works on ventricular measurements and analysis on human brain MRI are discussed in this section. Ventricular volume is a frequently examined feature of human brain development, aging as well as pathology. Changes in CSF ventricular volume also have been shown to have clinical significance in patients with Alzheimer Disease [51]-[53]. There are several techniques for ventricle volume quantification that rely on different combination of image segmentation techniques.

On T1-weighted MR images, dark regions corresponding to cerebrospinal fluid (CSF) could be selected and segmented by thresholding the image. On T2-weighted MR images, a threshold could be used to pick high signal from CSF. The threshold can be selected manually by supervision or by intensity histogram analysis. Using the histogram analysis, a binary image mask can be created where the ventricles appear bright and the entire non-ventricular regions appear dark. Then a comparison between the thresholded binary image and gray scale image is required for segmentation verification.

One of the other segmentation techniques involves edge detection and linking discontinuities in the edges to extract the ventricles. This process is tedious and requires manual intervention. Semi-automated procedures extract ventricles using a seed point and region growing methods. Many semi-automated methods are subjective, prone to operator errors, and can be labor intensive. 
Multispectral image segmentation techniques have proved to be robust in extracting regions of the ventricle, but a drawback of these methods is that they involve acquiring the data using more than one MR sequence [55]-[57]. Much of the research in automatic MR brain and brain related segmentation incorporates domain specific knowledge to deal directly with difficult aspects of the problem [58].

MR imaging has made it possible to obtain in vivo 3D images of the human brain non-invasively, thereby allowing large scale of examinations of brains. While for some diagnostic applications a simple counting the number of slices in which the ventricles appear, or even a visual inspection, suffices, objective and specifically quantitative segmentation of the ventricular system is necessary for precise measurement of the ventricular volume [54].

\subsection{OTHER RELATED WORK - CLASSIFICATION AND SEGMENTATION}

The automated segmentation of neuroanatomical structures in multispectral MR microscopy of the mouse brain is discussed here [43]. A total of 21 structures were segmented and included the ventricular system, corpus callosum, hippocampus, caudate putamen, inferior colliculus, internal capsule, globus pallidus, and substantia nigra. Six C57BL/6J male mice approximately 9 weeks in age were used in the study. A multispectral, three-dimensional (3D) MR data, proton density-weighted, T2-weighted and diffusion-weighted images were acquired at $90-\mu \mathrm{m}$ isotropic resolution from a 9.4-T (400 MHz) vertical bore Oxford superconducting magnet. Inter-specimen registration was not required. A 9-parameter affine registration (translation, rotation, and scaling) was applied to each brain for intra-specimen registration.

Each T2-weighted dataset of the six brains was manually traced and completely labeled into 21 neuroanatomical structures using a standard atlas as reference. Spatial information is employed in the form of prior probabilities of occurrence of a structure at a location (location priors). The Bayes theorem is used to compute the maximum posterior probability, the most likely class to which the measured data belong from the probability 
density function of each class and prior probability. The maximum likelihood estimates of mean and covariance matrix at each class at every location in the atlas form the multivariate intensity model for classification.

Pairwise probabilities between structures (contextual priors) are modeled using the Markov Random Field (MRF) concepts. The MRF model favors the arrangement of labels that conform itself by associating them with larger probabilities. Validation using standard morphometry indices shows good consistency between automatically segmented and manually traced data. Results achieved in the mouse brain are comparable with those achieved in human brain studies using similar techniques. The segmentation algorithms show excellent potential for routine morphological phenotyping mouse models. 


\section{CHAPTER 3: METHODS}

The ventricle extraction procedure had to be formulated based on a detailed study of Gabor wavelets. The developmental work was divided into three stages to finally get to the point where the ventricles could be extracted from rabbit brain MRIs. In stage 1, a test was conducted to evaluate the performance of Gabor wavelets on the Rabbit Brain MRI. In stage 2, we continued to study the Gabor wavelets on another type of image set (high contrast greyscale histological images) of rabbit brain. Stage 3 details the work of ventricle extraction from Rabbit Brain MRI. The Gabor wavelet method of feature extraction discussed in Chapter 2 is used in all the three stages. In this chapter the details of the implementation of the method are described.

\subsection{MR DATA ACQUISITION}

The 3D volumetric data generated by MRI have wide ranging applications in medical imaging. The following section gives a brief overview of the MR data acquisition and analysis. The MR pulse sequences used to collect data sets were T2weighted 2DFSE, T1-weighted 3D SPGR and 3D TOF Magnetic Resonance Angiography (MRA) image data sets were used in this study. The MR parameters involved are summarized in Table 3.1 The MRI scans analyzed for this thesis were obtained in six normal rabbits and seven cholesterol-fed rabbits. The rabbits were cared for as described in Schreurs and Sparks [21]. Table 3.2 shows the MR scans performed and number of images acquired for each scans for both the animal groups.

The images were preprocessed as follows before the filtering operation. The images were first converted into 3D datasets (also known as BRIKs) using Analysis of Functional NeuroImages (AFNI) software [62]. The first two dimensions are the in plane $\mathrm{x}$ and $\mathrm{y}$ position, and the third dimension is the slice or $\mathrm{z}$ direction. The BRIKs were made for all the image sets i.e., FSE, FSPGR and TOF. The BRIKs (integer data) were read into a MATLAB (numerical computing environment and programming language 
created by Mathworks) script for performing filtering and extraction. Figure 3.1 below shows the images acquired from three scans.

Table 3-1: MR Imaging Parameters

\begin{tabular}{|c|c|c|c|c|c|c|c|c|}
\hline $\begin{array}{c}\text { Pulse } \\
\text { Sequence }\end{array}$ & $\begin{array}{c}\text { Field- } \\
\text { of- } \\
\text { view } \\
(\mathbf{F O V}) \\
(\mathbf{m m})\end{array}$ & $\begin{array}{c}\text { Slices } \\
\text { thickness } \\
(\mathbf{m m})\end{array}$ & $\begin{array}{c}\text { SR } \\
(\mathbf{m s})\end{array}$ & $\begin{array}{c}\text { TE } \\
(\mathbf{m s})\end{array}$ & $\begin{array}{c}\text { Matrix } \\
\text { Freq*phase }\end{array}$ & $\begin{array}{c}\text { Flip } \\
(\mathbf{d e g})\end{array}$ & NEX \\
\hline 2D FSE T2 & 80 & $20-40$ & 3.2 & 3 & 14.24 & $256^{*} 192$ & 90 & 2,4 \\
\hline 3D FSPGR & 80 & 72 & $1.6 / 0.8$ & 10 & 2.303 & $256^{*} 192$ & 15 & 4.00 \\
\hline 3D TOF & 80 & 110 & $1.6 / 0.8$ & 40 & 6.7 & $512 * 256$ & 25 & 1.00 \\
\hline SPGR & & & & & & & & \\
\hline
\end{tabular}

Table 3-2: Animal Data

\begin{tabular}{|c|c|c|c|c|c|}
\hline \multicolumn{3}{|c|}{ Normal Diet + Distilled $\mathrm{H}_{2}$ ( } & \multicolumn{3}{c|}{ H2 $_{2}$ + 12 ppm Cu } \\
\hline Animal & FSPGR & TOF & Animal & FSPGR & TOF \\
\hline D375 & 72 & 92 & D392 & 72 & 110 \\
D376 & 72 & 110 & D394 & 72 & 110 \\
D377 & 72 & 110 & D397 & 72 & 110 \\
D378 & 72 & 110 & D398 & 72 & 110 \\
D443 & 72 & 80 & D455 & 72 & 76 \\
D444 & 72 & 76 & D458 & 72 & 76 \\
& & & D459 & 72 & 76 \\
\hline
\end{tabular}




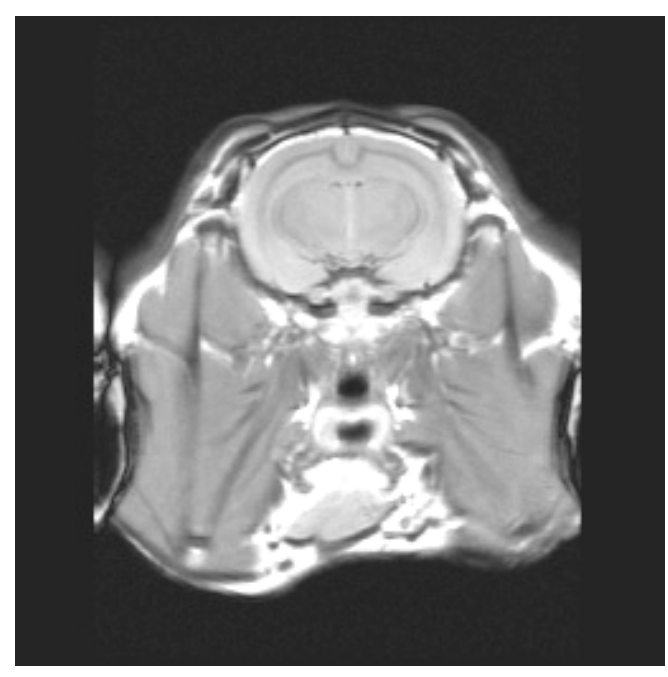

a) D443 FSE Image

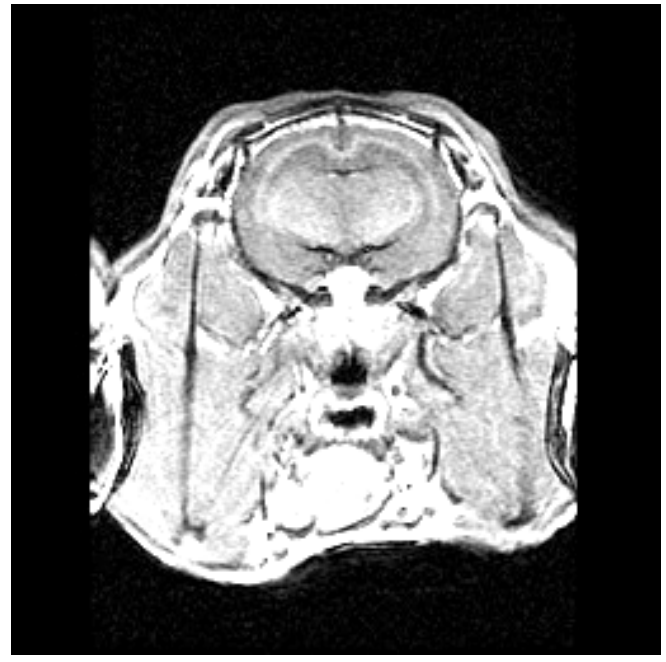

b) D443 FSPGR Image

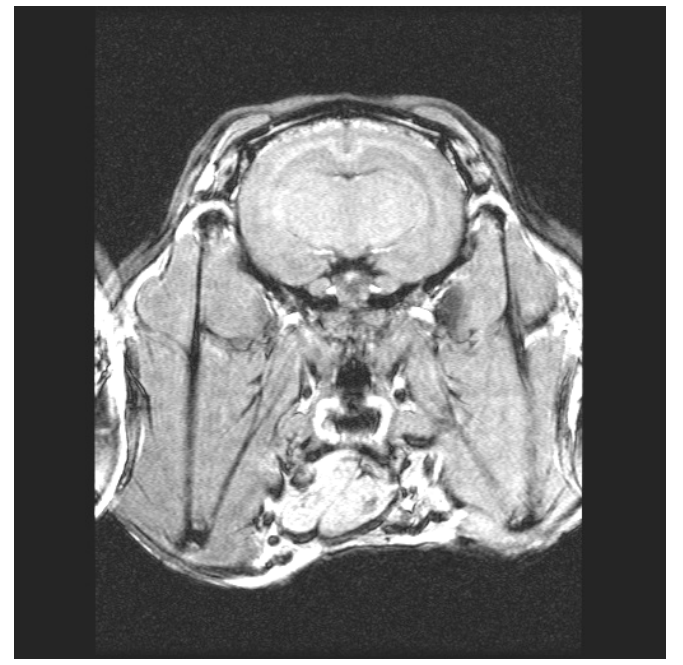

c) D443 TOF image

Figure 3.1: D443 Images of each modality 


\subsection{SLICE SELECTION}

To measure the ventricular volumes, it was necessary to segment the images of the brain that included the ventricles of interest. The first slice used in the automated analysis marks the beginning of the hippocampus in the frontal side. The third ventricle and lateral ventricles are hardly seen here. The subsequent slices gradually exhibit larger hippocampi, and show the appearance of the lateral and the third ventricles. As the slices continue further, the hippocampus and the ventricles start disappearing. Hence, the last slice chosen to be included in the analysis is the slice where the third ventricle disappears completely. Figure 3.2 shows the front end and back end of the rabbit brain. The alternate selected slices from animal D443 is shown in Figure 3.3.

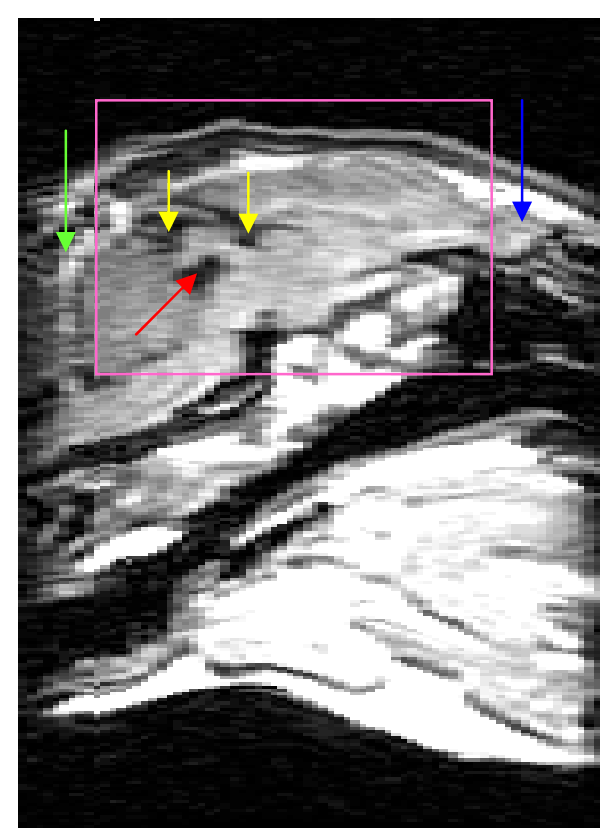

Figure 3.2: D443 FSPGR sagittal view

Front end (blue) and black end (green) of the brain. Location of brain (pink), third ventricle (red) and lateral ventricles (yellow) 


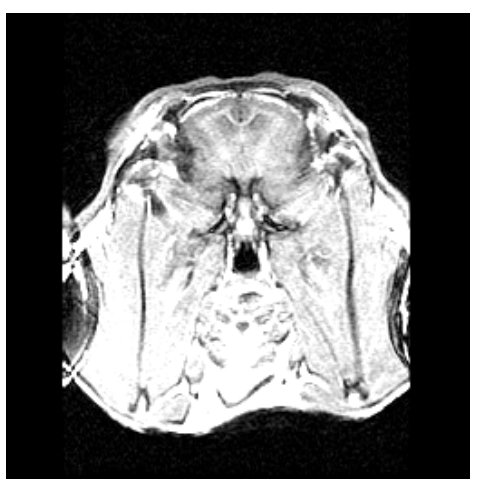

(a) no ventricles seen - front end

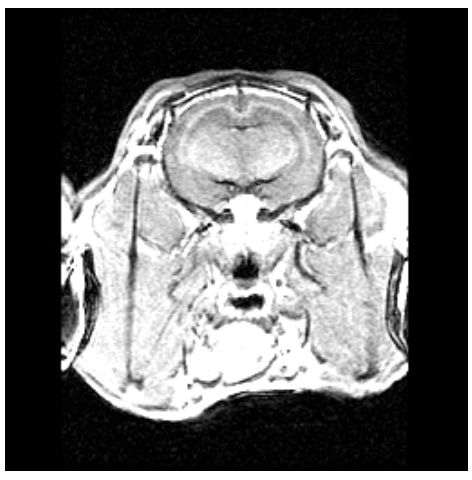

(d)

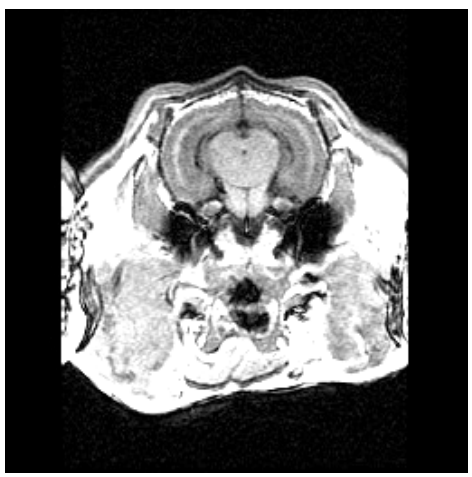

$(\mathrm{g})$

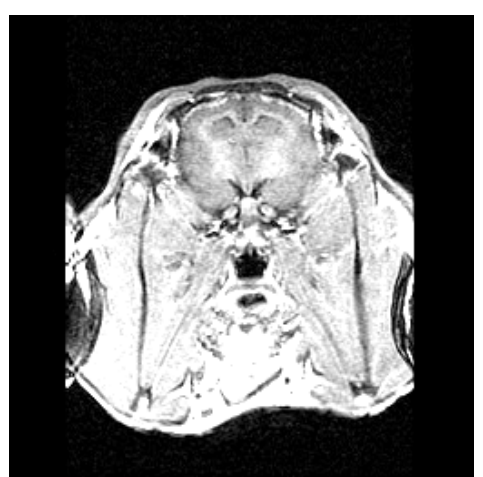

(b) start of third ventricle

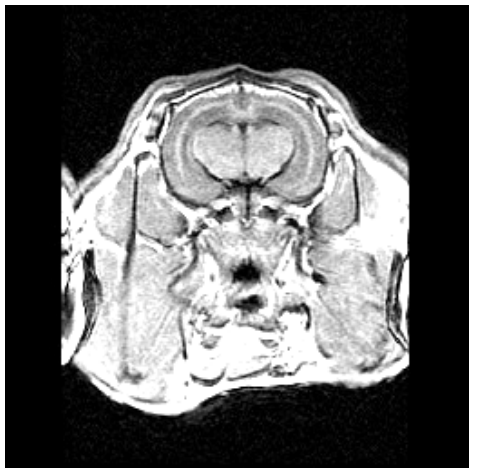

(e)

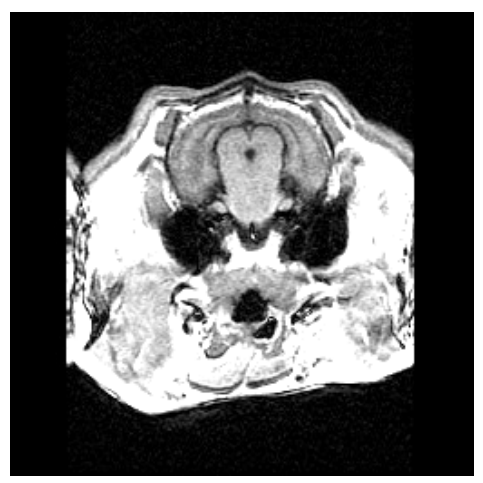

(h)

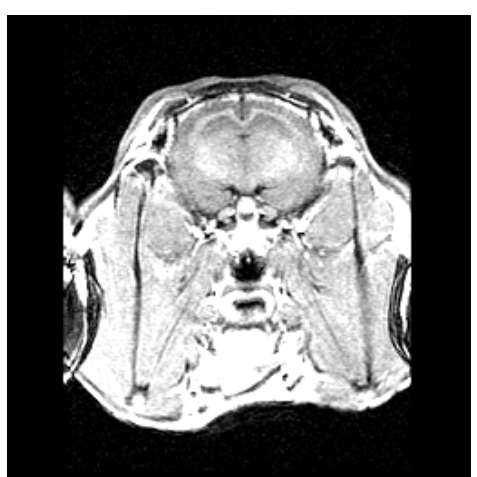

(c) $3^{\text {rd }}$ ventricle enlarges

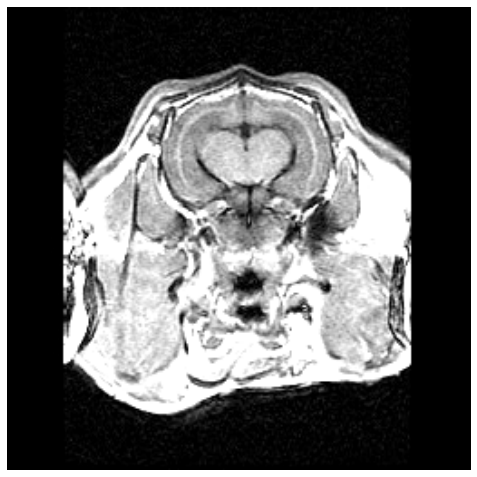

(f)

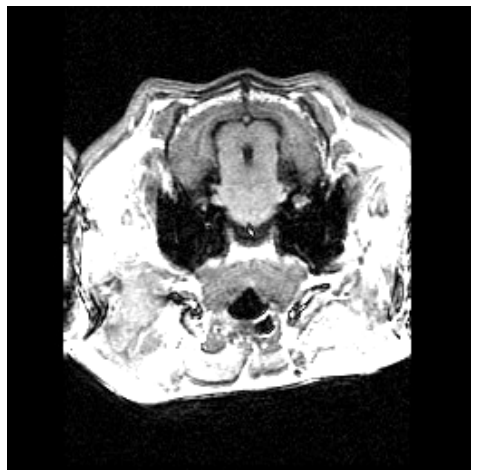

(i)

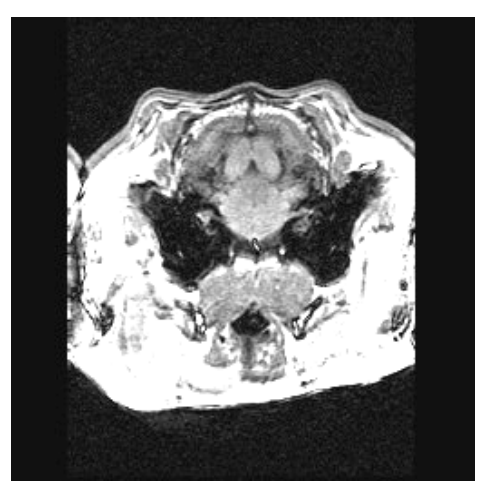

(j)

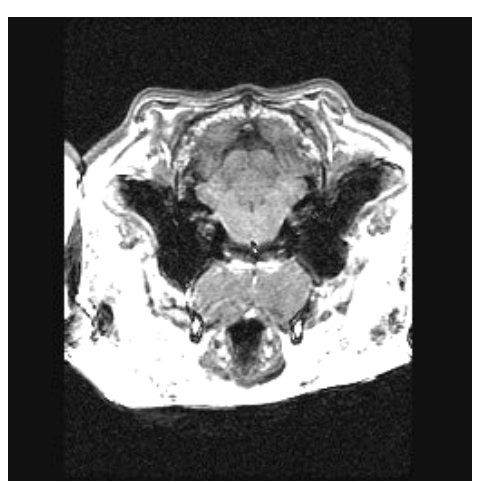

(k) no third ventricle seen - back end

Figure 3.3: Alternate slices of D443 FSPGR Images 


\subsection{HISTOLOGICAL DATA ANALYSIS}

A 3D high-resolution digital atlas of the rabbit brain has been constructed by Michael Gabriel and his associates in the Neuronal Patterns Analysis group at the Beckman Institute, University of Illinois, and Urbana Champaign. The histology rabbit brain atlas consists of 469 digital coronal images. A male White New Zealand rabbit was used for this purpose. A cryostat was used to slice the tissue and the sections were mounted on slides and scanned at a high resolution while still wet. A total of 469, 40micron coronal sections of a rabbit brain were digitized, aligned and conditioned using Analyze, Photoshop and custom techniques. Data sets were then transformed into 3D volumes and made available to users through a Java-based graphical front-end. Histological images with ventricles only were used for this work as shown in Figure 3.4 below.

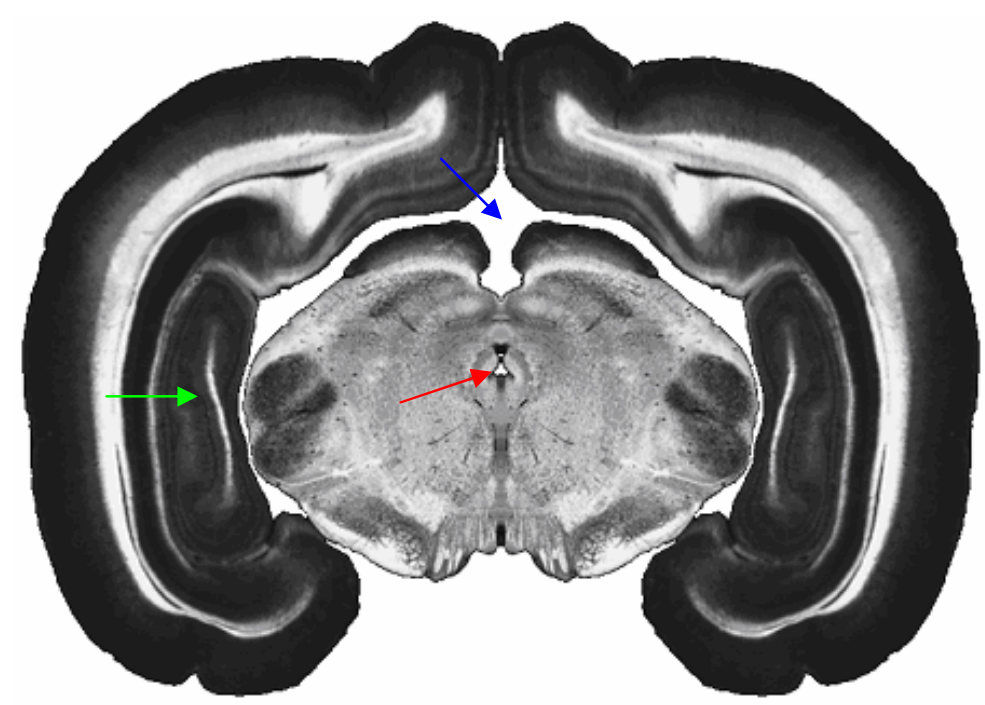

Figure 3.4: View of coronal digital histology image

Hippocampus (green), lateral ventricles (blue) and beginning of third ventricle (red) 


\subsection{STAGE 1: PERFORMANCE TEST OF GABOR WAVELETS ON RABBIT BRAIN MRI}

It was necessary to study the behavior of the Gabor wavelets on the different brain tissues as seen on MRI to verify if this method would be feasible in the extraction of the ventricles. Hence Stage 1 focused on the performance analysis of the Gabor wavelets on rabbit brain MRI. The Gabor filtering operation was performed on the FSE, FSPGR and TOF images to analyze the results on the different brain tissues in each modality. A "grey matter (GM)" and "white matter (WM)" block of size 8-by-8 pixels was chosen in the same region across the three modalities. The images showed in Figure 3.5 and 3.6 were used here.

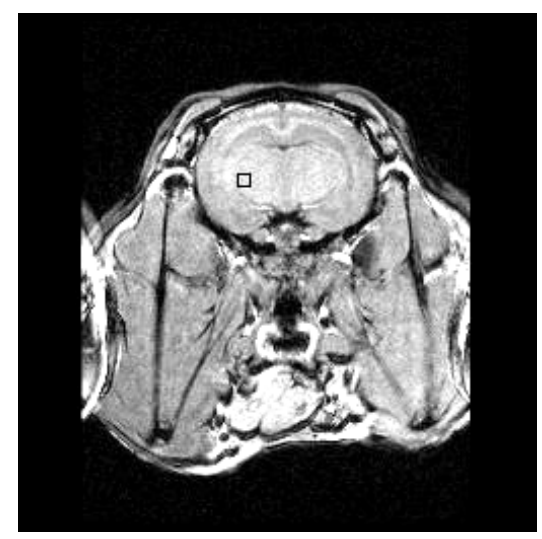

Figure 3.5: D443 TOF Image: 8-by-8 pixel WM block WM block (Black Square)

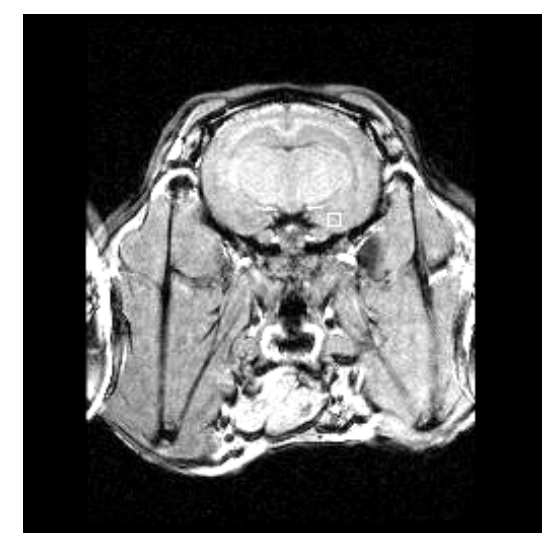

Figure 3.6: D443 TOF Image: 8-by-8 pixel GM block

GM block (White Square) 
We use $\eta_{s}=4$ and $\eta_{d}=8$ to extract texture features. We let the number of orientations be 8 to obtain the resolution information in the 8 directions. The number of scales was fixed based on the dilation of the image. Increasing the number of scales causes more dilation. Hence the number of scales was limited to 4. The product of $\eta_{s}$ and $\eta_{d}$ is the total number of filters that are being used for feature extraction.

The selection of appropriate window size was critical since smaller window size would lead to information loss and larger window size would include unwanted information too. In order to obtain the window size for this study, lateral ventricle widths were measured manually from the resized histological images and rabbit brain MRI. The average width from histological images was approximately 4 pixels and 2 pixels from the rabbit brain MRI as observed from Figure 3.7. Hence the window size of the filter was selected to be 3 .

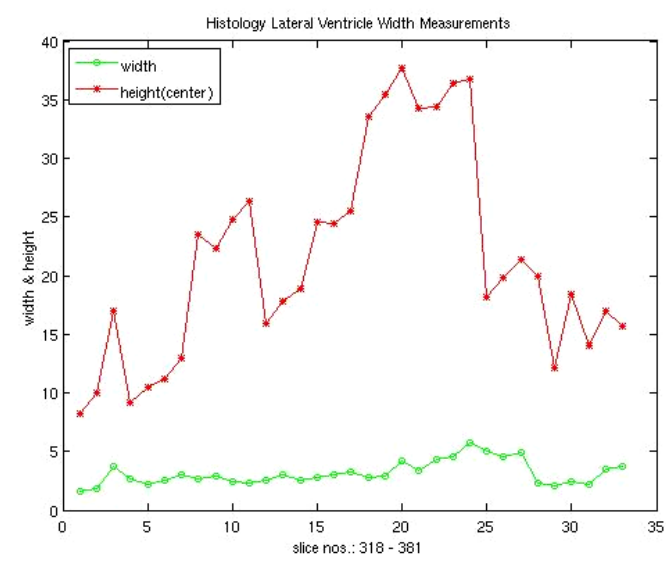

a) Resized histology

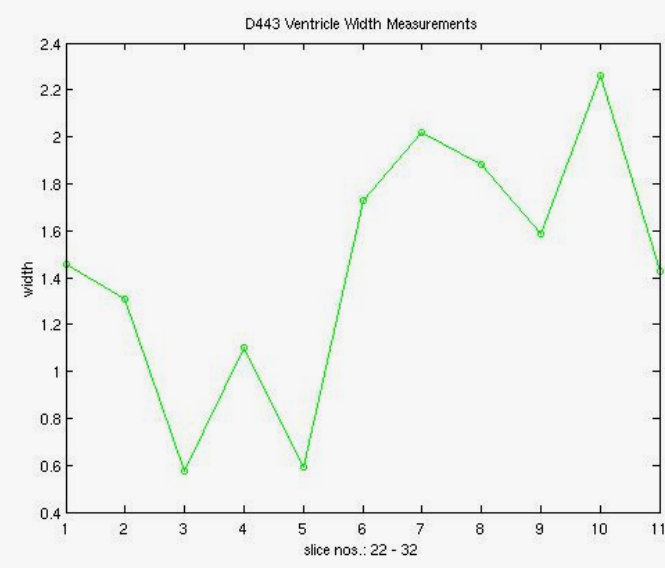

b) Rabbit brain MRI

Figure 3.7: Ventricle width measurements

The transformation produced by the Gabor wavelets can be represented by equation (2-5),

$$
E_{m n}(x, y)=\sum_{k} \sum_{l} I(x, y) g_{m n}^{*}(x-k, y-l)
$$


where $k, l$ are filter mask variables and $m, n$ are scale and orientation parameters, respectively. The function $g_{m n}^{*}$ is the complex conjugate of a two dimensional Gabor function with the regular grid $N \times N$. The preliminary tests conducted using Gabor wavelets on rabbit brain are explained in the next sections.

\subsubsection{Method I}

The total number of Gabor features obtained for an image depends upon the choice of scale and orientation parameters. Considering the total number of filters as the third dimension, a matrix comprising the Gabor features was formed. Hence, information for the same pixel from different filters is obtained. Similarity between gray matter and white matter tissues were evaluated by Euclidean distance measures.

The Euclidean distance is the straight line distance between two points i.e., the root of square differences between coordinates of pair of objects. In a plane with $p_{1}$ at $\left(x_{1}, y_{1}\right)$ and $p_{2}$ at $\left(x_{2}, y_{2}\right), D\left(p_{1}, p_{2}\right)$ is $\sqrt{\left(\left(x_{1}-x_{2}\right)^{2}+\left(y_{1}-y_{2}\right)^{2}\right)}$.

Firstly, the Euclidean distance was computed between one element of GM tissue and the other elements. This process was repeated for every element in the GM region. Secondly, the same procedure was repeated to all the elements of the WM region. Thus the variation measure within the same tissue type, also known as intra-class correlation variation was evaluated.

The inter-class correlation variation between the GM and WM was the next measure to be evaluated. It is defined as the variation measure between different classes. The distance between a GM element and each element of the WM region was obtained. This process was repeated for every element in the GM region. This process is equivalent to measuring the distance between a WM element and each GM element. All the parameters remained constant throughout the procedure. The plot of the Euclidean 
distance versus the different modalities for the three classes GM, WM and GM-WM is obtained.

\subsubsection{Method II (With adjustment of image intensity range)}

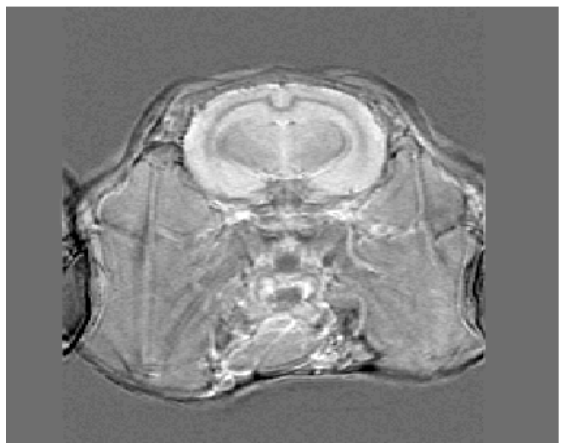

FSE FSPGR difference

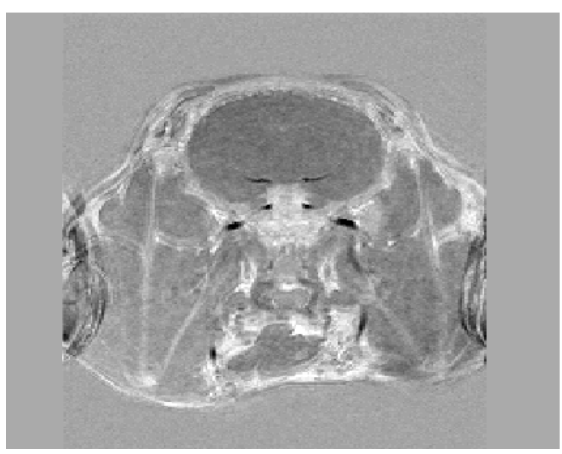

FSPGR TOF difference

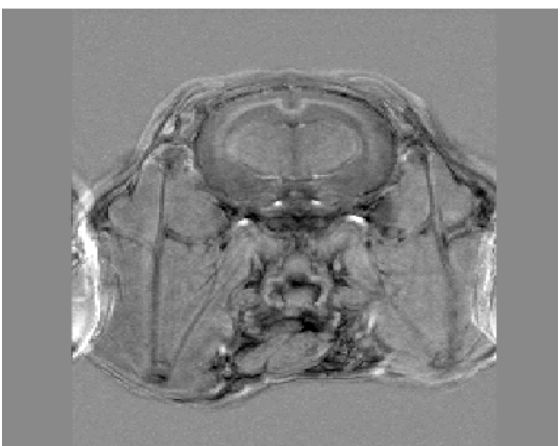

FSE TOF difference

Figure 3.8: Difference images

The intensities ranges of the three image modalities varied. Hence their intensities were rescaled in such a way that the minimum intensity value is 0 and the maximum value is 255 i.e. converted to 8 bit greyscale images. The difference images are obtained from the rescaled images, i.e., FSE-FSPGR, FSPGR-TOF and TOF-FSE as shown in Figure 3.8. The Gabor filtering operation was performed on the difference images. The intra-class correlation within the GM and WM was computed using the Euclidean distance measure. The inter-class correlation between the two tissues was computed using the Euclidean distance measure similar to the previous method. 


\subsection{STAGE 2: GABOR WAVELETS AND HISTOLOGICAL DATA}

The rabbit brain ventricles are small compared to the human brain. The images had low contrast, partial volume voxels (when the volume sampled by a single voxel contains more than one kind of tissue). Hence direct ventricle extraction was difficult. In order to the segment ventricles from the brain, it is necessary to segment the brain from the whole head rabbit MRI. Again, brain segmentation was not possible due to the unavailable rabbit brain atlas. Design of Gabor wavelet parameters for rabbit brain ventricle segmentation was also a challenging task.

One of the possible solutions was to design appropriate filters for segmenting ventricles from the histological rabbit brain images. Subsequently, the histology brain can be warped with the whole head rabbit MRI to extract the brain. The flowchart shown below in Figure 3.9 and 3.10 explains the work of Gabor wavelets on the histological data set.

Features in the flowchart have been detailed below:

- The histological images were 844 x 679 pixels in dimension. They had to be resized to the dimension of the MR images i.e. $256 \times 256$ pixels, so that the Gabor wavelet parameters used for the histology image could be used for further work on the rabbit brain MRI and for faster computation.

- One problem was that the intensity of the ventricles in the histological rabbit brain images was the same as that of the background. Hence the ventricle extraction was confounded by the background in the histological images. Manual selection of the brain region just including the ventricles helped in the segmentation problem.

- The seed point was chosen manually to segment the ventricles. It was selected from the third ventricle when it was seen clearly in the slice. Alternatively, it was selected at the point where the right and left lateral ventricles merged in slices where third ventricle was not clearly visualizable. In some of the slices, the third ventricle could not be seen clearly and it appeared very small in other slices. 


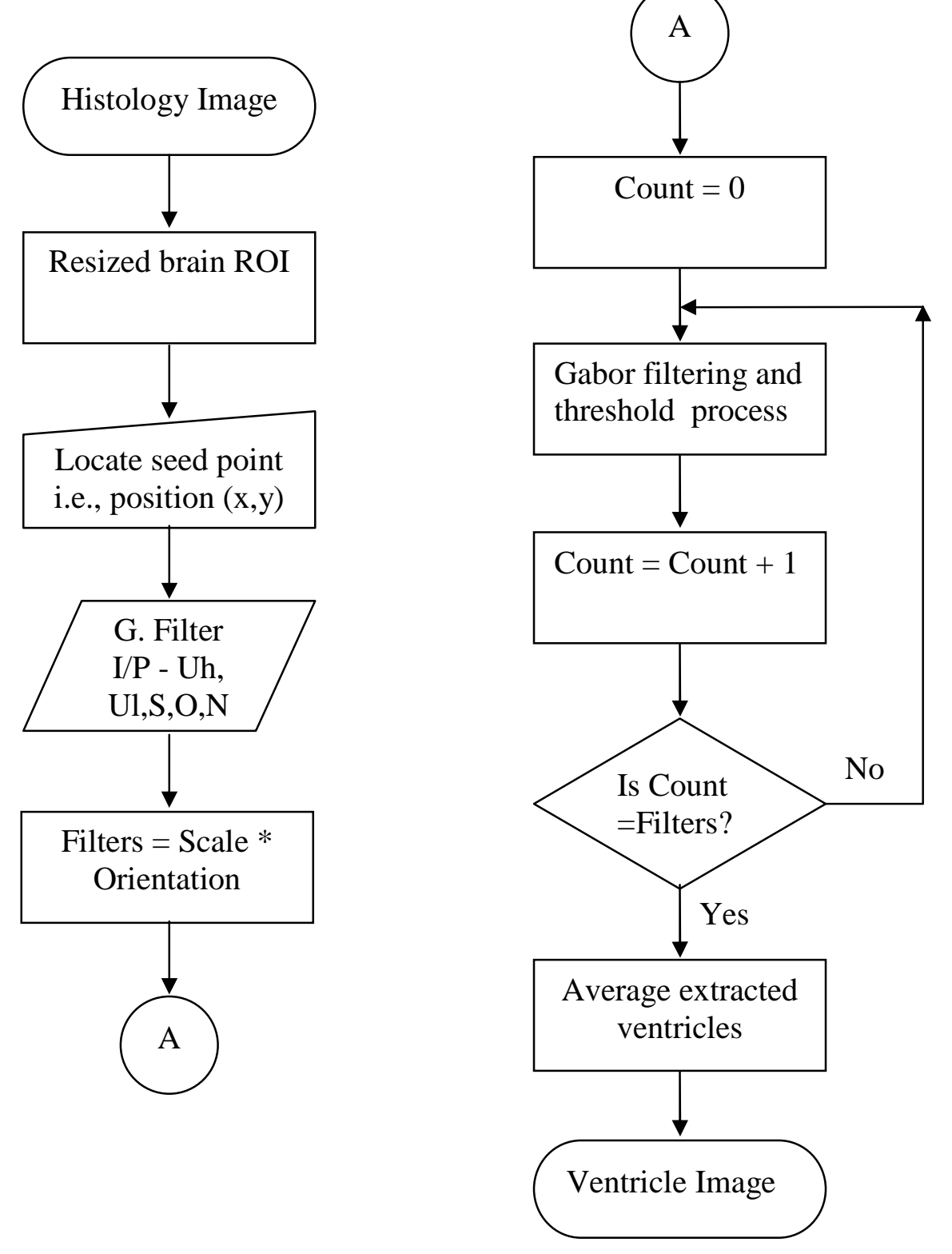

Figure 3.9: Flowchart for ventricle extraction process in histological images 


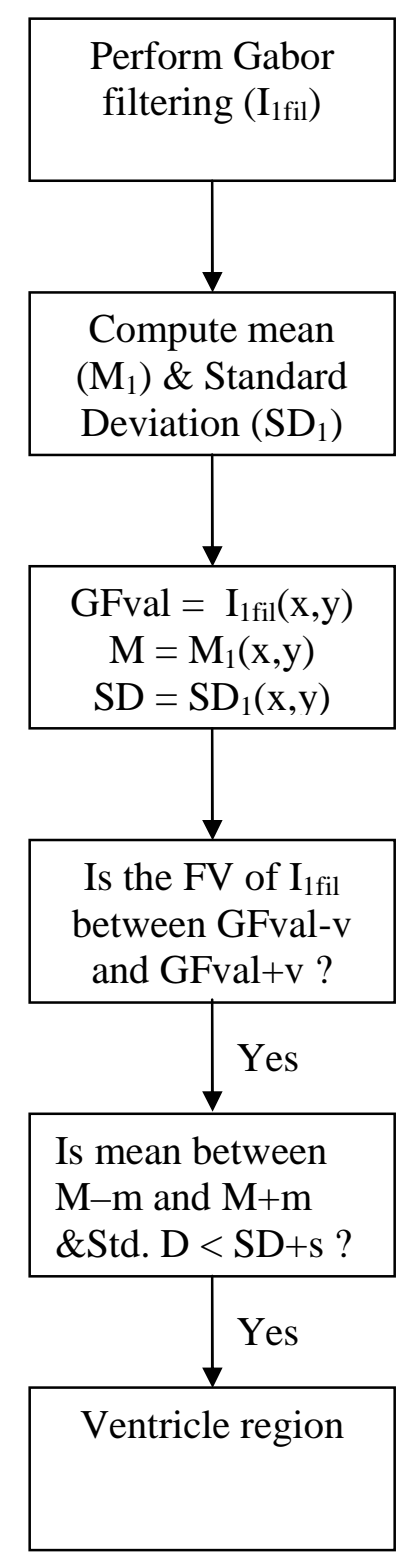

Figure 3.10: Gabor filtering and thresholding process 
- Preliminary studies of the Gabor wavelet parameter choices included the analyses for the choice of window size, upper and lower frequencies, scale and orientation. The parameters chosen for the performance test in Stage 1 was tried initially on the histological images.

- The product of scale and orientation was the total number of filters.

- Gabor filtering was performed on just the brain ROI which included ventricles only.

- The statistical measures (mean and standard deviation) were computed from the extracted feature.

- Thresholding based on the feature value of the seed point from Gabor response and statistical measures yielded a partial segmentation of the ventricles.

- The above three steps were repeated for the total number of filters.

- Average of all the partial segmentations of the ventricles obtained from each filter gave the final ventricle region for the given slice.

\subsection{STAGE 3: GABOR WAVELETS OF RABBIT BRAIN MRI}

\subsubsection{Manually Guided Segmentation Process}

This section focuses on the process of ventricle extraction from whole head rabbit MRI. The FSE T2 images could not be used because of the high slice thickness. They did not have enough slices discriminating ventricles from the rest of the brain tissues. This can be observed from Figure 3.11. The texture feature transformation is obtained from one modality at a time. The filtering and segmentation process are performed slice-byslice. The ventricle extraction process continued to be the same as Stage 2 except for one step. The native MR image dimension was 256 x 256 pixels. Hence image resizing was not necessary. Only the image intensity had to be rescaled to values occurring between 0 and 255 . 


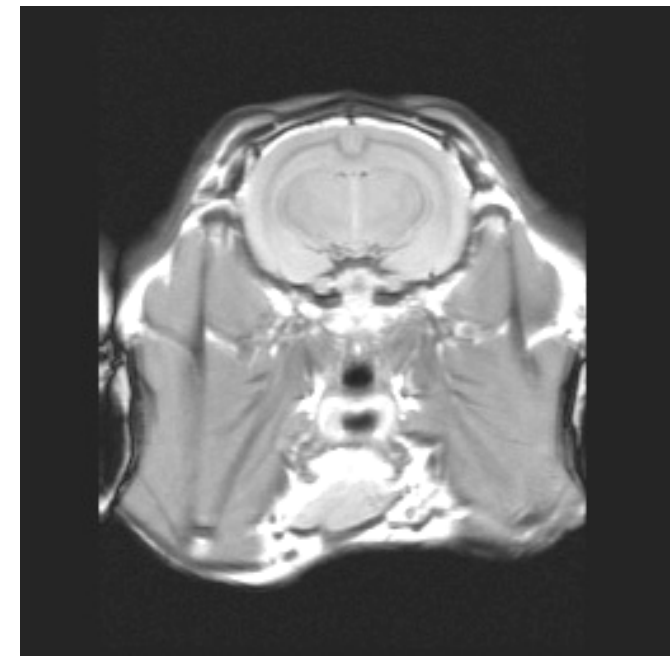

a) D443 FSE Image

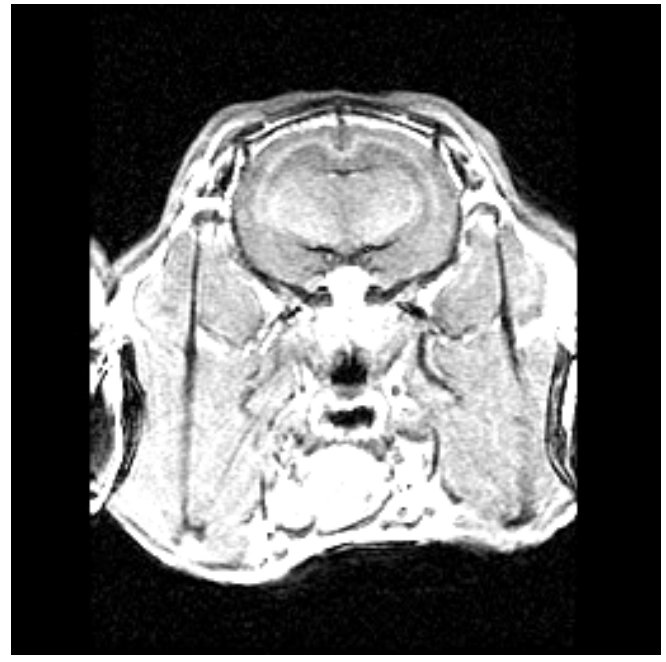

b) D443 FSPGR Image

Figure 3.11: D443 Images

\subsubsection{Automated Ventricle Segmentation Process}

It was necessary to automate the ventricle extraction process as manual extraction is tedious and time consuming. It can be observed from the ventricle extraction process on histological images that drawing of brain ROI with ventricles and seed point selection was performed manually. In order to automate the seed point selection, the region to select the seed point also needed to be automated. The automated work involves drawing seed point region and drawing brain ROI with ventricles

\subsubsection{Drawing Seed Point Region}

The landmark used to locate the center for drawing the region to locate the seed point was the peak present at the center of the skull. It lies almost midway in the image. The seed point was selected from the third ventricle or lateral ventricles. The center of the third ventricles if present was also along the midway few pixels (or rows) below the peak. Otherwise, it was selected from the hemisphere where the right and left lateral ventricles joins. The location of lateral and/or third ventricle was well along the midway following the peak on the skull. Hence detection of that peak was important. These features are shown in Figure 3.13. 


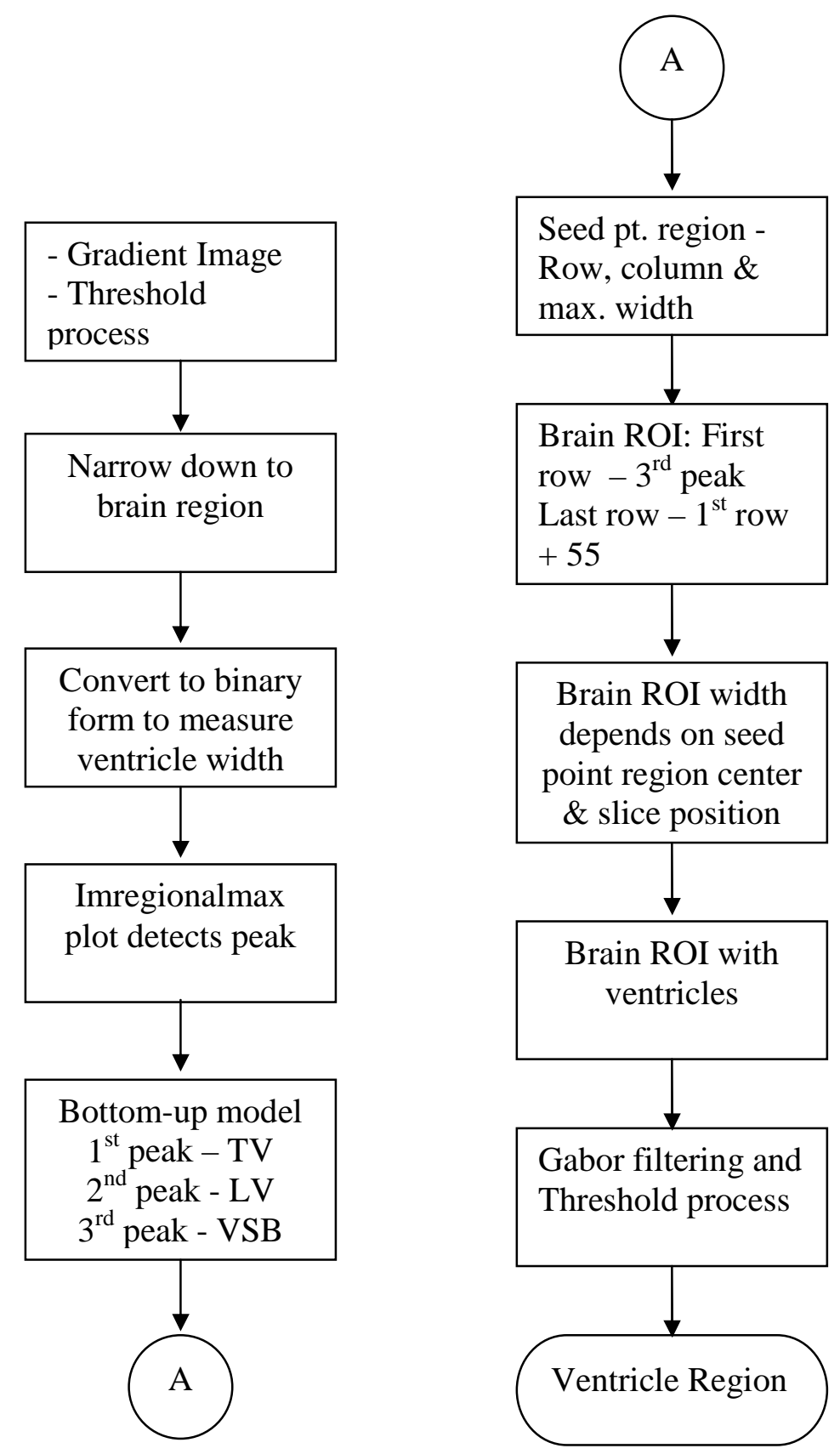

Figure 3.12: Flowchart of automated ventricle extraction process from whole head rabbit MRI

$\mathrm{TV}=$ Third ventricle, $\mathrm{LV}=$ Lateral ventricle and $\mathrm{CSF}=\mathrm{CSF}$ filled space between skull and brain 


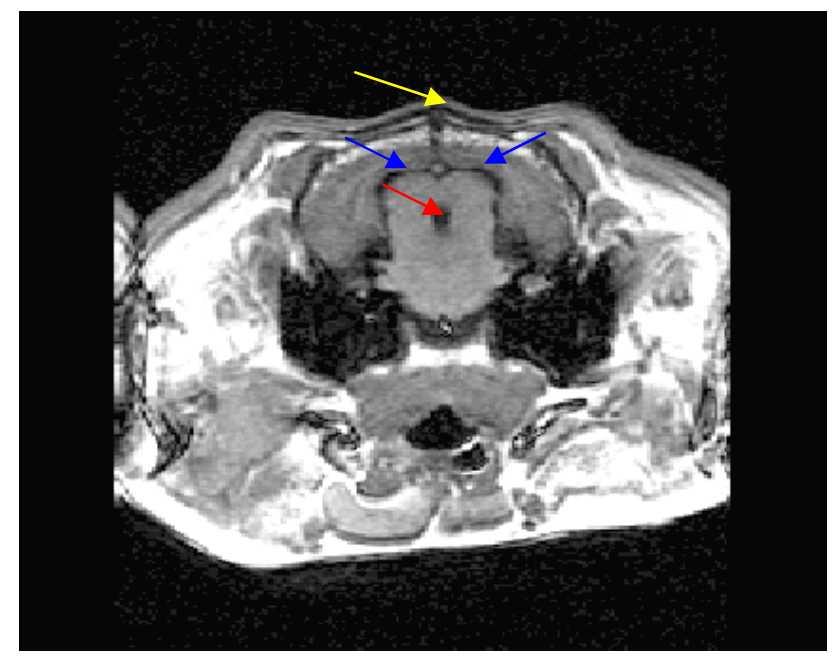

Figure 3.13: D443 FSPGR Image

Peak (yellow), lateral ventricle (blue), third ventricle (red)

\section{The following points explain the automated procedure:}

- The peak can be located once the edges of the image are obtained. However, there was noise in the background. This unwanted information was present above the peak on the skull. Figure 3.12 summarizes the automated procedure. The resulting output image at each stage is shown subsequently.

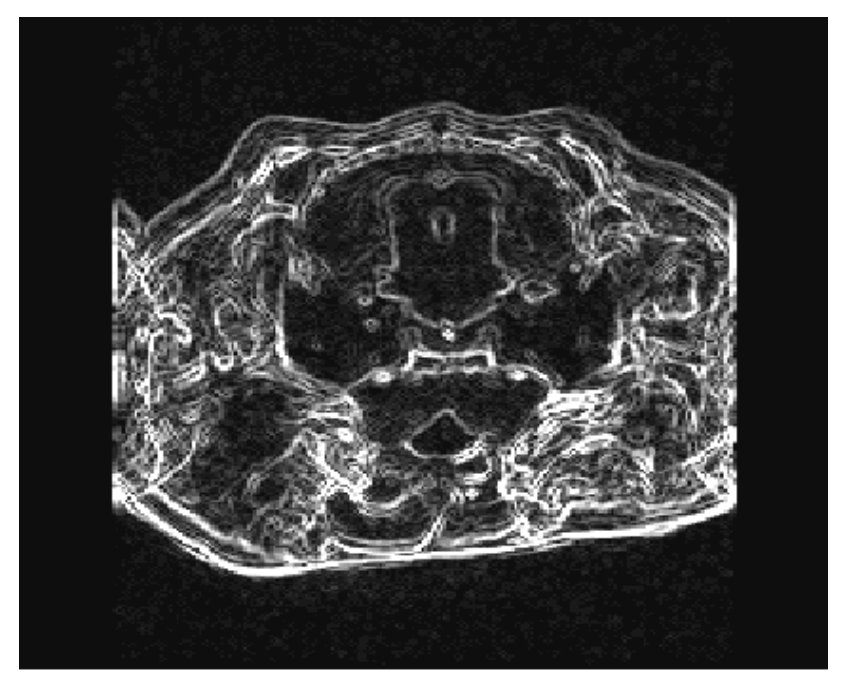

Figure 3.14: Gradient image 
- Edge extraction was performed by applying gradient filtering along the $\mathrm{x}$ and $\mathrm{y}$ directions.

- Threshold operation removes the noise in the background. The pixels with value ranging from 0 to 75 were removed. Thus noise in the background was eliminated.

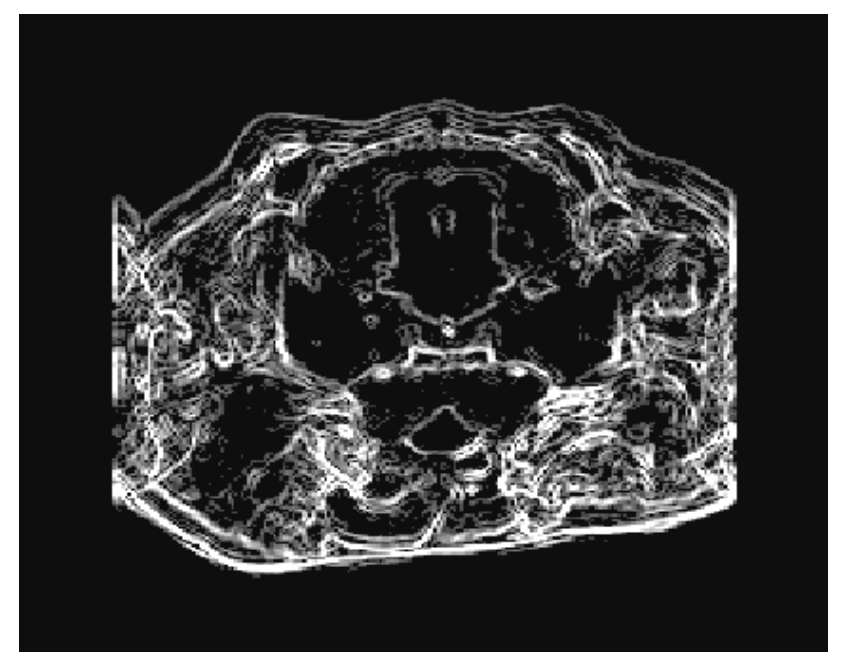

Figure 3.15: Thresholded gradient image

- It was observed from the whole head rabbit MR images that the peak was within the first half of the image. It was also noticed that the peak lay almost midway in the image. Sometimes, it was few pixels away from the midline either towards the right or left side of the given image.

- The next step was to narrow down to the region that would help in the peak detection. From the anatomy, it was observed that the peak was approximately within the first 100 rows (frow) from the image. It was also noticed that the peak could be located midway in the image. Hence, the peak was searched between columns 90 and 160 (fcol). Hence, the parameters of the region (block1) used for scanning the peak was:

$$
\begin{aligned}
& \text { Rows }(\text { frow })=1: 100 \\
& \text { Columns }(\text { fcol })=90: 160
\end{aligned}
$$




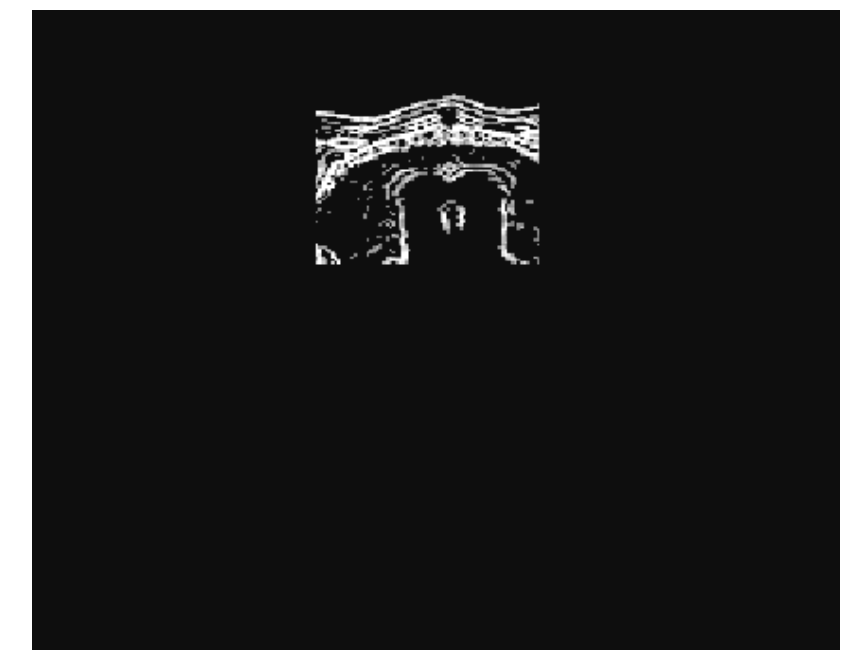

Figure 3.16: Reduced whole head rabbit MR image for peak detection

- From the above image, the peak can be identified easily. The first row containing the non-zero pixel gave the row information. Counting the number of non-zero pixels in the same row gave the total columns. Therefore, dividing the count by 2 gave the column information.

- The peak on the center of the skull was identified using the above explained process. The region of interest in this work was within the brain rather than the whole head rabbit MRI. Therefore, the next step was narrowing down brain region.

- It was also noticed from the images that the third ventricle if present was within the 60 rows from the peak, i.e., the length or height from the peak to the region below the third ventricle was 61 rows. This length included the lateral ventricles hemisphere too. The maximum width of the ventricles did not exceed beyond 15 pixels. Hence width of the brain was restricted to 12 columns on either directions of the column of the peak. Thus the brain region within the above mentioned rows and columns was taken for further study 


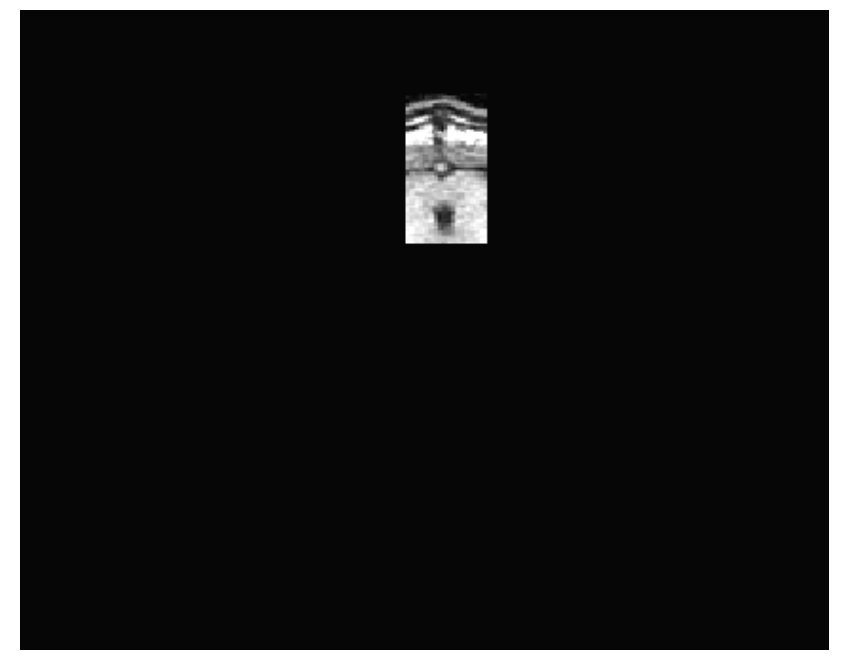

\section{Figure 3.17: Reduced brain region after peak detection}

- The ventricles appear dark in the above image. In order to extract information about ventricles location for seed point region drawing, the above image was converted to binary form using a threshold value of 225 . The binary image provided better view of the ventricles. It was noticed that the ventricle between the skull and the brain also appeared in the image.

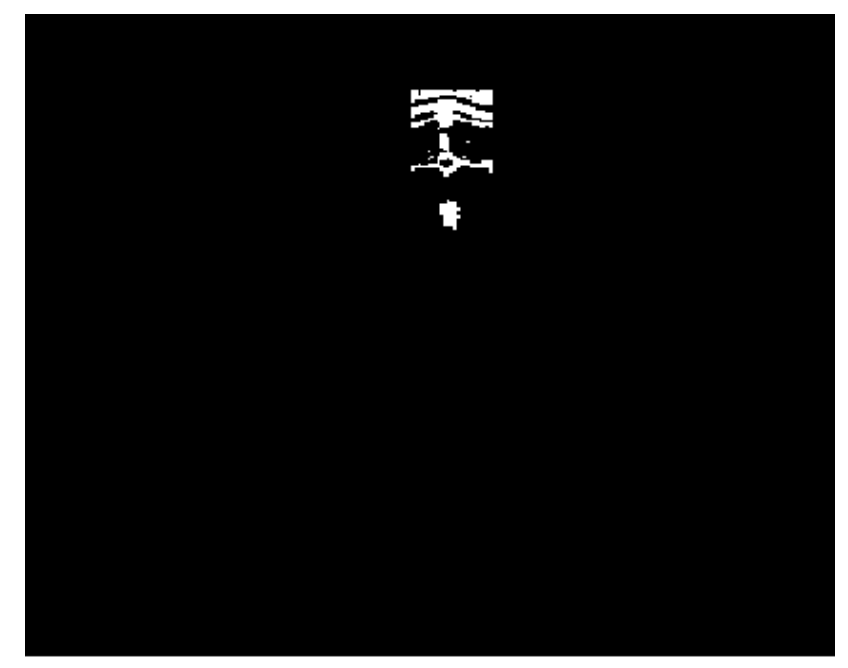

\section{Figure 3.18: Binary image to measure ventricles width}

- The ventricle between the skull and brain also appeared in the binary image. Thus the binary image has the lateral ventricles and third ventricle depending on the slice position. The ventricle between the skull and brain also interfered with the task of locating the ventricles. 
- The next step was to get closer to identify the seed point region using the peak location information. The brain area from the peak to just below the lateral ventricles or third ventricle was included. The area width was limited to include the maximum third ventricle knowledge only.

- The next stage of this study involved detecting the position to draw the region for seed point computation. Firstly, the width of the ventricles was measured along the $\mathrm{x}$ direction (widX) from the binary image. Now widX has the ventricles width along the $\mathrm{x}$ direction. On calculating its width vertically gave the height of the ventricles too (widXY). The plot widX showed peaks corresponding to the ventricles in the image.

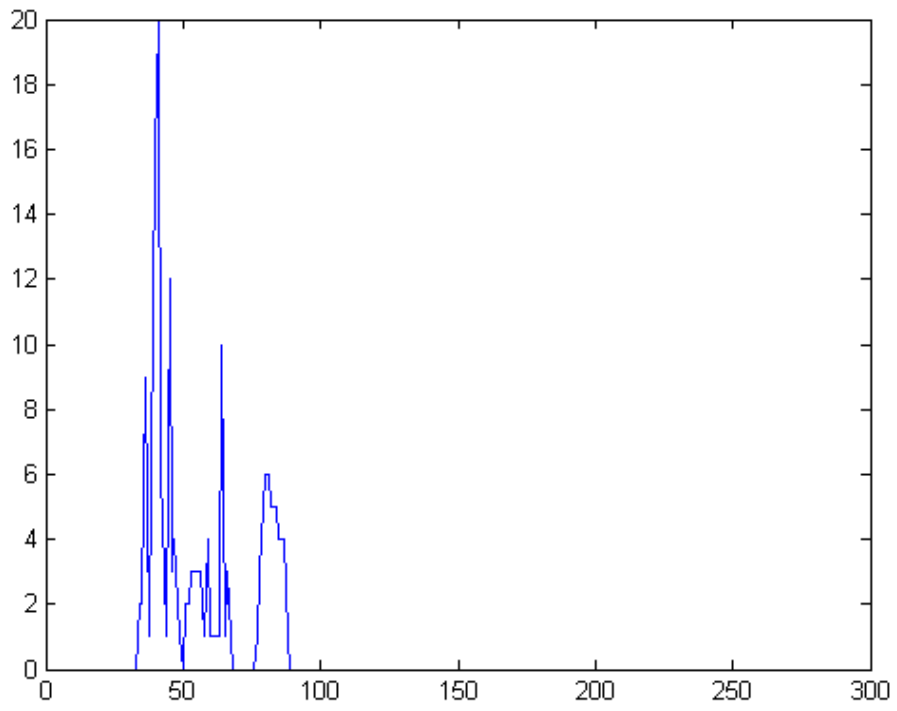

Figure 3.19: Ventricle width plot

- A MATLAB function - Imregionalmax was used to obtain the peaks. It finds the regional maxima of I and returns the binary image BW identifying the locations of the regional maxima in I. In BW, pixels set to 1 identify regional maxima; all other pixels are set to 0 . Regional maxima are connected components of pixels with the same intensity value $t$, and external boundary pixels all have a value less than $\mathrm{t}$. 


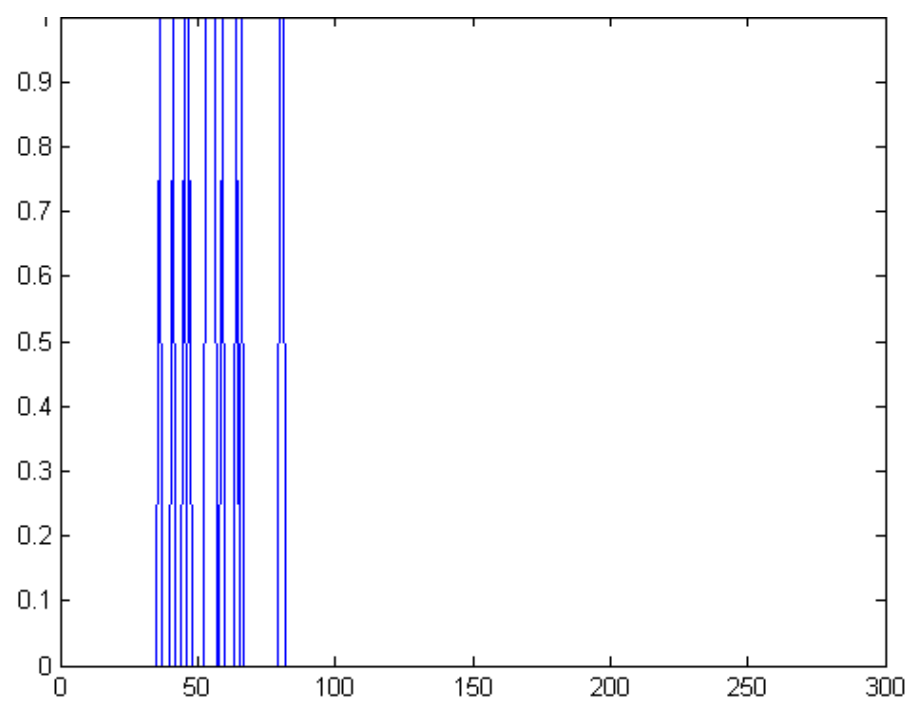

Figure 3.20: Imregionalmax function plot identifying peaks

- The plot of widX indicated the peaks of the ventricles at different regions. The number of peaks in the plot would be two or three depending upon the presence of the third ventricle. Extracting the information of the peak corresponding to third or lateral ventricle was difficult. Hence a bottom-up approach was followed. If the third ventricle was present, the last peak from the imregionalmax results corresponded to the third ventricle. Otherwise it was the lateral ventricle.

- Using the information from widXY and results of imregionalmax, the row (srow) position was determined. Hence srow and fcol was center point for drawing the block to select the seed point. The maximum width along fcol and srow helped determining the height and width of the block. The pixel with the lowest intensity was chosen as the seed point and its location was used in the intensity rescaled given image to extract ventricles 


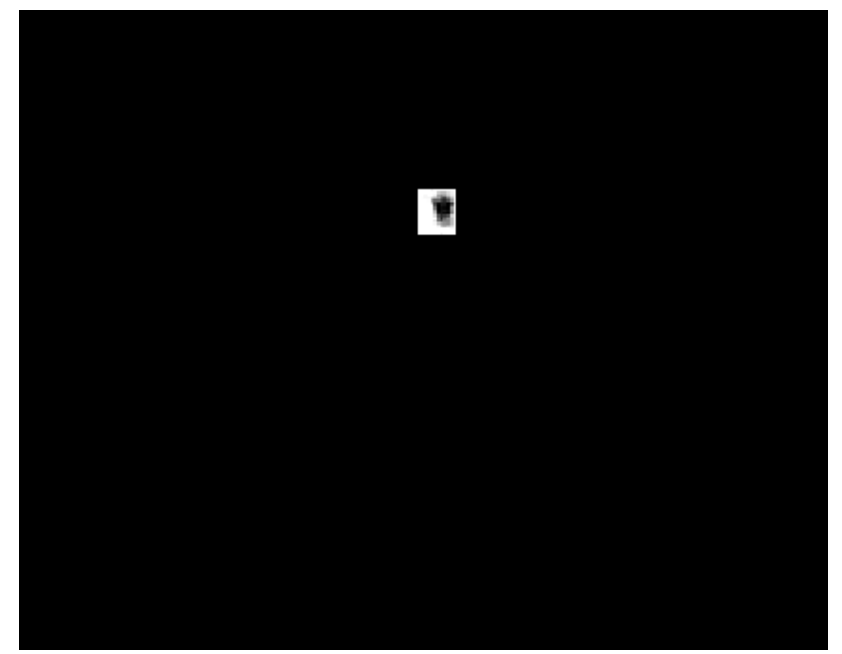

Figure 3.21: Automated seed point region selection

\subsubsection{Drawing the Brain ROI with Ventricles only}

- This process depends on the earlier seed point region drawing. Slices were taken as explained in the slice selection section. The fifth image of every set had a clear view of third ventricle and lateral ventricles. Firstly, this image was used to select the brain ROI with ventricles. The brain ROI for the other slices was drawn using the information obtained from this image.

- Brain region (B1) was restricted for the dimensions below:

- height $=30.03 \mathrm{~mm}$, i.e., rows $=$ srow $:$ srow +90 .

- $\quad$ width $=39.93 \mathrm{~mm}$, i.e., columns $=$ scol-60: $\mathrm{scol}+60$.

- B1 was converted into binary image using a threshold value of 225 .

- Information from widXY helped locating the first row of the brain ROI.

- Imregionalmax identified at least three peaks:

- third ventricle, lateral ventricle and CSF filled space between the skull and the brain.

- First peak identified the CSF filled space between the skull and the brain.

- The row corresponding to the first peak became the first row (r1) for brain ROI.

- From the anatomy, it was observed : 
- the ventricle was within $16.5 \mathrm{~mm}$ to $19.8 \mathrm{~mm}$ from the top position of the brain i.e., 50 to 60 rows from the first row.

- length of the brain with ventricles was $18.5 \mathrm{~mm}$, i.e., last row of the brain ROI was $\mathrm{r} 1+55$.

- Width of the brain ROI was determined with the information from seed point region center.

- It was observed from the anatomy, that the width was between $18.5 \mathrm{~mm}$ and $24.75 \mathrm{~mm}$ depending upon the slices.

- Thus brain ROI selection was automated and brain ROI was drawn. It can be seen in Figure 3.22.

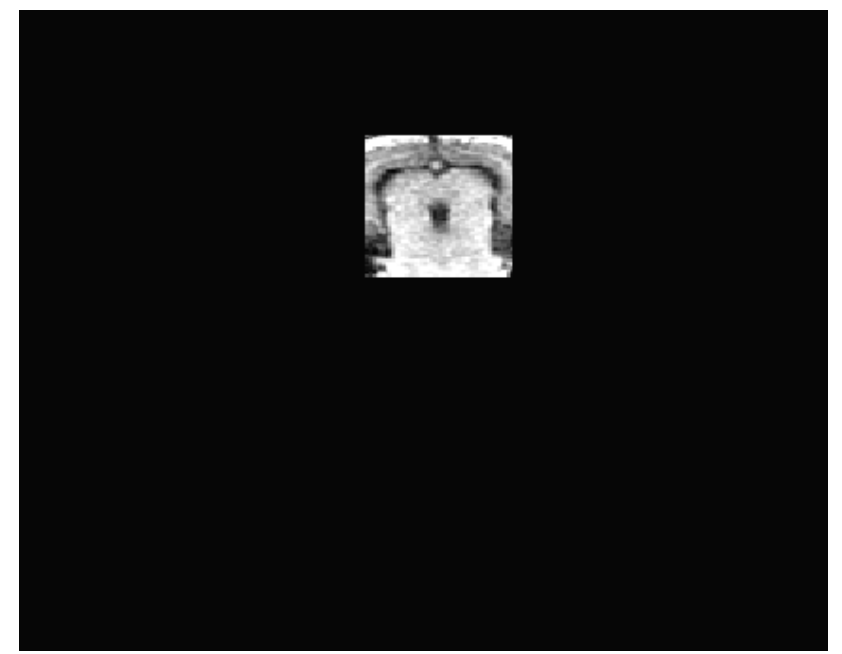

Figure 3.22: Automated selection of brain ROI with ventricles

\subsubsection{Locating the Seed Point}

The method of determining the seed point within the region obtained using the above process is explained in this section. Our objective was to identify the seed point, i.e., the pixel with the lowest intensity in the given region. Firstly, a horizontal scan was performed within the seed point region to obtain the pixel with lowest intensity value. Thus the minimum intensity value from each row was obtained. Secondly, the lowest 
value from the above result gave the seed point value and its corresponding location was the seed point location.

\subsubsection{Ventricle Extraction}

The seed point location was obtained from the above process. The ventricle extraction was done using the methods explained in the latter part of Stage 3. The mean and standard deviation was computed for each Gabor filter response. The threshold values based on the feature values of Gabor response mean and standard deviation aided in the ventricle extraction. This process for repeated for all the filters and the average of the ventricles yielded the ventricles for the given image. This process was repeated for every slice chosen for the corresponding animal. 


\section{CHAPTER 4: RESULTS}

In this chapter, the results of the studies at the three stages are discussed. The objective of this work was to examine if the texture features obtained using Gabor wavelets can help in the ventricle extraction. The simulations were carried out in a MATLAB 6.1 environment, using Dell Precision Workstation 650 with Intel Pentium(R) M processor, $2.4 \mathrm{GHz}$ and $1 \mathrm{~GB}$ RAM.

\subsection{STAGE 1: PERFORMANCE TEST OF GABOR WAVELETS ON RABBIT BRAIN MRI}

The performance tests were performed initially to check the performance of the Gabor wavelets on the different brain tissues GM and WM.

\subsubsection{Method I}

The Euclidean distance measure was used to observe the intra-class correlation within the GM and WM tissues. The inter-class correlation between GM and WM was also computed using Euclidean distance. The plot shows the results of both the correlation measures.

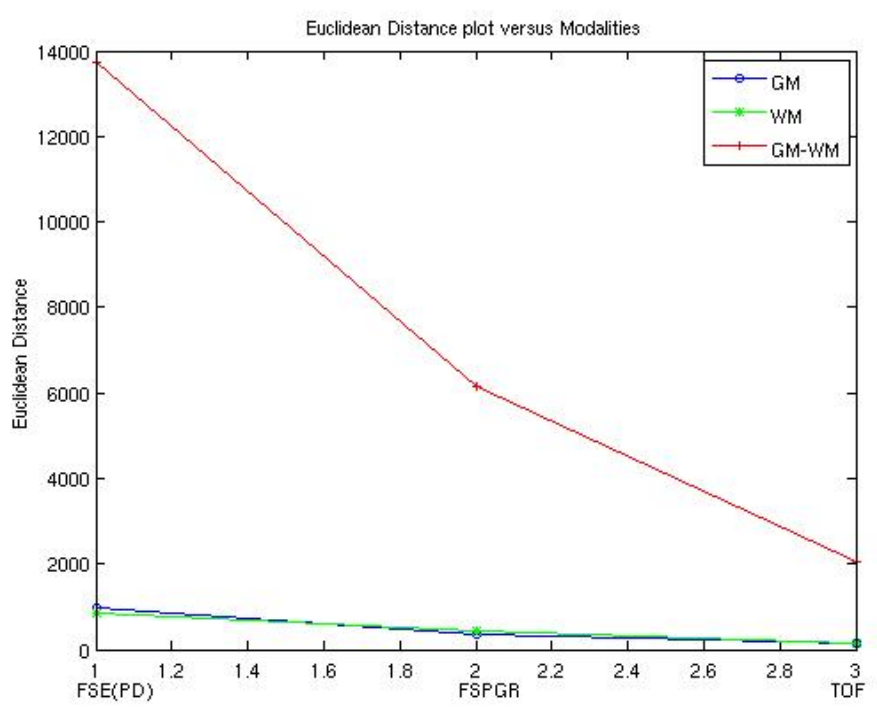

Figure 4.1: Euclidean distance plot versus modalities 
It can be observed from the Figure 4.1 that correlation within the GM elements appeared the same. Similar behavior was also observed with the WM tissue elements. And the intra-class correlation results were low. The inter-class correlation, i.e., the correlation between the GM and WM tissues were high compared to the intra-class correlation results. Thus, it was concluded that Gabor wavelets was able to discriminate the different tissue types.

\subsubsection{Method II}

The difference images from three modalities were obtained. The intra-class correlation and inter-class correlation measures are computed similar to the previous method.

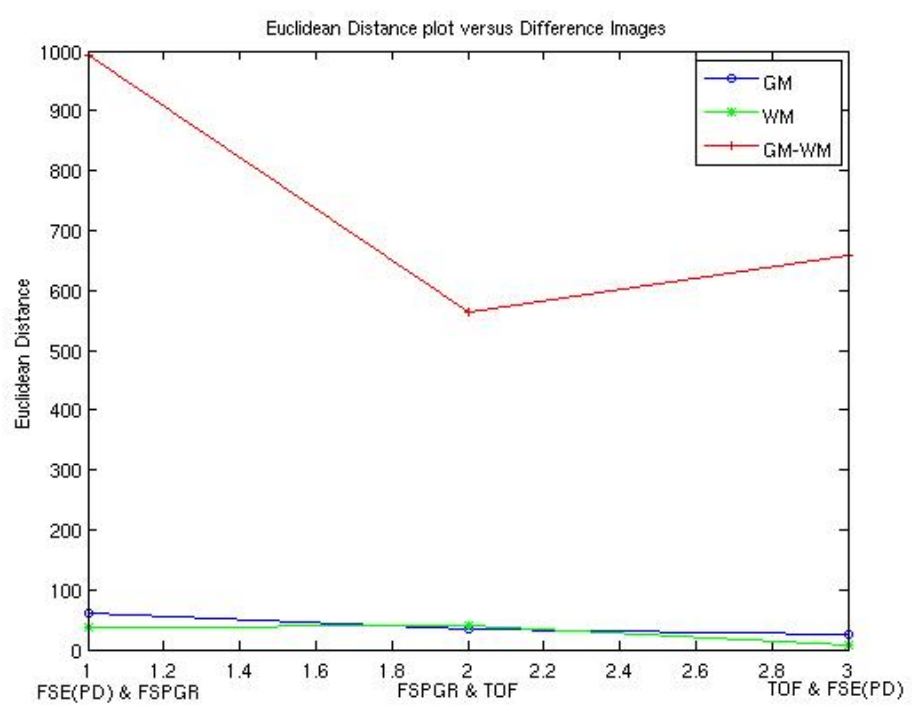

Figure 4.2: Euclidean distance plot versus difference images

From the Euclidean distance measures of difference images, it can be observed from Figure 4.2 that correlation within the GM elements appeared the same. Similar behavior was also observed with the WM tissue elements. And the intra-class correlation results were low. The inter-class correlation, i.e., the correlation between the GM and WM tissues were high compared to the intra-class correlation results. Thus from both the results, it can be clearly known that the Gabor wavelets was able to identify the different tissue types. 


\subsection{STAGE 2: GABOR WAVELETS ON HISTOLOGICAL IMAGES}

The performance results of the Gabor wavelets on the histology images are explained in this section. A region of interest was selected from the histology image as explained in the Stage 2 of Chapter 3.

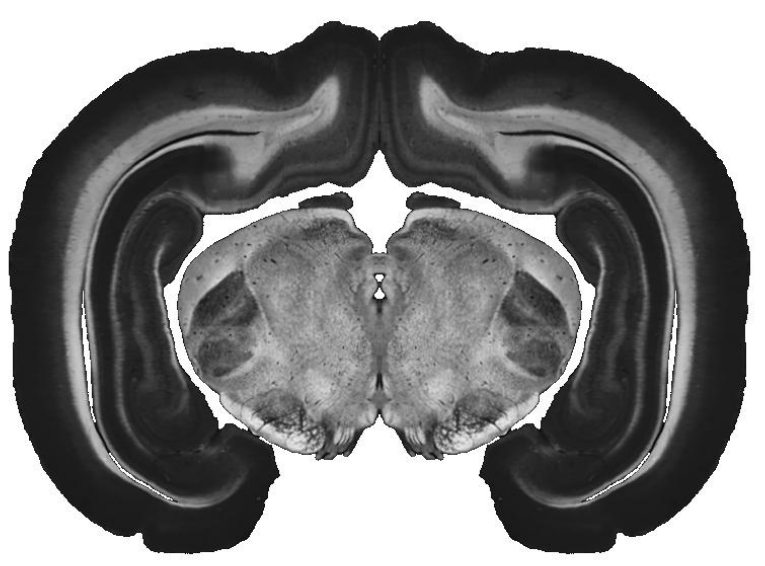

Figure 4.3: Histology image

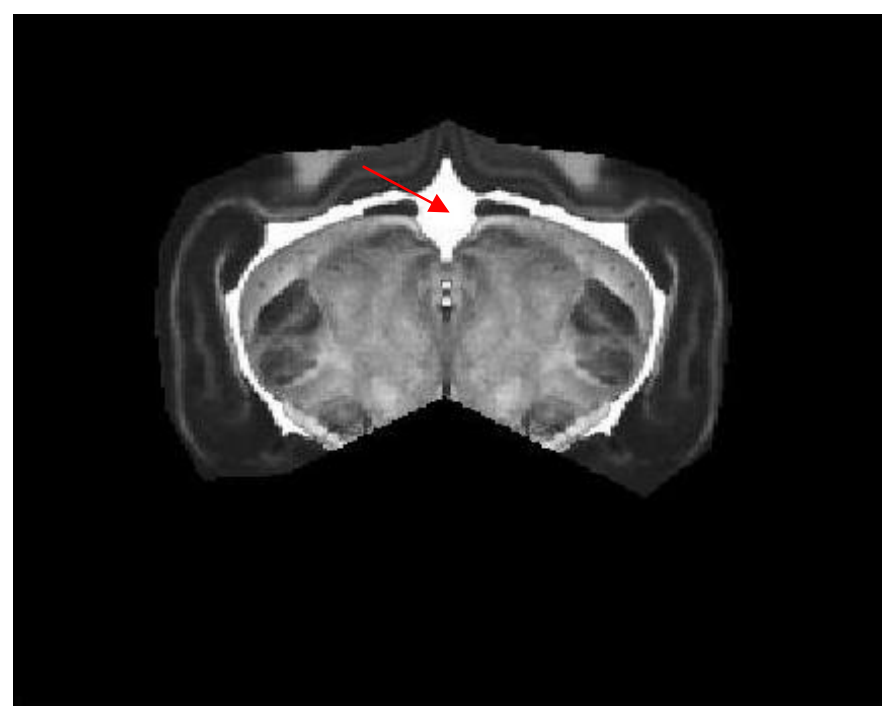

Figure 4.4: Brain ROI with ventricles and seed point selection (red) 
The parameters used for the Stage 2 analysis were used in this section. The Gabor parameters and the threshold values used for the ventricle extraction process on the histology images are shown in Table 4.1.

Table 4-1: Gabor filter and threshold parameters for histological images

\begin{tabular}{|c|c|}
\hline Parameters & Values \\
\hline Window size, $\mathrm{N}$ & 3 \\
Upper frequency, $\mathrm{U}_{\mathrm{h}}$ & 0.4 \\
Lower frequency, $\mathrm{U}_{\mathrm{l}}$ & 0.05 \\
Scale, $\mathrm{m}$ & 4 \\
Orientation, $\mathrm{n}$ & 8 \\
\hline Gabor response feature value based range & Gthresh-2 : Gthresh+2 \\
Mean threshold range & Mean_SdPt-7 : Mean_SdPt+7 \\
Standard Deviation threshold range & $<=\mathrm{SD}$ SSdPt+10 \\
\hline
\end{tabular}

Where,

Gthresh $=$ Feature value at the seed point location in the Gabor response

Mean_SdPt $=$ Mean value at the seed point location

SD_SdPt $=$ Standard Deviation at the seed point location

Figure 4.5 and 4.6 shows the results of the ventricle extraction in the histological image. The ventricles are colored and overlaid on the histological image. 

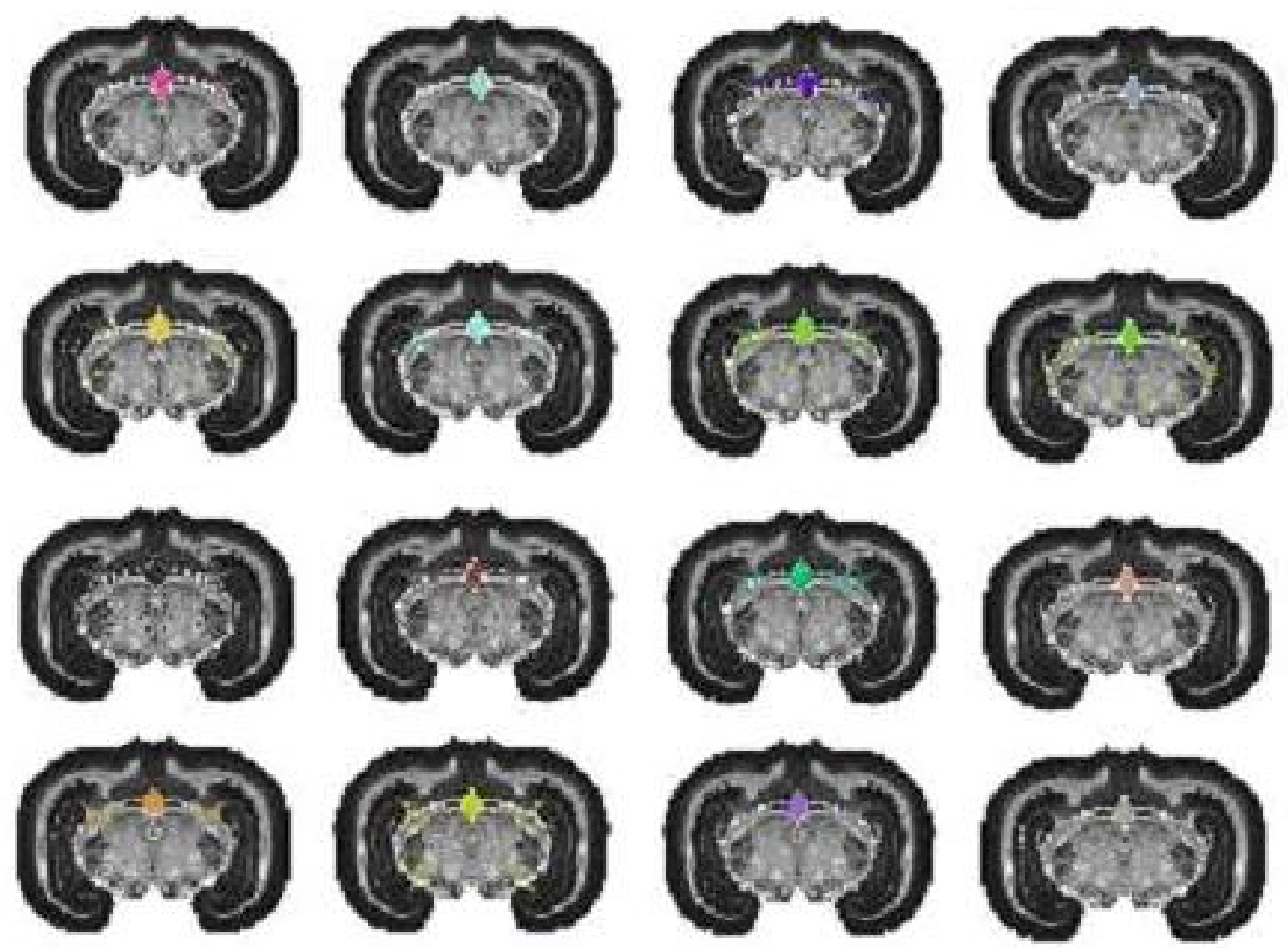

Figure 4.5: Ventricles overlaid on Histology Image

First row: scale $=1$ and orientations $=1$ to 4 (L to $R$ )

Second row: scale $=1$ and orientations $=5$ to 8 ( $\mathrm{L}$ to $\mathrm{R}$ )

Third row: scale $=2$ and orientations $=1$ to 4 (L to $\mathrm{R}$ )

Fourth row: scale $=2$ and orientations $=5$ to 8 (L to R) 


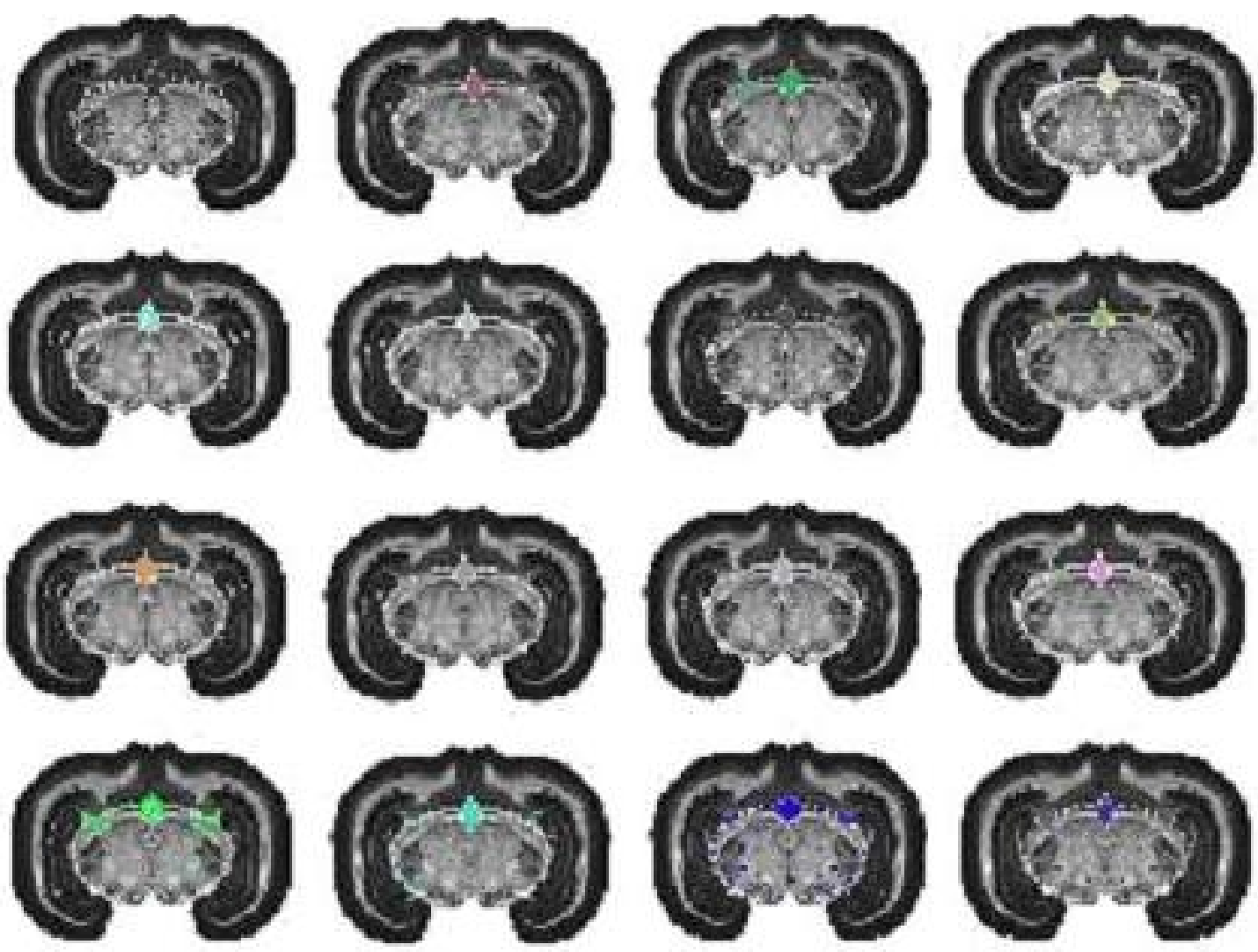

Figure 4.6: Ventricles overlaid on Histology Image

First row: scale $=3$ and orientations $=1$ to 4 (L to R)

Second row: scale $=3$ and orientations $=5$ to 8 (L to $\mathrm{R}$ )

Third row: scale $=4$ and orientations $=1$ to 4 (L to R)

Fourth row: scale $=4$ and orientations $=5$ to 8 ( $\mathrm{L}$ to $\mathrm{R}$ ) 


\subsection{STAGE 3: GABOR WAVELETS ON RABBIT BRAIN MRI}

\subsubsection{Manually Guided Segmentation Process}

The results of the Stage 3, manually guided segmentation process in FSPGR and TOF images are shown in Figure 4.7, 4.8, 4.9 and 4.10. The manual Brain ROI and seed point selection is also indicated.

Table 4-2: Gabor filter and threshold parameters for rabbit brain MRI

\begin{tabular}{|c|c|}
\hline Parameters & Values \\
\hline Window size, $\mathrm{N}$ & 3 \\
Upper frequency, $\mathrm{U}_{\mathrm{h}}$ & 0.4 \\
Lower frequency, $\mathrm{U}_{\mathrm{l}}$ & 0.05 \\
Scale, $\mathrm{m}$ & 2 \\
Orientation, $\mathrm{n}$ & 8 \\
\hline Gabor response feature value based range & Gthresh-15 : Gthresh+15 \\
Mean threshold range & Mean_SdPt-25 : Mean_SdPt+25 \\
Standard Deviation threshold range & $<=$ SD_SdPt+40 \\
\hline
\end{tabular}

The parameters mentioned in Table 4.2 were used for the manually guided segmentation. Figure 4.7 is the result obtained with manually drawn brain ROI and manually selected seed point within the brain ROI. The next Figure 4.8 selects the seed point automatically within the manually chosen seed point region within the brain ROI. 

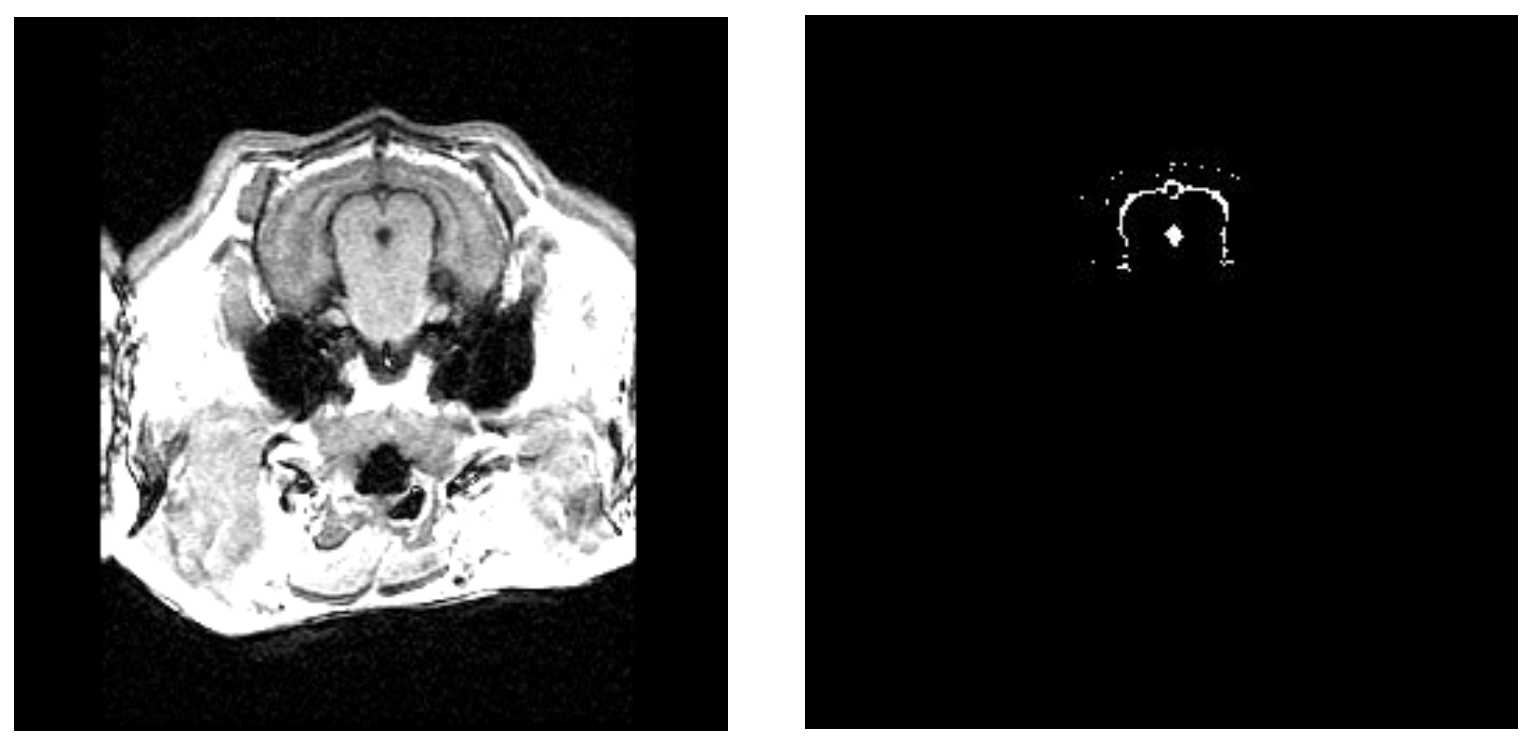

Figure 4.7: Manual seed point selection - D443 FSPGR Image 24
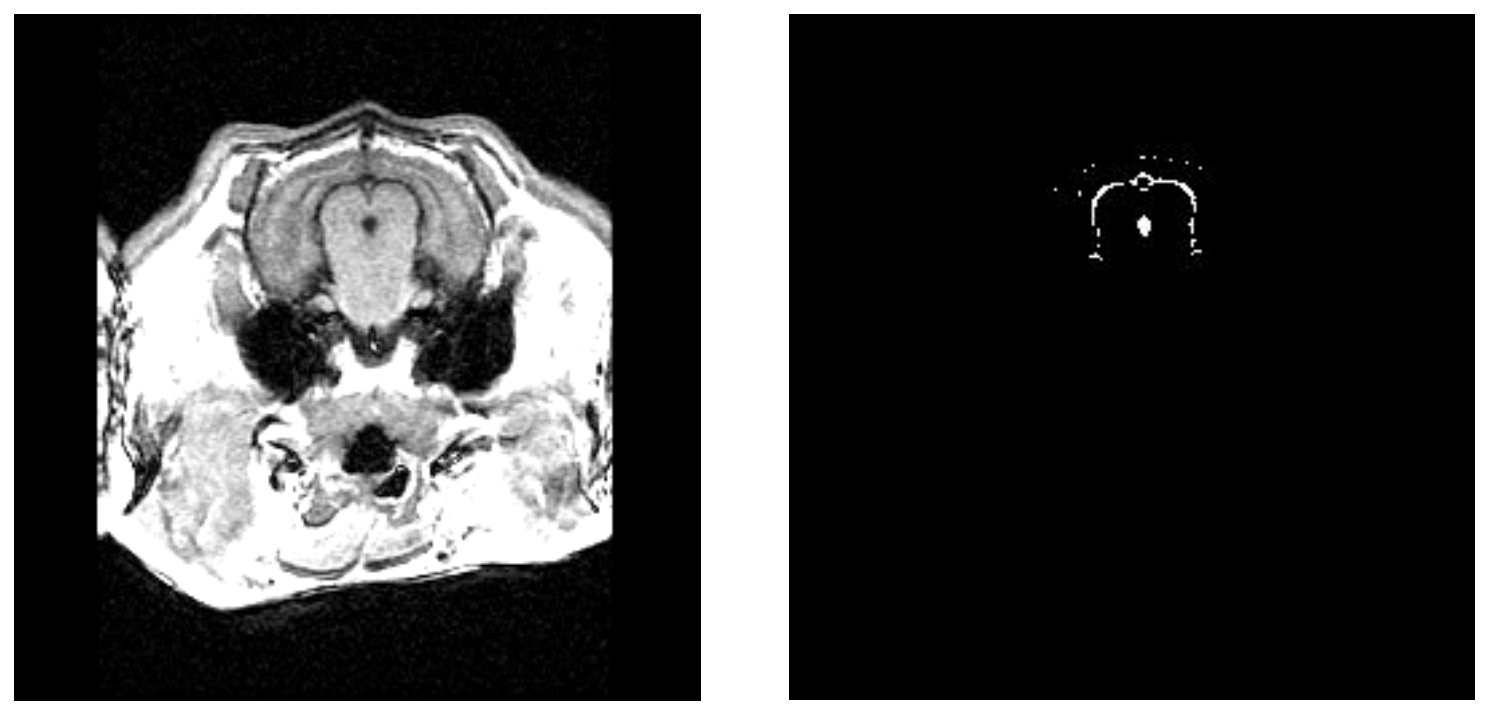

Figure 4.8: Automated seed point selection - D443 FSPGR Image 24 

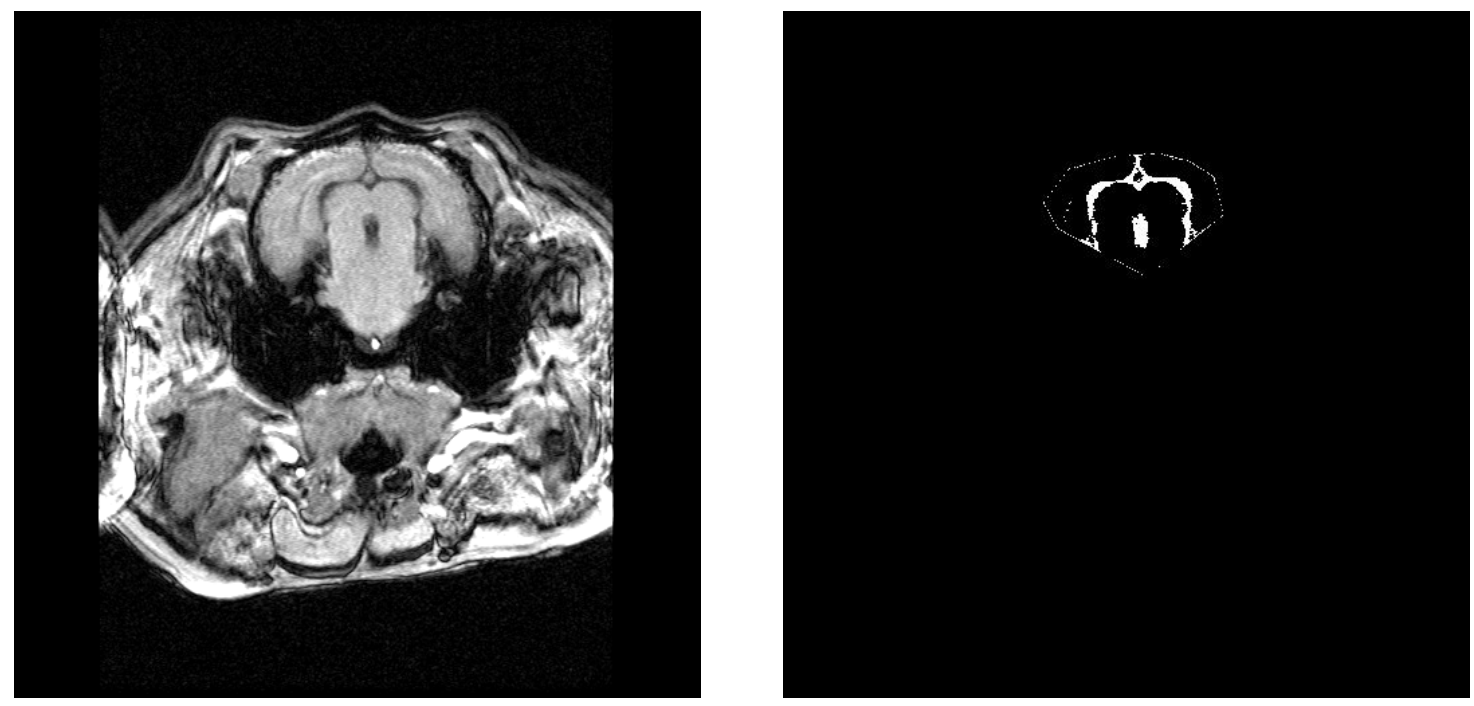

Figure 4.9: Manual seed point selection - D443 TOF Image 27
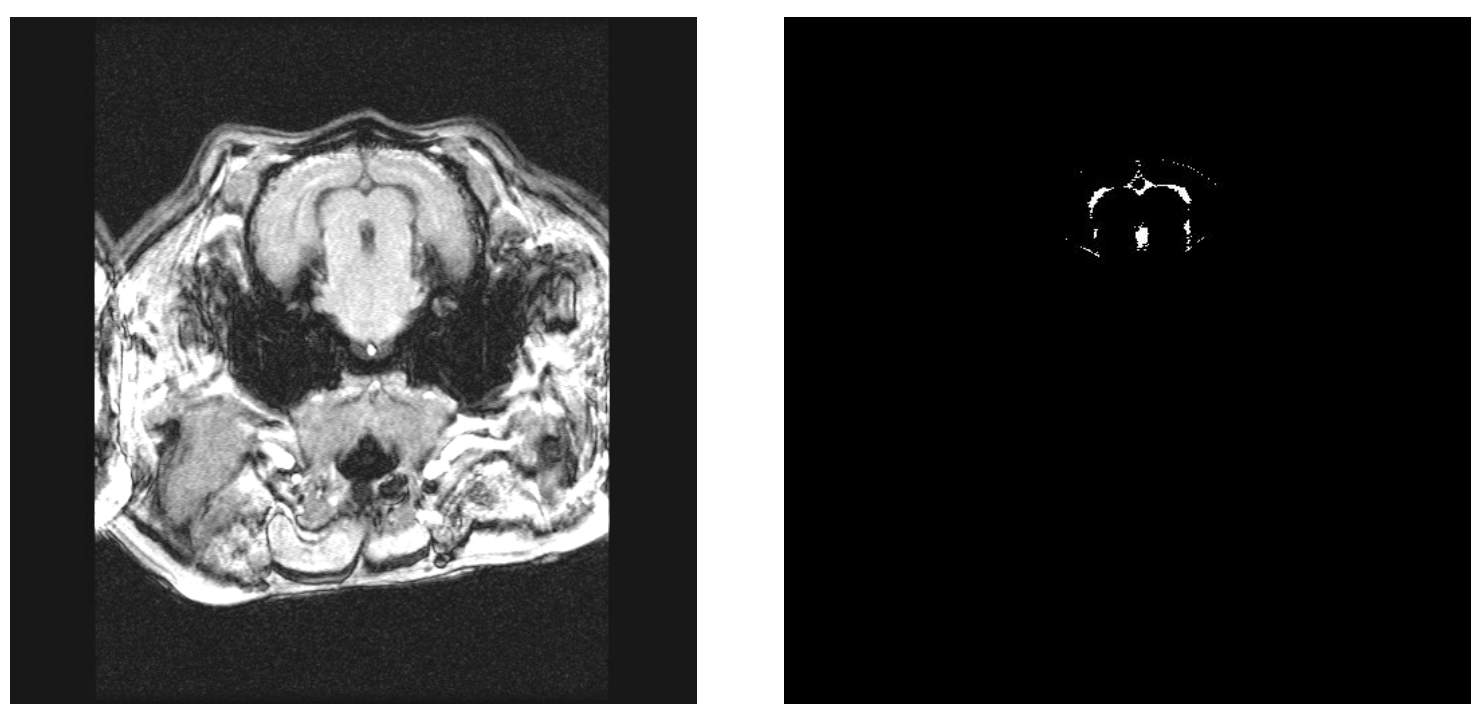

Figure 4.10: Automated seed point selection - D443 TOF Image 27 


\subsubsection{Automated Ventricle Segmentation Process}

The automated ventricle segmentation process was explained in the pervious chapter. It involved two stages: drawing seed point region and subsequently, drawing brain ROI. The results of the ventricle extraction with the entire automated process are shown below.

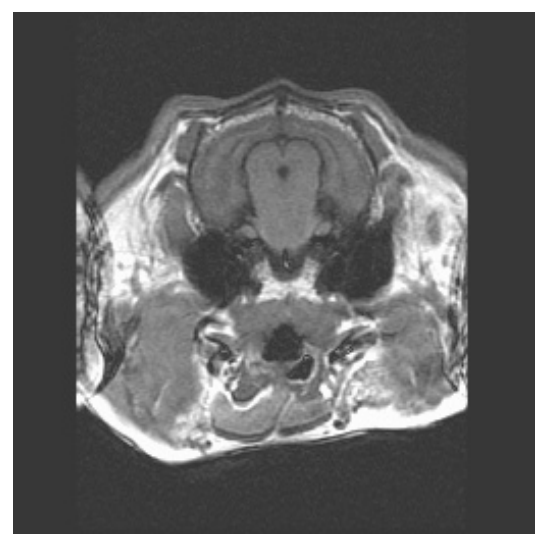

a) D443 FSPGR Image 25

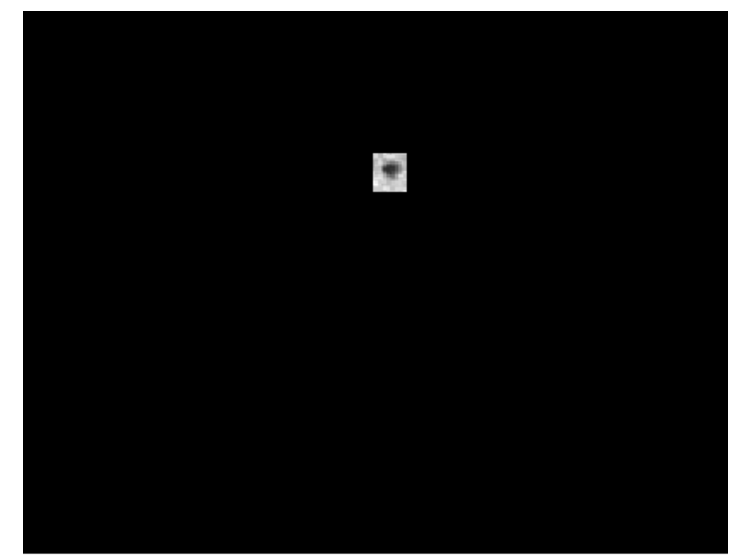

c) Automated seed point region

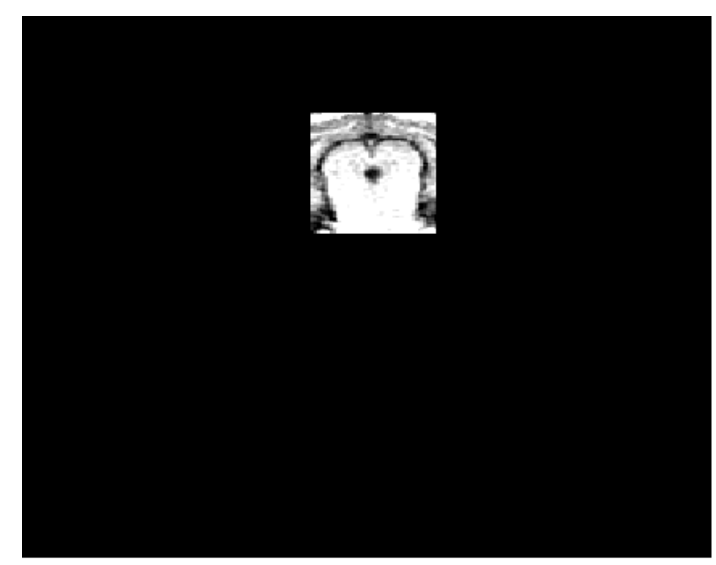

b) Brain ROI with ventricles

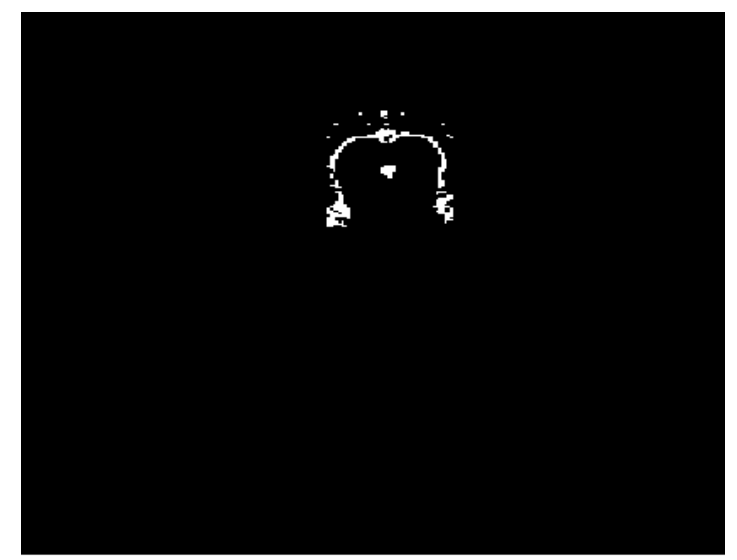

d) Automated ventricle extraction

Figure 4.11: D443 FSPGR Image 25 - Automated ventricle extraction process 


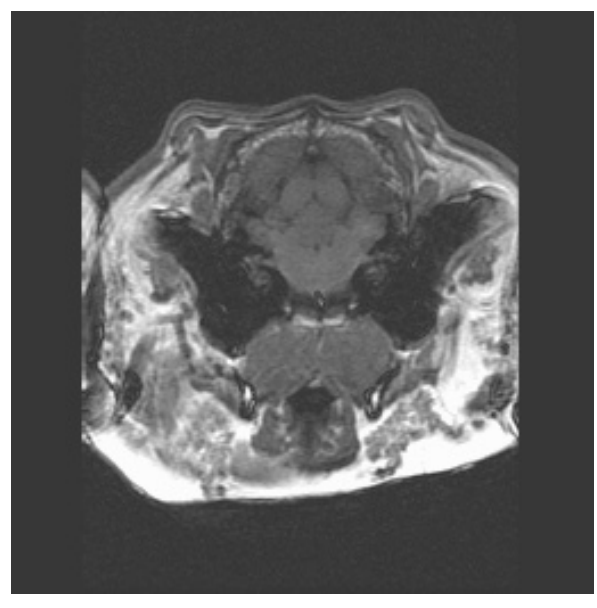

a) D443 FSPGR Image 20

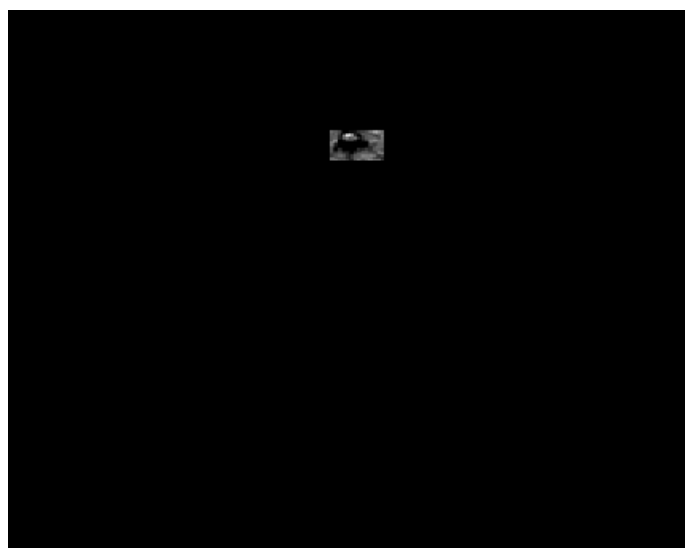

c) Automated seed point region

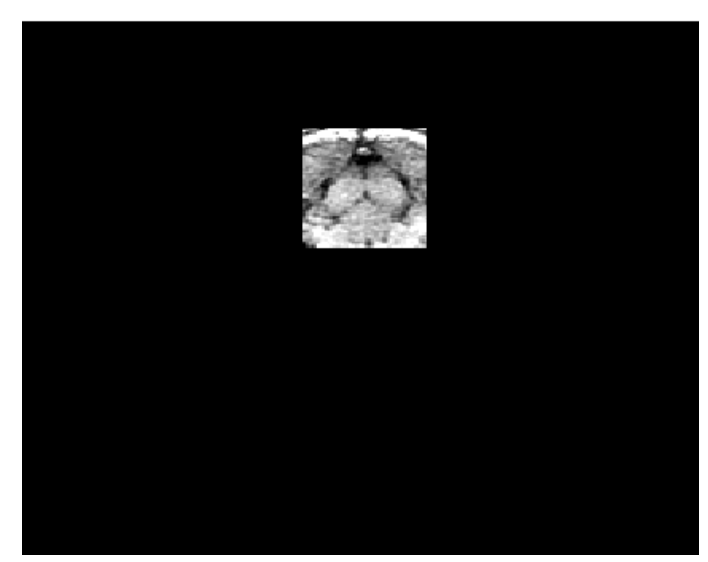

b) Brain ROI with ventricles

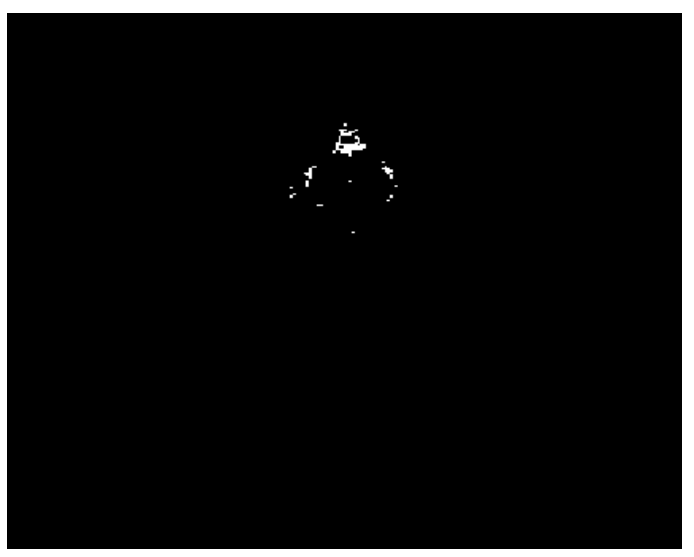

d) Automated ventricle extraction

Figure 4.12: D443 FSPGR Image 20 - Automated ventricle extraction process 


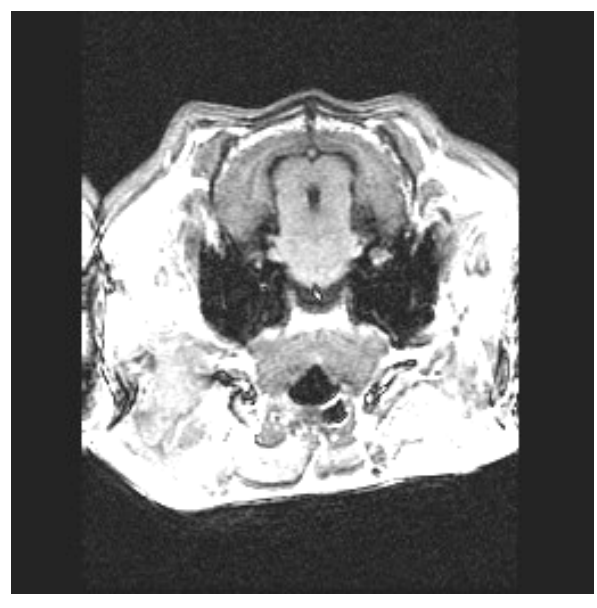

a) D443 FSPGR Image 23

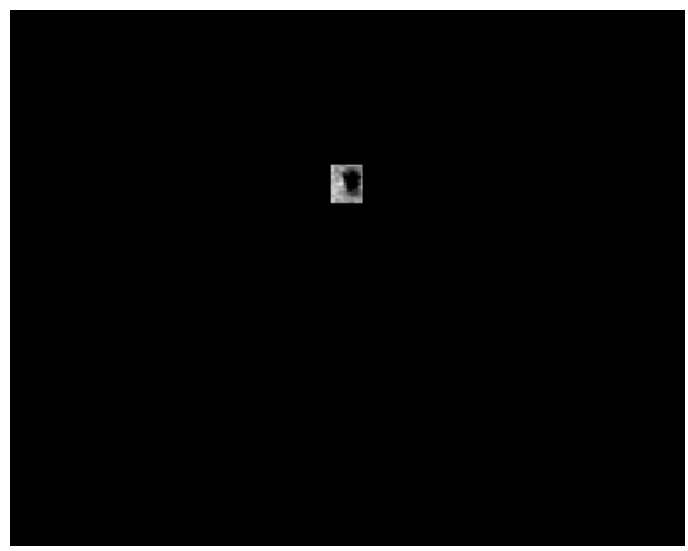

c) Automated seed point region

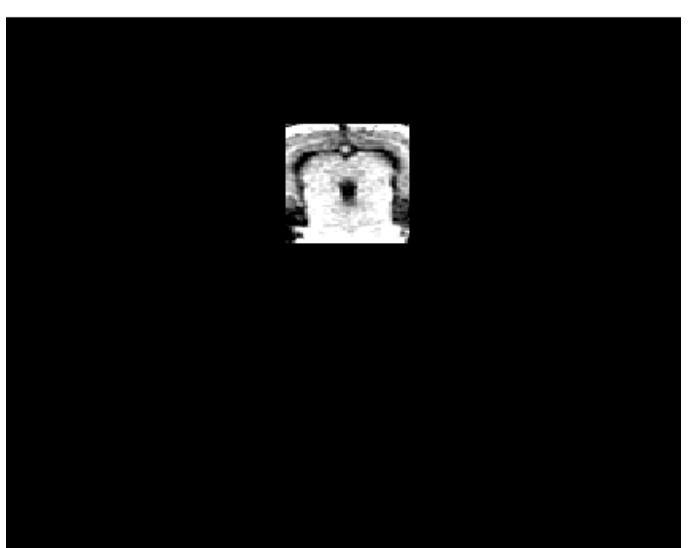

b) Brain ROI with ventricles

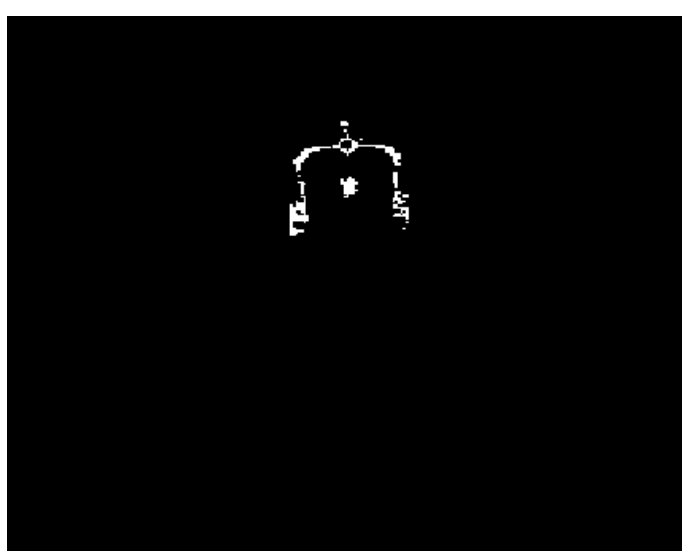

d) Automated ventricle extraction

Figure 4.13: D443 FSPGR Image 23 - Automated ventricle extraction process 


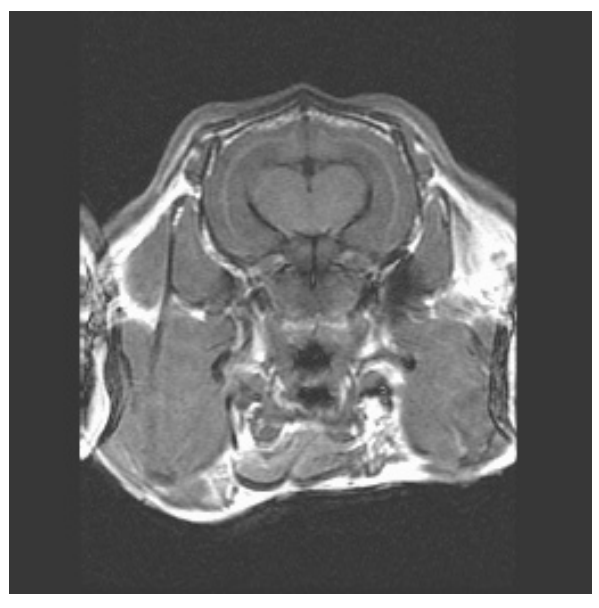

a) D443 FSPGR Image 29

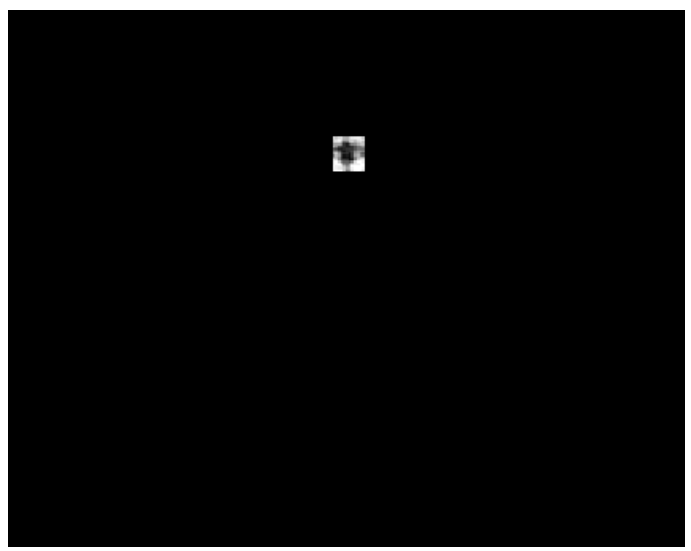

c) Automated seed point region

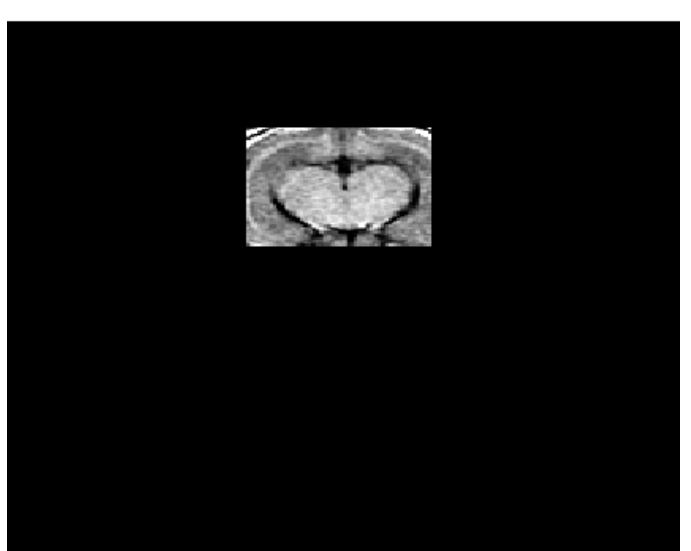

b) Brain ROI with ventricles

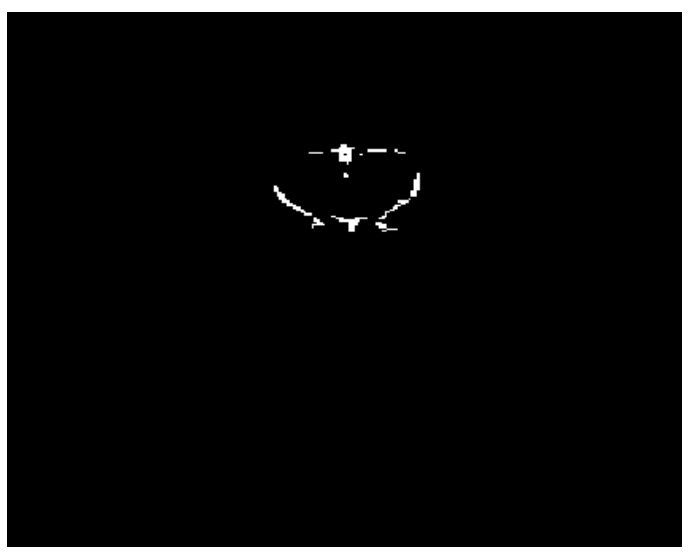

d) Automated ventricle extraction

Figure 4.14: D443 FSPGR Image 29 - Automated ventricle extraction process 


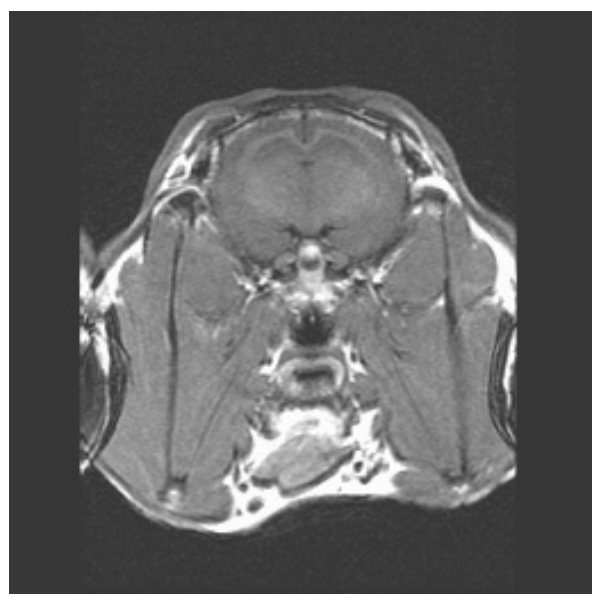

a) D443 FSPGR Image 35

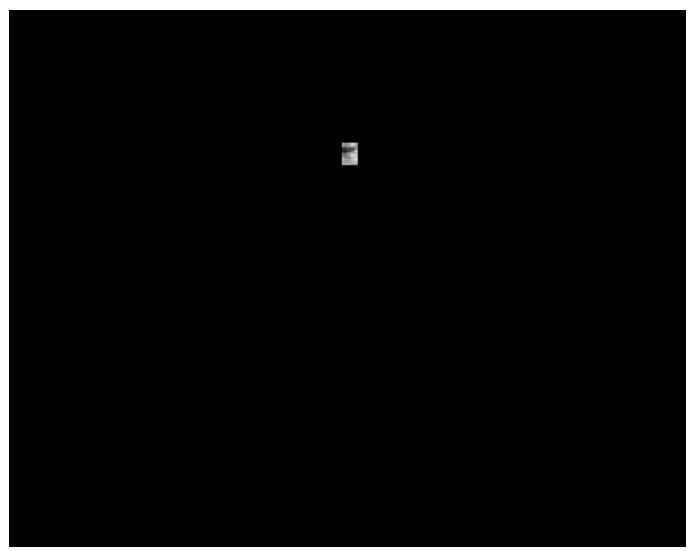

c) Automated seed point region

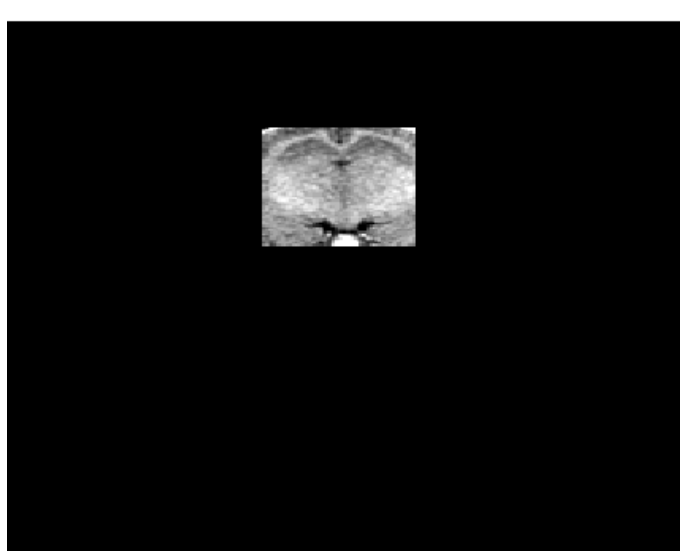

b) Brain ROI with ventricles

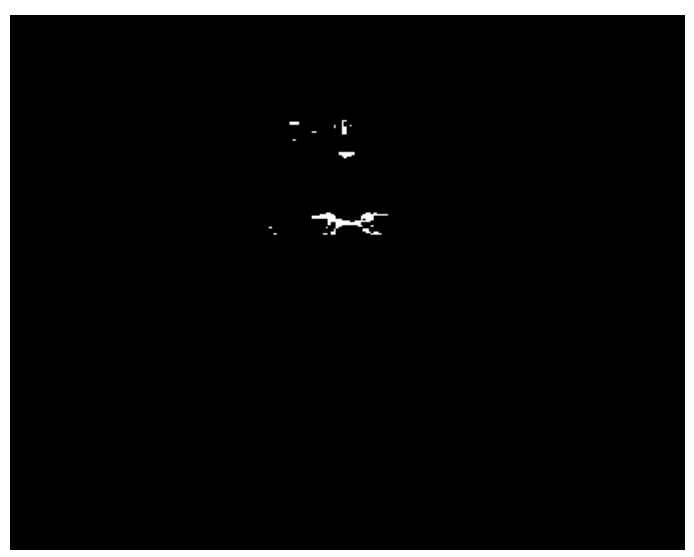

d) Automated ventricle extraction

Figure 4.15: D443 FSPGR Image 35 - Automated ventricle extraction process 
The ventricle volume was obtained by adding the ventricle results from each slice for each animal. The results are shown in Table below.

Table 4-3: Ventricular volume results

\begin{tabular}{|l|l|l|l|}
\hline \multicolumn{2}{|l|}{ Normal Diet + Distilled $\mathrm{H}_{2} \mathrm{O}$} & \multicolumn{2}{l|}{$\begin{array}{l}\text { 12 weeks 2\% Cholesterol Diet + Distilled } \\
\mathrm{H}_{2} \mathrm{O}+12 \mathrm{ppm} \mathrm{Cu}\end{array}$} \\
\hline Animal & FSPGR & Animal & FSPGR \\
\hline D375 & 2354.1 & D397 & 1420.14 \\
D378 & 2743.9 & D455 & 1748.78 \\
D443 & 2422.28 & D459 & 1615.53 \\
\hline
\end{tabular}




\section{CHAPTER 5: DISCUSSION AND CONCLUSIONS}

In this thesis, Gabor wavelets was applied to brain ROI drawn from whole head rabbit MRI (FSPGR) for segmenting ventricles from two groups of animals: normal rabbits and cholesterol-fed animals.

The results from Table 4.3 indicate that the volume of the ventricles in the normal rabbit brain is higher than the ventricular volume of the cholesterol-fed rabbit brain. This was not the predicted results. In our study, the ventricles were extracted from the brain ROI determined by the automated process. It can be observed from the results shown in the chapter 4 , that other non-ventricular structures were also included in the ventricular volume measurements. The different amount of structures with an anatomatical ventral plane of the brain was included as a part of ventricles. Hence, it is necessary to re-define the process of automating the brain ROI so that the ventricles only can be extracted.

The performance of this algorithm can be analyzed using a synthetic image as shown in Figure 5.1. The synthetic image was created manually resembling a portion of the rabbit brain MRI from the skull on the top to the region just below the third ventricles.

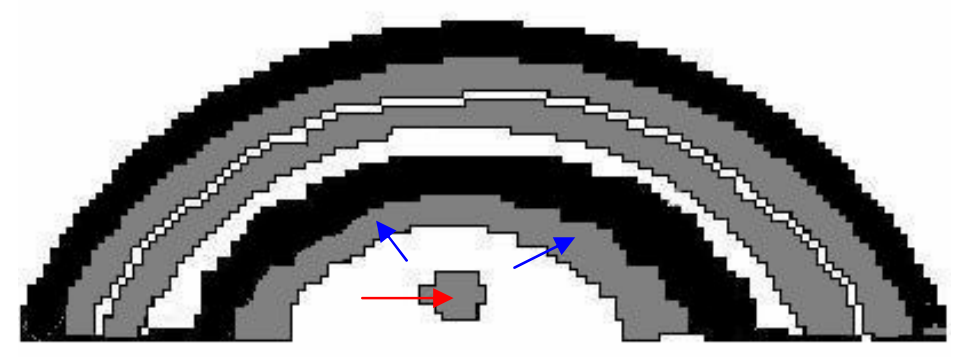

Figure 5.1: Synthetic image

Lateral ventricle (blue) and third ventricle (red)

The ventricular regions are known in the synthetic image. The lateral and third ventricle indicated in the Figure needs to be segmented to measure the volume. The grey 
level of the ventricles of synthetic image should be as close as the grey level of the ventricles of the rabbit brain MRI. Then the filtering and threshold operation can be performed on the synthetic image. The error can be calculated using the prior information and the extracted ventricles. Thus the efficiency of this algorithm can be obtained.

An understanding of the Gabor wavelets is required before applying the algorithm to the MR data sets. Important parameters like the number of scales and orientations, upper frequency, lower frequency and window size need to be considered. Moreover, as the number of filters and window size increases, the Gabor wavelets algorithm calculation times also increases. An important future step for this project is the minimization of processing time by translating the MATLAB code into $\mathrm{C}++$ and reevaluating the algorithm. That is a large project that could take a significant amount of time.

In spite of the intuitive sense that adding TOF images would improve the ventricle extraction, our results indicate that including TOF to the feature vectors did not yield better results. This is properly because the TOF images have lower contrast than the FSPGR images.

Anders Dale et. al (2005) performed automated segmentation of neuroanatomic structures in multispectral MR microscopy of mouse brain. Apriori knowledge was incorporated in their experiment for segmentation purpose. It required manual interference to draw the neuroanatomic structures for training data. Our work differs the method is operator independent and we were able to segment ventricles within the brain ROI selected by automated process.

Another group used Gabor wavelets on histological rat brain images (ref) for registration purposes. Our motivation was for a different application. To the best of our knowledge, our work is the first automated ventricle segmentation using Gabor wavelets on rabbit brain extracted from whole head rabbit MRI. The Gabor wavelet technique separated the ventricles from the rest of the rabbit brain tissues. 
In summary and conclusion, the Gabor wavelets were used for ventricular measurement in rabbit brain. In this work, the input image for the ventricle segmentation process was a brain ROI including the ventricles rather than the brain. From the experimental results, it can be seen that the results can be improved if the brain was segmented from the whole head rabbit MRI. We were unable to use few of the image datasets has the images had turned out a little as seen in Figure 5.2. As a result, the peak on the skull could not be detected. Future work needs to be done to realign (or rotate) the images so that the peak will be positioned along the midline of the brain. Ventricular measurements would be easy if the animal scans are performed or images are acquired according to the requirement of the study. Alternately, post-processing algorithms such as AIR (ref) can be used to rotate or re-align the acquired images.

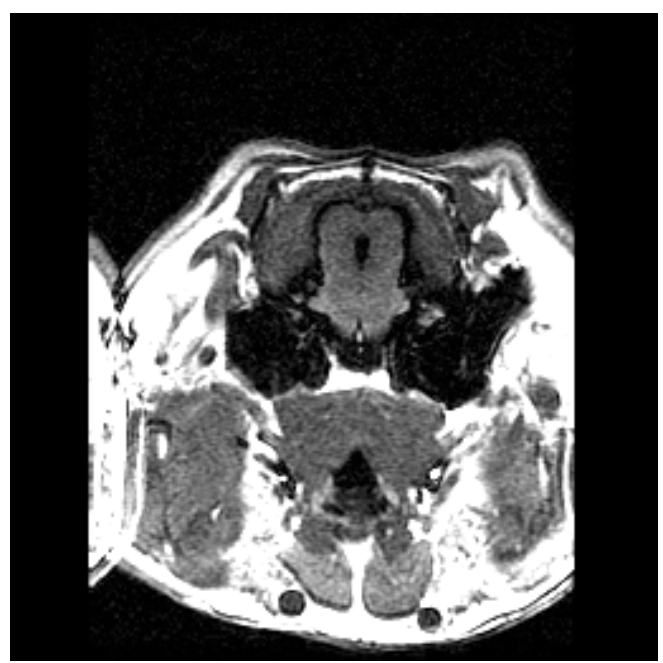

a) Animal D377 image

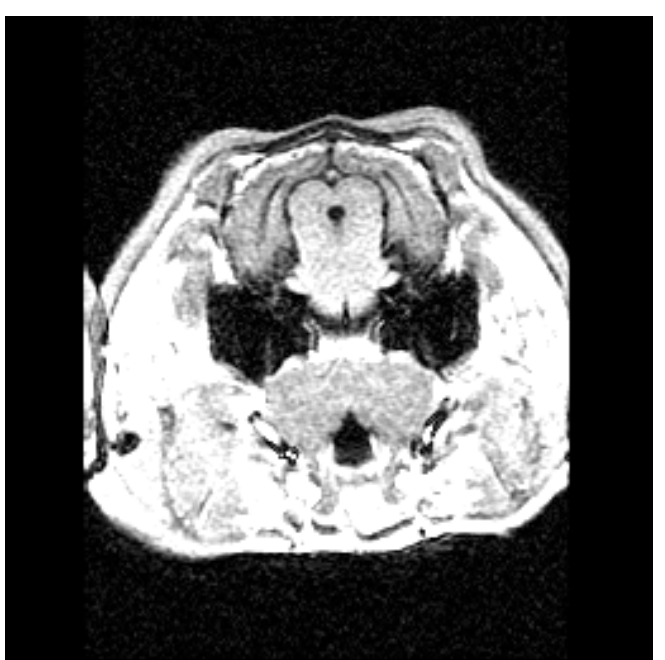

b) Animal D444 image

Figure 5.2: Rotated images 


\section{BIBLIOGRAPHY}

1. R. Sigal, D. Doyon, Ph. Halimi, H. Atlan, Magnetic resonance imaging basis for interpretation, New York: Springer-Verlag, December 1997.

2. P. Sprawls, Magnetic resonance imaging: principles, methods and techniques, Medical Physics Publishing, 2000.

3. T.F. Cootes, A. Hill, C.J. Taylor, J. Haslam, 1994. The use of active shape models for locating structures in medical images. Image and Vision Computing, 12 (6): 355-366.

4. C. DeCarli, J.V. Haxby, J.A. Gillette, 1992. Longitudinal changes in lateral ventricular volume in patients with dementia of the Alzheimer type. Neurology $42,2029-2036$.

5. D.G. Murphy, C.D. DeCarli, E. Daly et. Al., 1993. Volumetric magnetic resonance imaging in men with dementia of the Alzheimer type: correlations with disease severity. Biol. Psychiatry 34: 612-621.

6. R. Sigal, Magnetic resonance imaging: basis for interpretation, New York: Springer-Verlag, 1998.

7. J. Yang, J.S. Duncan, 2004. 3D image segmentation of deformable objects with joint shape-intensity prior models using level sets. Medical Image Analysis, 8:285-294.

8. J. Yang, L.H. Staib, J.S. Duncan, 2004. Neighbor-constrained segmentation with level set based 3-D deformable models. IEEE Transactions on Medical Imaging, 23 (8): 940-948.

9. L. Ferrarini, W. M. Palm, H. Olofsen, M. A. van Buchem, J.H.C. Reiber, F.Admiraal-Behloul, 2006. Shape differences of the brain ventricles in Alzheimer's disease. NeuroImage 32: 1060-1069.

10. URL: http://alz.org/alzheimers_disease_what_is_alzheimers.asp (date retrieved: November 2007).

11. URL: http://www.alz.org/brain/10.asp (date retrieved: November 2007).

12. URL: http://www.mr-tip.com (date retrieved: November 2007).

13. URL: http://www.med.nagasaki-u.ac.jp/radiology (date retrieved: November 2007). 
14. C. Liu, May 2004. Gabor-based kernel PCA with fractional power polynomial models for face recognition. IEEE Trans. on Patt. Anal. and Machine Intell., 26 (5): $572-581$.

15. C. Liu and H. Welscher, 2000. Independent component analysis of Gabor features for face recognition. IEEE Trans. Neural Networks, 14 (4): 919-928.

16. N.C. Anderasen, V.W. Swayze, M. Flaum, W.R. Yates, S. Arndt, C. McChesney, 1990. Ventricular enlargement in schizophrenia evaluated with computed tomographic scanning effects: effects of gender, age, and stage of illness,. Arch Gen Psychiatry 47: 1008-1015.

17. M.E. Shenton, R. Kikinis, R.W. McCarley, D. Metcalf, J. Tieman, F.A. Jolez 1991. Application of automated MRI volumetric measurement techniques to the ventricular system in schizophrenics and normal controls. Schizophr Res 5: 103113.

18. G. Chetelat and Jean-Claude Baron, 2003. Early diagnosis of Alzheimer's Disease: contribution of structural neuroimaging: NeuroImage, 18: 525-541

19. G.B. Frisoni, September 2001. Structural imaging in the clinical diagnosis of alzheimer's disease: problems and tools. Journals of Neurology, Neurosurgery, and Psychiatry, 70(6): 711-718.

20. L. Chen, D. Bouley, B. Harris, K. Butts, 2000. Quantitative comparison of MR imaged focused ultrasound lesions and cell death zones in the in vivo rabbit brain, Proceedings of the $22^{\text {nd }}$ Annual EMBS International Conference, July 23-28, Chicago IL.

21. D.L. Sparks, and B.G. Schreurs 2003. Trace amounts of copper in water induce \{beta\}-amyloid plaques and learning deficits in a rabbit model of Alzheimer's disease 100; 11065-11069; PNAS.

22. M. Tuceryan, A.K. Jain. Texture analysis, Handbook of Pattern Recognition \& Computer Vision, chapter 2.1, World Scientific, Singapore, 1993.

23. R.M. Haralick, K. Shanmugam, and I. Dinstein, June 1973. Textural features for image classification, IEEE Trans. Syst. Man. Cybern., vol. SMC-3, no. 6, pp. 610621. 
24. D.A. Clausi and M.E. Jernigan, 2000. Designing Gabor filters for optimal texture separability, Pattern Recognition., vol. 33, pp. 1835-1849.

25. A. Kumar and G. K. H. Pang, 2002. Defect detection in texture materials using Gabor filters, IEEE Trans. Ind. Applicat., vol. 38, no. 2, pp. 425-440.

26. P. Rivaz and N. Kingsbury, September 2000. Fast segmentation using level set curves of complex wavelet surfaces, in Proc. IEEE Int. Conf. on Image Processing, Vancouver, BC, Canada, pp. 592-595.

27. A. Ahmadian, A. Mostafa, M.D. Abolhassani, Y. Salimpour, A texture classification method for diffused liver diseases using Gabor wavelets, Proceedings of the 2005 IEEE, Engineering in Medicine and Biology $27^{\text {th }}$ Annual Conference, Shanghai, China, Sept. 1-4, 2005.

28. Chien-Cheng Lee, Sz-Han Chen, Hong-Mind Tsai, Pau-Choo Chung, and YuChun Chiang, Discrimination of liver diseases from CT Images based on Gabor filters, Proceedings of the $19^{\text {th }}$ IEEE Symposium on Computer-Based Medical Systems (CBMS'06), Page(s):203 - 206 , Digital Object Identifier 10.1109/CBMS.2006.77

29. R.J. Ferrari, R.M. Rangayyan, J.E.L. Desautels, and A.F. Frere, September 2001. Analysis of asymmetry of mammograms via directional filtering with Gabor wavelets, IEEE Trans. on Medical Imaging, Vol. 20, No. 9.

30. S. Udomhusakul, Edge detection in ultrasonic images using Gabor filters, IEEE Region 10 Conference, Volume A, 21-24 Nov. 2004 Page(s):175-178,Vol.1 Digital Object Identifier 10.1109/TENCON.2004.1414385.

31. B.S. Manjunath and W.Y. Ma, Aug. 1996. Texture features for browsing and retrieval of image data, IEEE Trans. Pattern Analysis and Machine Intelligence, Vol 18, No. 8, pp. 837 - 842, Aug. 1996.

32. B.S. Manjunath, C. Shekhar, and R. Chellappa, Apr. 1996. A new approach to image feature detection with applications, Pattern Recognition, Apr. 1996.

33. J. Xie, Y. Jiang, and Hung-tat Tsui, Jan. 2005. Segmentation of kidney from ultrasound images based on texture and shape priors, IEEE. Trans. Medical Imaging, vol. 24, no. 1, pp. 45-57. 
34. A.C. Bovik, M. Clark, and W.S. Geisler, Jan. 1990. Multichannel texture analysis using localized spatial filters, IEEE Trans. Pattern Analysis and Machine Intelligence, vol.12, no.1, pp.55-73.

35. R.C. Gonzalez and R.E. Woods, Digital Image Processing, $2^{\text {nd }}$ Edition, Prentice Hall, 2002.

36. M.I. Miller, G.E. Christensen, Y. Amit, and U. Grenander, 1993. Mathematical textbook of deformable neuroanatomies, In Proceedings of National Academy of Science U.S.A, volume 90, pages 11944-11948.

37. K. Okada, M.J. Lyons, On Gabor Wavelet-based Image Processing for NisslStained Rat Brain Slices.

38. J. G. Daugman, 1998. Complete discrete 2-D Gabor transforms by neural networks for image analysis and compression," IEEE Transactions on Acoustics, Speech, and Signal Processing, 36:1169-1179.

39. L. W. Swanson. Brain maps: Structure of the Rat Brain, Elsevier Science Publishers, Netherlands, 1992.

40. M. Turner, 1986. Texture discrimination by Gabor functions, Biol. Cybern., vol. 55, pp. 71-82.

41. I. Fogel and D. Sagi, 1989. Gabor filters as texture discriminator, Biol. Cybern., vol. 61, pp. 103-113.

42. M. Clark and A. Bovik, June 1989. Experiments in segmenting texton patterns using localized spatial filters, Pattern Recogn., vol. 22, pp. 707-717.

43. A.A. Ali, A.M. Dale. A. Badea, and G.A. Johnson, 2005. Automated segmentation of neuroanatomical structures in multispectral MR microscopy of the mouse brain, NeuroImage 27, pp. 425-435.

44. A.K. Jain, F. Farrokhnia, Dec. 1991. Unsupervised texture segmentation using Gabor filters, Pattern Recognition, vol.23, no.12, pp.1167 - 1186.

45. D. Gabor, 1946. Theory of Communication, J. Inst. Elec. Eng. (London), vol. 93, pp. $429-457$.

46. J. Daugman, July 1985. Uncertainty relation for resolution in space, spatial frequency and orientation optimized by two-dimensional visual cortical filters, J. Opt. Soc. Amer. A, vol. 2, pp. 1160 - 1169. 
47. D. Dunn, W. Higgins, and J. Wakeley, Mar. 1992. 2-D analysis of Gabor filters output signatures for texture segmentation, in Proc. IEEE Int. Conf. Acoust., Speech Signal Processing, pp. 65-68, vol. 3.

48. D. Dunn, W. Higgins, and J. Wakeley, Feb. 1994. Texture segmentation using 2D Gabor elementary functions, IEEE Trans. Pattern Anal. Mach. Intell., vol. 16, no. 2 , pp. 130-149.

49. D. Dunn and W. Higgins, Apr. 1993. Optimal Gabor-filter design for texture segmentation, IEEE Int. Conf. Acoustic., Speech Signal Processing, Minneapolis, MN, pp. 37-40, vol. 5.

50. D. Dunn and W. Higgins, July 1995. Optimal Gabor filters for texture segmentation, IEEE Trans. on Image Processing, vol. 4, no. 7, pp. 947 - 964.

51. H. Rusinek, M.J. de Leon, A.E. George, et al., 1991. Alzheimer disease: measuring loss of cerebral gray matter with MR imaging. Radiology, 178:109114.

52. A.E. George, M.J. de Leon, S. Rosenbloom, et al., 1983. Ventricular volume and cognitive deficit: a computed tomographic study. Radiology, 149: 493-498.

53. M.J. de Leon, A.E. George, B. Reisberg, et al., 1989. Alzheimer's disease: longitudinal CT studies of ventricular change. AJNR: 10: 371-376.

54. H.G. Schnack, H.E. Hulshoff Pol, W.F.C. Baare, M.A. Viergever and R.S. Kahn, 2001. Automatic segmentation of the ventricular system from MR images of the human brain, NeuroImage 14, 95-104.

55. M.I. Kohn, N.K. Tanna, G.T. Herman, S.M. Resnick, P.D. Mozley, R.E. Gur, A. Alavi, R.A. Zimmerman, R.C. Gur. 1991. Analysis of brain and cerebrospinal fluid volumes with MR Imaging. Part1. Methods, reliability, and validation. Radiology178:115-122.

56. M.E. Brandt, T.P. Bohan, L.A. Kramer, J.M. Fletcher, 1994. Estimation of CSF, white and gray matter volumes in hydrocephalic children using fuzzy clustering of MR images. Computerized Med. Imaging Graphics 18:25-34.

57. N. Saeed, B.K. Puri, A. Oatridge, J.V. Hajnal, and I.R. Young, 1998. Two methods for semi-automated quantification of changes in ventricular volume and 
their use in schizophrenia. Magnetic Resonance Imaging, Vol.16, No.10, pp.12371247.

58. A.J. Worth, N. Makris, M. R. Patti, J. M. Goodman, E. A. Hoge, V. S. Caviness, Jr. and D. N. Kennedy, April 1998. Precise segmentation of the lateral ventricles and caudate nucleus in MR brain images using anatomically driven histograms. IEEE Trans. on Medical Imaging, vol. 17 (2).

59. D. A. Clausi, Mar. 2001. Comparison and fusion of co-occurrence, Gabor and MRF texture features for classification of SAR sea-ice imagery, AtmosphereOcean, vol.39, is.3, pp.183-194.

60. D.A Clausi, and M.E. Jernigan, 1998. A fast method to determine co-occurrence texture features, IEEE Transactions on Geosciences and Remote Sensing, Vol. 36, No. 1, pp. $298-300$.

61. T. Saito, H. Kudo and S. Suzuki, Oct. 1996. Texture image segmentation by optimal Gabor filters, Signal Processing, 1996, 3rd International Conference, vol. 1, no. 14-18, Oct 1996, pp. $380-383$.

62. D.A Clausi, and B. Yue, 2004. Comparing co-occurrence probabilities and Markov random fields for texture analysis, IEEE Transactions on Geosciences and Remote Sensing, Vol. 42, No. 1, pp. 215-228.

63. URL: http://afni.nimh.nih.gov/afni/ (date retrieved: November 2007).

64. URL: http://serendip.brynmawr.edu/bb/kinser/Size1.html (date retrieved: November 2007).

65. URL: http://www.techfak.uni-bielefeld.de/ags/ni/projects/percgroup/ (date retrieved: November 2007).

66. URL: http://www.learnthat.com/define/view.asp?id=7095 (date retrieved: November 2007). 